University of Rhode Island

DigitalCommons@URI

Open Access Master's Theses

2020

\title{
CHARACTERIZATION OF DROP-STITCH CONSTITUENT MATERIALS AND INFLATED PANEL RESPONSE
}

Ryan A. Buglio

University of Rhode Island, ryan_buglio@my.uri.edu

Follow this and additional works at: https://digitalcommons.uri.edu/theses

\section{Recommended Citation}

Buglio, Ryan A., "CHARACTERIZATION OF DROP-STITCH CONSTITUENT MATERIALS AND INFLATED PANEL RESPONSE" (2020). Open Access Master's Theses. Paper 1856.

https://digitalcommons.uri.edu/theses/1856

This Thesis is brought to you for free and open access by DigitalCommons@URI. It has been accepted for inclusion in Open Access Master's Theses by an authorized administrator of DigitalCommons@URI. For more information, please contact digitalcommons-group@uri.edu. 
CHARACTERIZATION OF DROP-STITCH CONSTITUENT MATERIALS AND INFLATED PANEL RESPONSE

BY

RYAN A. BUGLIO

A THESIS SUBMITTED IN PARTIAL FULFILLMENT OF THE

REQUIREMENTS FOR THE DEGREE OF

MASTER OF SCIENCE

IN

MECHANICAL ENGINEERING

UNIVERSITY OF RHODE ISLAND

2020 
MASTER OF SCIENCE THESIS

$\mathrm{OF}$

RYAN A. BUGLIO

\title{
APPROVED:
}

Thesis Committee:

\author{
Major Professor David G. Taggart \\ Chengzhi Yuan \\ George Tsiatas \\ Nasser H. Zawia \\ DEAN OF THE GRADUATE SCHOOL
}

\section{UNIVERSITY OF RHODE ISLAND}




\begin{abstract}
Drop-stitch inflatable structures are of increasing significance in aerospace, naval, and military applications. The advantages of drop- stitch inflatable structures are high strength to weight ratios, rapid deployment capabilities and ease of storage. The stiffness of the structure increases with inflation pressure and is dependent on the material properties of the panel skin.

The objective of this research was to characterize the constituent material properties and mechanical response of drop-stitch inflatable panels subjected to various load conditions. This research aimed to characterize the nonlinear elastic response of drop-stitch inflatable panels by using a new material system in comparison to previous studies. These characterizations included three dimensional digital image correlation, uniaxial and biaxial loading, and panel inflation and bending experiments.

Material properties of the drop-stitch inflatable panel skin were experimentally determined across multiple experiments utilizing three dimensional-digital image correlation. In addition, three dimensional digital image correlation was used to characterize panel skin surface displacements and strains in a way not done before in previous drop-stitch structure research.

The results of this research found that relevant material properties of the dropstitch inflatable panel were able to be experimentally determined through multiple different experiments, however these material property values are extremely sensitive to load conditions. Further research is required to accurately predict the structural behavior from constituent characterization data.
\end{abstract}




\section{ACKNOWLEDGMENTS}

First, I would like to acknowledge and thank Dr. David Taggart for all of his

help and guidance throughout my thesis research. I greatly appreciate not only the academic support and advice but also the personal trust he put in me assisting in his work over the past year.

I would also like to acknowledge and thank my friend and fellow graduate student Dylan Stephanik for his contributions and assistance to this research.

In addition, I'd like to acknowledge and thank Chris Hart and Milo Ferrazzoli from Navatek LLC for their contributions to this research.

Furthermore, I'd like to acknowledge and thank Professor Tsiatas for allowing me to use the Civil Engineering structures laboratory to conduct some of the testing for this research.

Lastly, I'd like to thank my friends and family; my parents Deborah and Frank, and my brother Matthew for their support and encouragement throughout my entire academic career. 


\section{TABLE OF CONTENTS}

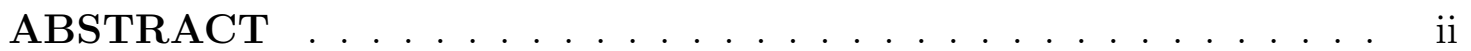

ACKNOWLEDGMENTS ................. . iii

TABLE OF CONTENTS $\ldots \ldots \ldots \ldots \ldots$ iv

LIST OF FIGURES $\ldots \ldots \ldots \ldots \ldots \ldots \ldots$ vi

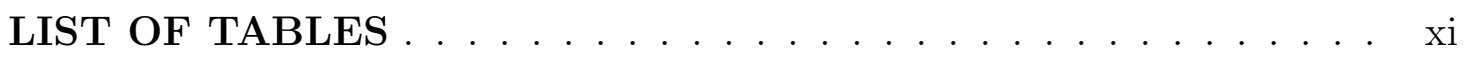

\section{CHAPTER}

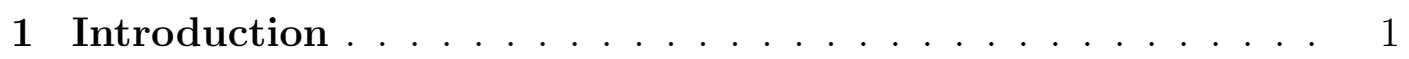

1.1 Drop-stitch inflatable structures . . . . . . . . . . . . 1

1.1.1 Drop-stitch inflatable structure applications . . . . . . 3

1.2 Motivation ....................... . . 4

1.3 Objective of research $\ldots \ldots \ldots \ldots \ldots \ldots$

1.4 Thesis overview $\ldots \ldots \ldots \ldots \ldots \ldots$

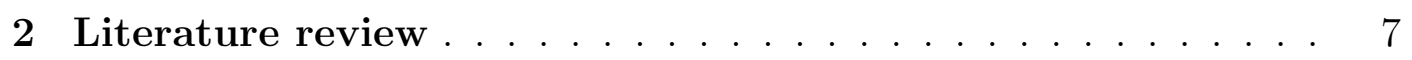

2.1 Inflatable structures $\ldots \ldots \ldots \ldots \ldots \ldots \ldots$

2.2 Inflatable panel beam models $\ldots \ldots \ldots \ldots$

3 Methodology ....................... 11

3.1 Theory . . . . . . . . . . . . . . . . . . 11

3.1.1 Digital image correlation . . . . . . . . . . . . 11

3.1.2 Mechanics of laminated composites . . . . . . . . 13

3.1 .3 Inflation stress . . . . . . . . . . . . . . . . 18 


\section{Page}

3.1.4 Four-point bending . . . . . . . . . . . . . . . 20

3.2 Experimental preparation and setup . . . . . . . . . . . 24

3.2.1 Drop-stitch inflatable panel build . . . . . . . . . . . 24

3.2 .2 Digital image correlation . . . . . . . . . . . . . 28

3.2 .3 Uniaxial tensile testing . . . . . . . . . . . . . . . 33

3.2 .4 Biaxial tensile testing . . . . . . . . . . . . . . 36

3.2 .5 Inflation testing . . . . . . . . . . . . . 38

3.2 .6 Four-point bend testing . . . . . . . . . . . . . . 40

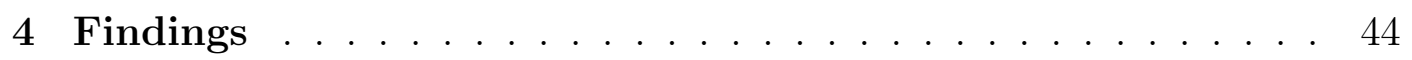

4.1 Uniaxial tensile testing . . . . . . . . . . . . . . . . 44

4.2 Biaxial tensile testing . . . . . . . . . . . . . . 51

4.3 Inflation testing $\ldots \ldots \ldots \ldots \ldots \ldots \ldots$

4.4 Four-point bend testing . . . . . . . . . . . . . . 62

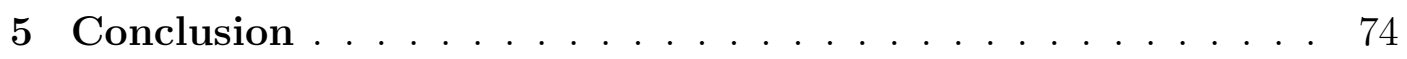

5.1 Conclusions . . . . . . . . . . . . . . . . . 74

5.2 Recommendations for future research $\ldots \ldots \ldots$. . . . . 76

LIST OF REFERENCES . . . . . . . . . . . . . . . . . 77 APPENDIX

A MATLAB script: Inflation stresses . . . . . . . . . 79

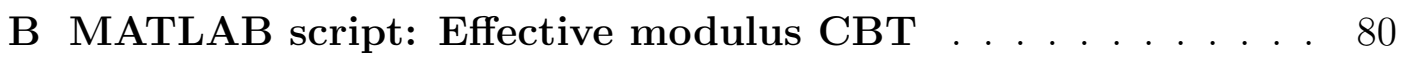

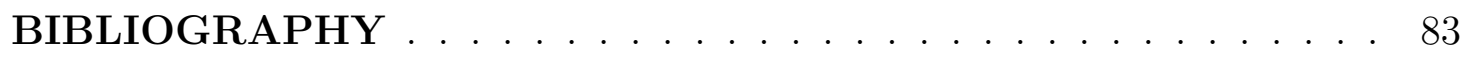




\section{LIST OF FIGURES}

$\begin{array}{ll}\text { Figure } & \text { Page }\end{array}$

1 Drop-stitch skin layers configuration [1] . . . . . . . . . . 2

2 Warp (axial) and weft (transverse) directions [2] . . . . . . . 2

3 Navatek Inflatable Boat Ramp [3] . . . . . . . . . . . . . 3

4 Sierra Nevada Corporation Inflatable Space Habitat [4] . . . . . 3

$5 \quad$ Finite element model of plain-woven air beam four-point bend test $[5] \ldots \ldots \ldots$................... . . . . 8

$6 \quad$ Finite element model of drop-stitch inflatable panel four-point bend test $[6] \ldots \ldots \ldots 10$

$7 \quad$ Digital image correlation surface pattern [7] . . . . . . . . . . . 11

8 Surface pattern DIC software recognition [7] . . . . . . . . . 12

$9 \quad$ Deformed pattern DIC software recognition [7] . . . . . . . . 12

10 Three dimensional digital image correlation using two cameras [7] 13

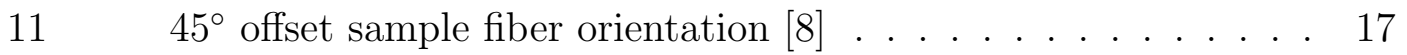

12 Axial and transverse cross-sectional areas . . . . . . . . . . . 19

13 Four-point bending shear and moment diagrams . . . . . . . . . 21

14 Four-point bending panel stress diagram . . . . . . . . . . . . 21

15 Panel cross section geometry simplification . . . . . . . . . 23

16 Drop-stitch inflatable panel . . . . . . . . . . . . . 24

17 Drop-stitch inflatable panel skin layers . . . . . . . . . . . 25

18 Drop-stitch panel fabric . . . . . . . . . . . 26

19 Halkey-Roberts valve . . . . . . . . . . . . . 27

20 Halkey-Roberts valve adapter . . . . . . . . . . . . 27 
21 Panel four-point bend test white base coat . . . . . . . . . . 28

22 Panel four-point bend test full speckled section of panel . . . . . 29

23 Panel four-point bend test speckle pattern . . . . . . . . . . . 30

24 Biaxial and uniaxial tensile test speckle patterns . . . . . . . 31

25 Inflation test speckle pattern . . . . . . . . . . . . . 31

26 Three-dimensional DIC calibration board . . . . . . . . . . . . 32

27 Three-dimensional DIC calibration cross . . . . . . . . . 33

28 Uniaxial tensile testing samples . . . . . . . . . . . . . . 34

29 Uniaxial tensile testing experimental setup . . . . . . . . . . 34

30 Uniaxial tensile testing DIC region of interest . . . . . . . . 35

$31 \quad$ Biaxial tensile cruciform . . . . . . . . . . . . . . 37

$32 \quad$ Biaxial tensile testing experimental setup . . . . . . . . . . . . 37

33 Biaxial tensile testing DIC region of interest . . . . . . . . 38

34 Inflation testing local region . . . . . . . . . . . . 39

35 Inflation testing DIC region of interest . . . . . . . . . . . 39

36 Four-point bend test diagram . . . . . . . . . . . . . . . 40

$37 \quad$ Four-point bend test 3 inch deflection . . . . . . . . . . . . . . 41

38 Four-point bend test DIC camera view of panel top . . . . . . . 41

39 Four-point bend test panel top DIC region of interest . . . . . . 42

$40 \quad$ Four-point bend test DIC camera view of panel bottom . . . . . 42

$41 \quad$ Four-point bend test panel bottom DIC region of interest . . . . 43

42 Uniaxial tension warp and weft load vs position . . . . . . . . 44

43 Uniaxial tension warp sample axial strain field . . . . . . . . . . 45 
44 Uniaxial tension warp sample transverse strain field . . . . . . . 45

45 Uniaxial tension weft sample axial strain field . . . . . . . . . . 46

$46 \quad$ Uniaxial tension weft sample transverse strain field . . . . . . . 46

47 Uniaxial tension warp and weft stress vs strain . . . . . . . . 47

48 Uniaxial tension warp Poisson's ratio . . . . . . . . . . . . . 48

49 Uniaxial tension weft Poisson's ratio . . . . . . . . . . . . . . 48

$50 \quad$ Uniaxial tension $45^{\circ}$ offset sample axial strain field . . . . . . . 49

$51 \quad$ Uniaxial tension $45^{\circ}$ offset sample transverse strain field . . . . 50

52 Uniaxial tension $45^{\circ}$ offset shear stress vs shear strain . . . . 50

53 Biaxial and uniaxial tension load vs position . . . . . . . 51

$54 \quad$ Biaxial tension state of 5 psi inflation pressure axial strain field 52

55 Biaxial tension state of 10 psi inflation pressure axial strain field 52

56 Biaxial tension state of 15 psi inflation pressure axial strain field 53

57 Biaxial tension state of 5 psi inflation pressure transverse strain field ..................... . . 53

58 Biaxial tension state of 10 psi inflation pressure transverse strain field . . . . . . . . . . . . . . . . . . 54

59 Biaxial tension state of 15 psi inflation pressure transverse strain field .................... . . 5 54

60 Biaxial and uniaxial tension stress vs strain . . . . . . . . 55

615 psi inflation pressure axial strain . . . . . . . . . . . 56

$62 \quad 10$ psi inflation pressure axial strain . . . . . . . . . . . 56

$63 \quad 15$ psi inflation pressure axial strain . . . . . . . . . . 57

64 Axial strain profile of local section . . . . . . . . . . 58

655 psi inflation pressure transverse strain . . . . . . . . . . 59 
$66 \quad 10$ psi inflation pressure transverse strain . . . . . . . . . . 59

$67 \quad 15$ psi inflation pressure transverse strain . . . . . . . . . . . 60

68 Transverse strain profile of local section . . . . . . . . . . 60

$69 \quad$ Axial and transverse inflation pressures vs strains . . . . . . . 61

$70 \quad$ Four-point bending panel top surface testing load vs deflection . 62

71 Four-point bending panel top surface deflection at 5 psi inflation

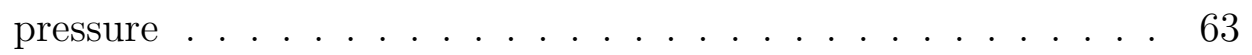

72 Four-point bending panel top surface deflection at 10 psi inflation pressure . . . . . . . . . . . . . . 63

$73 \quad$ Four-point bending panel top surface deflection at 15 psi inflation pressure . . . . . . . . . . . . . . . 64

74 Four-point bending panel top surface axial strain at 5 psi inflation pressure . . . . . . . . . . . . . . 65

75 Four-point bending panel top surface axial strain at 10 psi inflation pressure ................. . . 65

76 Four-point bending panel top surface axial strain at 15 psi inflation pressure . . . . . . . . . . . . 66

77 Four-point bending panel top surface transverse strain at 5 psi inflation pressure ............... 66

78 Four-point bending panel top surface transverse strain at 10 psi inflation pressure . . . . . . . . . . . . 67 67

79 Four-point bending panel top surface transverse strain at 15 psi inflation pressure ............... 67

$80 \quad$ Four-point bending panel bottom surface testing load vs deflection 68

81 Four-point bending panel bottom surface deflection at 5 psi inflation pressure . . . . . . . . . . . . . . 69

82 Four-point bending panel bottom surface deflection at 10 psi inflation pressure ............... 69 
83 Four-point bending panel bottom surface deflection at 15 psi inflation pressure ............... . . . 70

84 Four-point bending panel bottom surface axial strain at 5 psi inflation pressure . . . . . . . . . . . . . 70

85 Four-point bending panel bottom surface axial strain at 10 psi inflation pressure ................ . . 71

86 Four-point bending panel bottom surface axial strain at 15 psi inflation pressure . . . . . . . . . . . . . 71

87 Four-point bending panel bottom surface transverse strain at 5 psi inflation pressure . . . . . . . . . . . . . . 72

88 Four-point bending panel bottom surface transverse strain at 10 psi inflation pressure . . . . . . . . . . . . . 72

89 Four-point bending panel bottom surface transverse strain at 15 psi inflation pressure . . . . . . . . . . . . . 73

$90 \quad$ Summarized material properties . . . . . . . . . . . 75 


\section{LIST OF TABLES}

Table

1

2

3

4

5

6

7

8

Page

Panel build . . . . . . . . . . . . . . . . . . 25

Coordinate system of uniaxial tensile tests . . . . . . . . . 35

Panel inflation stress ratio . . . . . . . . . . . . . 36

Experimentally determined elastic moduli from uniaxial loading 47

Experimentally determined Poisson's ratios from uniaxial loading 49

Experimentally determined elastic moduli from biaxial loading . 55

Experimentally determined elastic moduli from inflation . . . . 61

Four-point bend stiffness and load at maximum deflection of bend tests analyzing the panel top surface . . . . . . . . . . 62

Four-point bend stiffness and load at maximum deflection of bend tests analyzing the panel bottom surface . . . . . . . . 68

Experimentally determined effective modulus from four-point

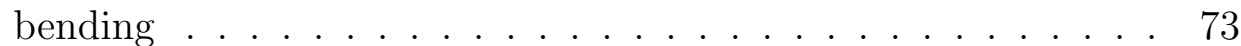




\section{CHAPTER 1}

\section{Introduction}

. In the 1950's a brand new concept called "drop-stitch" technology was explored to improve the performance of inflatable airplanes and small water crafts [9]. Presently drop-stitch technology can be found in modern day space, military, commercial and marine applications. Recent technological advances in fiber and skin materials, coatings, and technical textile processing methods have enabled the next-generation of inflatable structures to achieve significant load-carrying capacities while preserving their minimal weight, rapid and self-erecting deployments and efficient stowed-volume characteristics [10].

\subsection{Drop-stitch inflatable structures}

Drop-stitch technology has an advantage to traditional air-inflated structures because it can achieve flat and curved panel geometries with moderate to large aspect ratios and variable thickness. Drop-stitch fabric construction consists of external skins laminated to a pair of intermediate woven fabric layers separated by a length of perpendicularly aligned fibers which can vary in length depending on the panel geometry. Once inflated, the now tensioned drop-stitch fiber lengths

dictate the thickness of the panel. Complete flatness of the top and bottom faces of the inflated panel can be achieved with a sufficient distribution of drop-stitching. The external skins are typically coated fabrics whose function is to prevent air leakage. This eliminates the need for a separate bladder. [1] 


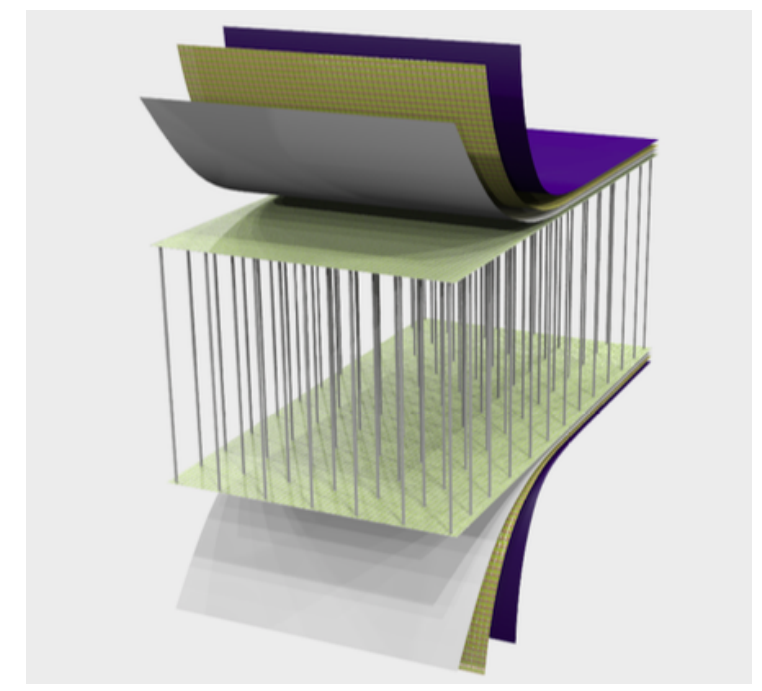

Figure 1: Drop-stitch skin layers configuration [1]

Woven fabrics are commonly considered to be orthotropic materials. This is because for woven structures two main weave directions are defined. The warp direction runs axially along a drop-stitch inflatable panel, while the weft direction runs perpendicular to the warp direction and transversely across a drop-stitch inflatable panel [2].

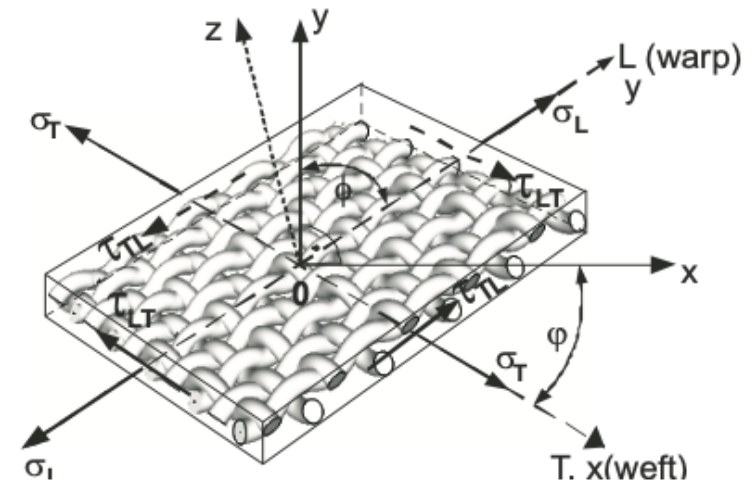

Figure 2: Warp (axial) and weft (transverse) directions [2] 


\subsubsection{Drop-stitch inflatable structure applications}

As stated previously, drop-stitch technology can be found in a variety of fields such as aero-space, military, commercial and marine to name a few.

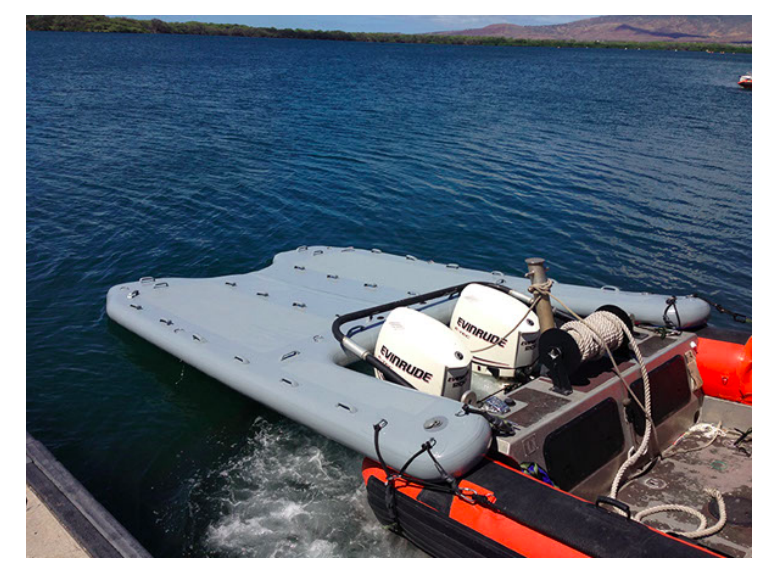

Figure 3: Navatek Inflatable Boat Ramp [3]

Pictured above in Figure 3 is an inflatable boat ramp from Navatek LLC, a company that specializes in drop-stitch inflatable structures. This example is used in a marine application to assist in retrieval when out at sea. When inflated, the ramp can be dropped down off the edge of a vehicle to act as a bridge between the water and vehicle, and when deflated, can be easily pulled back and stored in the vehicle.

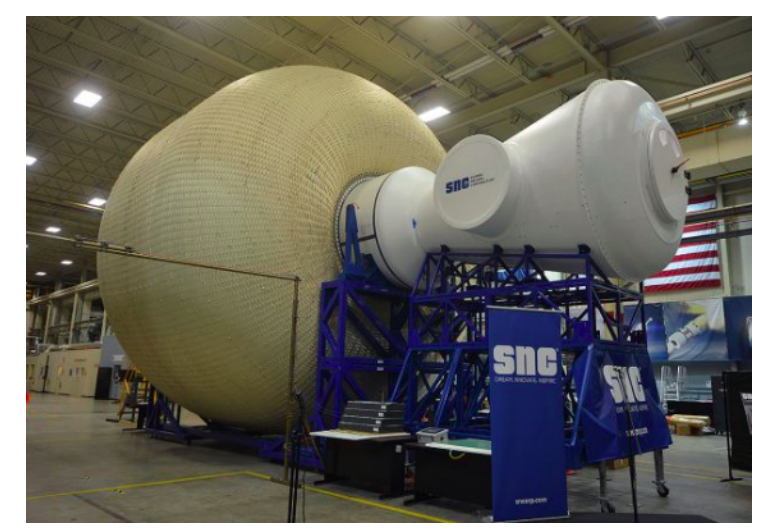

Figure 4: Sierra Nevada Corporation Inflatable Space Habitat [4]

Pictured above in Figure 4 is an inflatable space habitat prototype that the 
Sierra Nevada Corporation fabricated as a prototype for NASA in 2019. The large scale drop-stitch inflatable habitat is compact enough to fit inside an 18-foot rocket fairing but then able to expand to 27 feet in diameter and 27 feet long when deployed and inflated by astronauts in space to use.

\subsection{Motivation}

The challenge in accurately predicting the behaviour of inflatable drop-stitch fabric structures has been due to the complex loading arrangement due to the panels being internally pressurized with air. The panel skin properties in the axial and transverse directions also prove challenging to determine. In a study of air inflated drop-stitch panels being subjected to bending loads it was determined in addition to overall panel stiffness, the panel skin properties in the axial and transverse directions also have an apparent dependence on inflation pressure [11]

Using classical beam theory is the most simple approach to predict the behaviour of a drop-stitch inflatable structure under a four-point loading configuration. Classical beam theory assumes that the cross-sections of the beam remain planar and normal to the mid plane. Classical beam theory also assumes that there is no shear component to the deformation [12].

Air-inflated structures stiffen with increasing pressure and are sensitive to changes in volume produced by transverse shearing deformations of their cross sections. Because classical beam theory requires that plane sections remain planar and normal to the mid plane it neglects transverse shearing deformations. Because of this shear deformable beam theory provides an alternative representation of panel behaviour [11]. For long, slender beams, the shear deformation is very small and can be neglected.

Pressure dependent skin properties are typically determined through correlation with inflated panel bending data. In previous research these skin properties 
have been experimentally determined through stress vs strain curves from uniaxial or biaxial testing machines. This research aims to utilize three dimensional digital image correlation to characterize the material properties under biaxial load conditions designed to simulate stresses induced during bending of inflated drop-stitch panels.

\subsection{Objective of research}

The primary objective of this research is to characterize the constituent material properties and mechanical response of drop-stitch inflatable panels subjected to bending loads. This research aims to characterize the nonlinear elastic response of drop-stitch panels by using a new material system in comparison to previous work. These characterizations included three dimensional digital image correlation, uniaxial and biaxial loading, and panel inflation and bending experiments.

This research is a continuation of previous work done by Smith and Alich based on recommendations for future research. In contrast to previous studies of neoprene drop-stitch inflatable panels, this research utilized a different material system; a commercial PVC panel. This panel was utilized to conduct both inflation and four-point bend tests. In addition to these tests, skin samples were taken from this panel to conduct uniaxial and biaxial skin material tensile testing. All testing utilized three dimensional digital image correlation to provide displacement and strain field data for analysis.

\subsection{Thesis overview}

The purpose of this thesis is to analyze the response of the assumed orthotropic panel skin material and its constituent components utilizing three-dimensional digital image correlation characterizations of uniaxial and biaxial tensile testing, inflation testing and four-point bend testing. 
Chapter 2 will review published literature and previous work done on modeling air-inflated structures and their constituent materials.

Chapter 3 will explain the theory behind this research. This chapter will discuss digital image correlation, mechanics of laminated composites, skin stresses due to inflation and four-point bending. In addition, chapter three will also discuss the preparation, setup and execution of the experiments performed in this research.

Chapter 4 will present the results from the experiments performed in chapter 3 utilizing the theoretical methods and equations also presented earlier in chapter 3. These results will consist of material property calculations in addition to three dimension displacement and strain fields of tensile testing, inflation testing, and four point bend testing of the new material system.

Chapter 5 provides a summary and discussion of the findings from this research, as well as a discussion of the potential topics for future research and experimentation. 


\section{CHAPTER 2}

\section{Literature review}

\section{$2.1 \quad$ Inflatable structures}

A general understanding of the inflation and loading mechanics of inflatable structures is described in technical reports titled "Air-Inflated Fabric Structures" (Cavallaro and Sadegh, 2006) [1] and "Technology and Mechanics Overview of Air-Inflated Fabric Structures" (Cavallaro, 2006) [13]. Both emphasize that the stiffness of the structure is largely dependent on the inflation pressure. As the inflation pressure increases, the skin and fabric layers become stressed and in turn, stiffen the structure. Once external loads are applied to the inflated structure, these loads superpose stress with the inflation stresses already present.

This superposition causes a redistribution of stress along the inflated structure. The redistribution of stress is a natural mechanical response of the structure to balance the load being applied and maintain it's state of static equilibrium. Depending on inflatable structure geometry and loading configuration, the redistribution of stresses can either increase or decrease the tension in the structure skin. If the stresses from the applied external load(s) sufficiently reduce inflation induced stresses, meaning the tension in the skin approaches zero, the onset of wrinkling is expected to occur within the structure. Wrinkling decreases the structure's load carrying capability and leads to a loss in overall stiffnes and eventually collapse of the structure.

In order to better model different inflatable structure geometries and various loading arrangements, it's important to understand the material properties of the structure's skin. A thesis titled "Exploration of the Mechanical Properties of both

Ridged and Inflated drop-stitch Fabric Material" (Felicissimo 2015) [14] from the City University of New York researched these material properties and their non- 
linearities. It was concluded that drop-stitch fabric material is not a homogeneous material with uniform properties throughout. It's also not a standard composite material with varying lamina orientations that produce symmetric property distributions. The inflated structure skin material properties are dependent on both weave direction and also inflation pressure. To properly evaluate the material properties of an inflated structure's skin, each constituent material needs to be tested as a whole and not as individual parts to replicate behaviour in practical applications as best as possible.

\subsection{Inflatable panel beam models}

A desired goal of some inflatable structures research was to have an finite element model successfully model the behavior of an inflated structure. "Bending Behaviour of Plain-Woven Air Beams: Fluid Structure Interaction Approach" (Cavallaro, Sadegh, and Quigley, 2006) [5] attempted to model a plain-woven air beam under four-point bending.

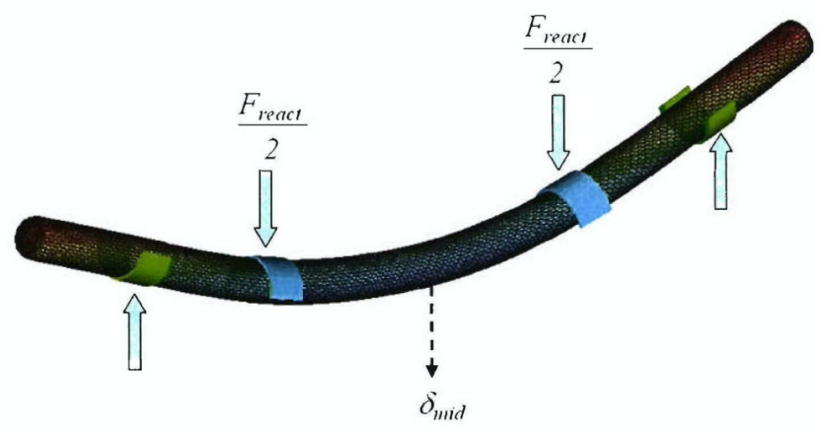

Figure 5: Finite element model of plain-woven air beam four-point bend test [5]

To provide material property inputs to the finite element analysis model, uniaxial and biaxial tensile tests were conducted to experimentally determine the elastic modulus in the longitudinal (axial) direction of the air beam and also the

shear modulus of the skin material. The findings of this study from comparisons of experimental and simulated four-point bend tests were that the structure's volume 
change will be larger at higher inflation pressures because the beam is stiffer at higher pressures and will require a larger load to deflect the beam. This higher external load creates larger shearing forces in the beam. Furthermore, this research found that air compressibility causes a nonlinear stiffening effect in the bending behavior of inflatable structures. As the air volume decreases due to deformations from external loads causing transverse shearing, wrinkling, and section collapse, the internal air pressure will increase. If air compressibility is appreciable, a gas law was suggested to be incorporated in the bending analysis.

Although classical beam theory is one of the most simple methods of modeling the behaviour of drop-stitch inflatable panels, the results of "Bending Tests of Inflatable Drop-stitch Panels" (Falls and Waters, 2011) [15] suggest that shear deformation in these inflated structures is present during bending. The accuracy of the model was tested for panels between $250 \mathrm{~mm}$ and $1000 \mathrm{~mm}$ (9.84 in and $39.37 \mathrm{in}$ ) length, $100 \mathrm{~mm}$ and $200 \mathrm{~mm}$ (3.93 in and $7.87 \mathrm{in})$ thickness, and pressures between $21 \mathrm{kPa}$ and $103 \mathrm{kPa}(3.04$ psi and $14.94 \mathrm{psi})$.

"Mechanics of Air-Inflated Drop-Stitch Fabric Panels Subject to Bending Loads" (Cavallaro, Hart, Sadegh, 2013) [11] also investigated drop-stitch inflatable panels under bending loads. The panels were loaded in a four-point bending configuration and their pressure dependent behaviour was compared to an analytical model that was developed using shear deformable beam theory. As input to the analytical model, uniaxial tensile testing results of the panel skin material in conjunction with Hooke's law were used to establish the biaxial tensile behaviour of the skins.

A thesis titled "Mechanical Response of Polymer-Fabric Skin Materials used in inflatable Drop-Stitch Structures" (Smith, 2019) [16] from the University of Rhode Island aimed to determine if Euler-Bernoulli beam theory was a satisfac- 
tory method for predicting inflatable drop-stitch structure behaviour through the evaluation of panel material properties and panel performance under bending at varying inflation pressures.

Another thesis titled "Modeling, Simulation and Investigation of Inflatable Drop-Stitch Panels with Finite Element Analysis" (Alich, 2019) [6] also from the University of Rhode Island aimed to develop a finite element model of a four-point bending test on an inflatable drop-stitch panel to predict its mechanical response.

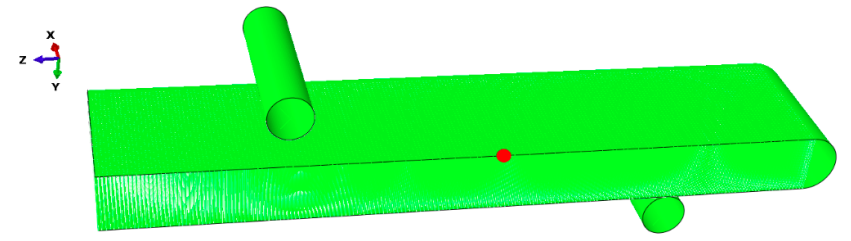

Figure 6: Finite element model of drop-stitch inflatable panel four-point bend test $[6]$

A secondary aim was to validate assumptions and analytical theories that were derived in previous research [16]. Classical lamination theory was used to estimate the orthotropic material properties of the drop-stitch panel skins to use as inputs to a finite element model. The model assumed that skin properties were independent of inflation pressure. Although the finite element model of the panel did not precisely correlate with the experimentally measured mechanical response of the panel, the model did predict increased panel stiffness with increased inflation pressure. The finite element model stress results correlated well to theoretical stresses computed through superposition of inflation pressure based on thin-walled pressure vessel theory and bending stresses from beam bending theory. 


\section{CHAPTER 3}

\section{Methodology}

\subsection{Theory}

\subsubsection{Digital image correlation}

Digital Image Correlation (DIC) is a non-contact optical method of measuring the displacement/deformation of a sample subjected to external loading. The system consists mainly of a digital camera/cameras and specialised computer software. To give a general overview of the process; the cameras capture a consecutive image series of a patterned surface of a test object as it displaces/deforms under an external load. The image series is then processed by a DIC software in which a mathematical correlation analysis of the surface pattern is applied. Finally, displacement/deformation maps for the entire specimen are formed.

In order for the software to efficiently correlate points during the image series of the deformation, the test specimen must be prepared with a random patterning of ideally contrasting regions to it's surface. An initial reference photo of the the patterned surface of the test object prior to external loading and deformation is captured and the software applies a gray scale numerical value to each pixel in the field of view of this reference photo in order to correlate points between the following deformed photos.

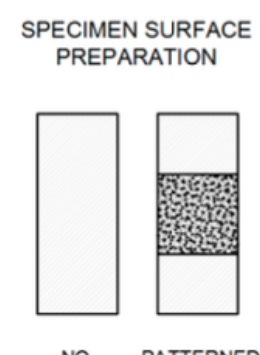

NO
PATTERNED

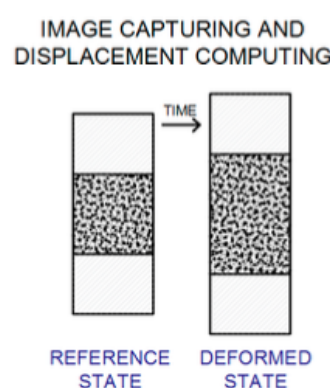

STATE STATE

Figure 7: Digital image correlation surface pattern [7] 
The DIC software groups these gray scale valued pixels into facets to form a virtual grid on the test object surface as shown below in figure 8 .
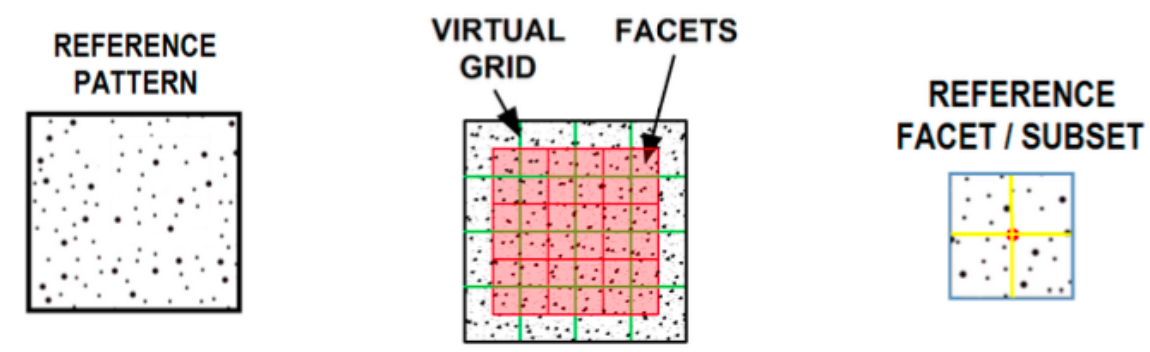

Figure 8: Surface pattern DIC software recognition [7]

The correlation to follow pixels between the reference and deformed photos is then performed by looking for facets with corresponding gray scale value arrangements as shown in 9 .
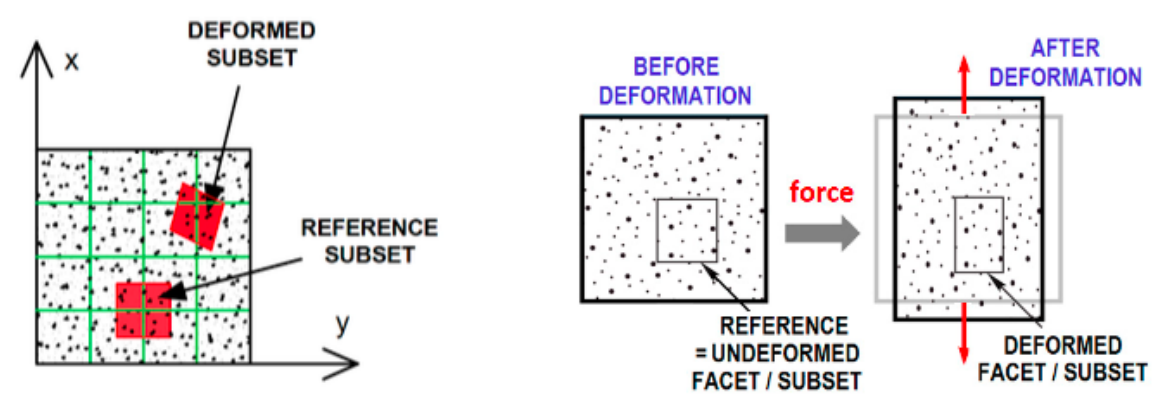

Figure 9: Deformed pattern DIC software recognition [7]

Lastly, by using a correlation algorithm to track the displacement of facets on the surface of the test object as it deforms in the image sequence due to loading, the DIC software generates displacement and strain fields which can then be used for further data analysis.

This research was interested in capturing potential out of plane deformations, so three-dimensional digital image correlation utilizing two cameras was performed. In contrast to two-dimensional digital image correlation, three-dimensional digital image correlation can track non planer movements in relation to the cameras. This 
is due to the pattern being viewed by both cameras and triangulating the position of the facets in a virtual three dimensional coordinate system.
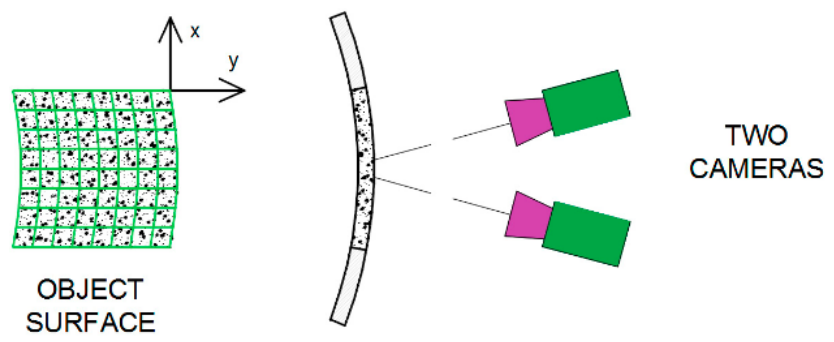

Figure 10: Three dimensional digital image correlation using two cameras [7]

This requires a calibration process of the camera setup to inform the DIC software where the cameras are in relation to one another in this virtual space. The digital image correlation software, camera setups and calibration process specific to the testing performed in the research will be discussed in detail later in the chapter in section 3.2 .

\subsubsection{Mechanics of laminated composites}

The testing performed in this research was designed based on the assumption that the skin material of the drop-stitch panel was an orthotropic continuous fiber-reinforced laminate. Assumed characteristics of continuous fiber reinforced laminates are [17]:

1. The material is constructed of one or more layers, each comprised of fibers that are all uniformly parallel and continuous across the material.

2. The material is in a state of plane stress, i-e., the stresses and strains in the through-the-thickness direction are ignored.

3. The thickness is much smaller than the length and width of the laminate.

A material is considered to be isotropic when it has identical property values in all directions. A material is considered to be orthotropic when material properties 
exhibit three mutually-orthogonal symmetry planes. Orthotropic materials are a subset of anisotropic materials, a group of materials whose material properties change when measured from different directions [12].

It's assumed for the drop-stitch inflatable structure tested in this research that the panel skin is orthotropic, having different material properties in the warp (axial) and weft (transverse) directions.

Hooke's law for an isotropic material under plane stress load conditions is given by

$$
\begin{aligned}
\epsilon_{x} & =\frac{1}{E}\left(\sigma_{x}-\nu \sigma_{y}\right) \\
\epsilon_{y} & =\frac{1}{E}\left(\sigma_{y}-\nu \sigma_{y} x\right) \\
\gamma_{x y} & =\frac{1+\nu}{E} \tau x y
\end{aligned}
$$

where $\mathrm{E}$ is Young's modulus, $\nu$ is Poisson's ratio, $\epsilon_{x}, \epsilon_{y}$ and $\gamma_{x y}$ are the axial, transverse and shear strains and $\sigma_{x}, \sigma_{y}$ and $\tau_{x y}$ are the axial, transverse and shear stresses. For uniaxial loading in the x-direction, the stress-strain relation reduce to

$$
\sigma=E \epsilon
$$

where $\sigma$ and $\epsilon$ are taken to be the stress and strain in the load direction. For an orthotropic material under uniaxial loading in the warp or weft directions, the stress strain relations are given by

$$
\begin{aligned}
& \sigma_{w a r p}=E_{w a r p} \epsilon_{w a r p} \\
& \sigma_{w e f t}=E_{w e f t} \epsilon_{w e f t}
\end{aligned}
$$

The warp (axial) direction will be notated with a subscript 1 while the weft (transverse) direction will be notated with a subscript 2, reducing equation 3 to 


$$
\begin{gathered}
\sigma_{1}=E_{1} \epsilon_{1} \\
\sigma_{2}=E_{2} \epsilon_{2}
\end{gathered}
$$

Strains in the axial and transverse directions are related through Poisson's ratio. Poisson's ratio associated with lateral contraction in the weft direction due to loading in the warp direction is given by

$$
\nu_{12}=-\frac{\epsilon_{2}}{\epsilon_{1}}
$$

while Poisson's ratio associated with lateral contraction in the warp direction due to loading in the weft direction is given by

$$
\nu_{21}=-\frac{\epsilon_{1}}{\epsilon_{2}}
$$

The strains in the axial and transverse directions can now be related and written as the strain in the direction of an applied force minus the contraction of Poisson's effect.

$$
\begin{aligned}
& \epsilon_{1}=-\frac{\sigma_{1}}{E_{1}}-\nu_{21} \epsilon_{2} \\
& \epsilon_{2}=-\frac{\sigma_{2}}{E_{2}}-\nu_{12} \epsilon_{1}
\end{aligned}
$$

Substituting equations 4 into equations 7 yields

$$
\begin{aligned}
\epsilon_{1} & =-\frac{\sigma_{1}}{E_{1}}-\nu_{21} \frac{\sigma_{2}}{E_{2}} \\
\epsilon_{2} & =-\frac{\sigma_{2}}{E_{2}}-\nu_{12} \frac{\sigma_{1}}{E_{1}}
\end{aligned}
$$

Equation 8 can be rearranged to solve for axial and transverse moduli, giving the result

$$
\begin{array}{r}
E_{1}=\frac{\sigma_{1}}{\epsilon_{1}+\nu_{21} \frac{\sigma_{2}}{E_{2}}} \\
E_{2}=\frac{-\nu_{21} \sigma_{2}}{\epsilon_{1}-\frac{\sigma_{1}}{E_{1}}}
\end{array}
$$


and

$$
\begin{array}{r}
E_{1}=\frac{-\nu_{12} \sigma_{1}}{\epsilon_{2}-\frac{\sigma_{2}}{E_{2}}} \\
E_{2}=\frac{\sigma_{2}}{\epsilon_{2}+\nu_{12} \frac{\sigma_{1}}{E_{1}}}
\end{array}
$$

Equating equations 9 and 10 gives equation 11.

$$
\begin{aligned}
& E_{1}=\frac{\sigma_{1}}{\epsilon_{1}+\nu_{21} \frac{\sigma_{2}}{E_{2}}}=\frac{-\nu_{12} \sigma_{1}}{\epsilon_{2}-\frac{\sigma_{2}}{E_{2}}} \\
& E_{2}=\frac{-\nu_{21} \sigma_{2}}{\epsilon_{1}-\frac{\sigma_{1}}{E_{1}}}=\frac{\sigma_{2}}{\epsilon_{2}+\nu_{12} \frac{\sigma_{1}}{E_{1}}}
\end{aligned}
$$

Rearranging equation 11 gives expressions for the elastic modulus of an orthotropic laminated composite in the warp (axial) and weft (transerse) directions.

$$
\begin{aligned}
& E_{1}=\frac{\sigma_{1}\left(\nu_{21} \nu_{12}-1\right)}{-\nu_{21} \epsilon_{2}-\epsilon_{1}} \\
& E_{2}=\frac{\sigma_{2}\left(\nu_{12} \nu_{21}-1\right)}{-\nu_{12} \epsilon_{1}-\epsilon_{2}}
\end{aligned}
$$

Equation 12 was used to calculate elastic moduli values of the drop-stitch inflatable panel skin from a state of plane stress induced by a biaxial load frame. Load values were recorded by the load frame and later converted to stresses $\sigma_{1}$ and $\sigma_{2}$, strains $\epsilon_{1}$ and $\epsilon_{2}$ were measured from digital image correlation, and the Poisson's ratios $\nu_{12}$ and $\nu_{21}$ were calculated from uniaxial testing using equations 5 and 6 .

Shear stresses and strains are related by an in-plane shear modulus, $G_{12}$ through the relation

$$
\tau_{12}=G_{12} \gamma_{12}
$$

ASTM 3518 [8] which was adopted from the work of Rosen [18] previously, describes a method of determining the shear modulus of a composite through uniaxial tensile testing of a $45^{\circ}$ offset sample. The sample described is shown in Figure 11. 


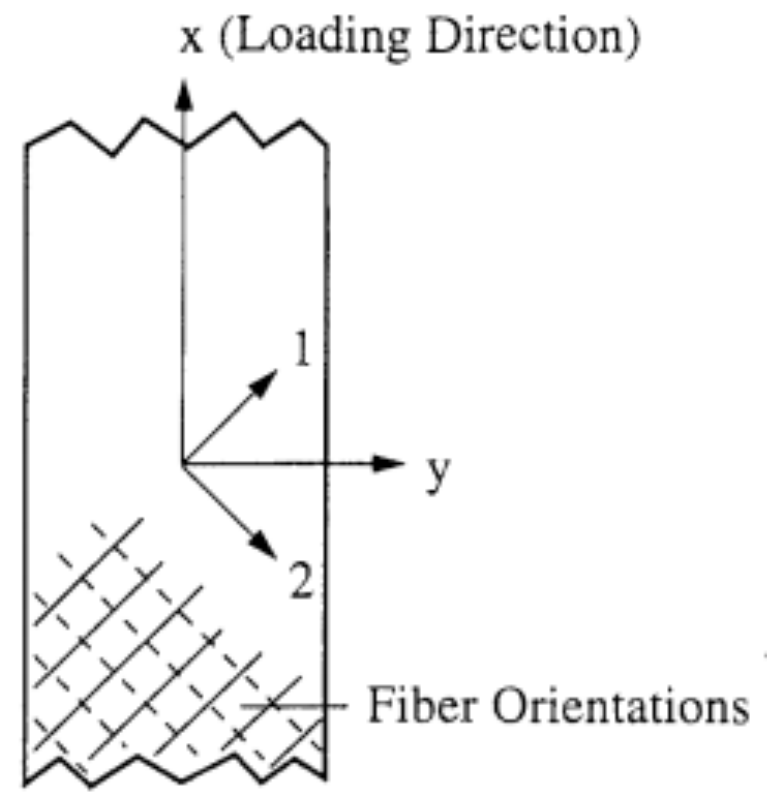

Figure 11: $45^{\circ}$ offset sample fiber orientation [8]

Following the coordinate system shown, uniaxial loading applied in the $\mathrm{x}$ direction induces strains in the axial and transverse directions, $\epsilon_{x}$ and $\epsilon_{y}$, respectively. Under these load conditions, the shear modulus associated shear parallel to the fiber directions can be shown to be given by

$$
G_{12}=\frac{\sigma_{x}}{2\left(\epsilon_{x}-\epsilon_{y}\right)}
$$

Equation 14 was used to calculate the shear modulus of the drop-stitch inflatable panel skin from a $45^{\circ}$ offset sample in a state of uniaxial stress induced by one axis of a biaxial load frame. Load values were recorded by the load frame and later converted to stress, and strains were measured from digital image correlation.

Upon determining material property values using equations 4, 5, 6, and 14 a compliance matrix can be formed as shown in equation 15 .

$$
\left[\begin{array}{c}
\epsilon_{1} \\
\epsilon_{2} \\
\gamma_{12}
\end{array}\right]=\left[\begin{array}{ccc}
S_{11} & S_{12} & 0 \\
S_{21} & S_{22} & 0 \\
0 & 0 & S_{66}
\end{array}\right]\left[\begin{array}{c}
\sigma_{1} \\
\sigma_{2} \\
\tau_{12}
\end{array}\right]
$$

where the constants given by $\mathrm{S}$ are: 


$$
\begin{array}{r}
S_{11}=\frac{1}{E_{1}} \\
S_{22}=\frac{1}{E_{2}} \\
S_{12}=S_{21}=\frac{-\nu_{12}}{E_{1}}=\frac{-\nu_{21}}{E_{2}} \\
S_{66}=\frac{1}{G_{12}}
\end{array}
$$

Inverting the compliance matrix gives stress as a function of strain as shown in equation 17.

$$
\left[\begin{array}{c}
\sigma_{1} \\
\sigma_{2} \\
\tau_{12}
\end{array}\right]=\left[\begin{array}{ccc}
Q_{11} & Q_{12} & 0 \\
Q_{21} & Q_{22} & 0 \\
0 & 0 & Q_{66}
\end{array}\right]\left[\begin{array}{c}
\epsilon_{1} \\
\epsilon_{2} \\
\gamma_{12}
\end{array}\right]
$$

where the constants given by $\mathrm{Q}$ are:

$$
\begin{aligned}
& Q_{11}=\frac{E_{1}}{1-\nu_{12} \nu_{21}} \\
& Q_{22}=\frac{E_{2}}{1-\nu_{12} \nu_{21}} \\
& Q_{12}=Q_{21}=\frac{\nu_{12} E_{2}}{1-\nu_{12} \nu_{21}}= \frac{\nu_{21} E_{1}}{1-\nu_{12} \nu_{21}} \\
& Q_{66}=G_{12}
\end{aligned}
$$

\subsubsection{Inflation stress}

Using the same approach as Alich [6], thin pressure vessel theory can be applied to drop-stitch inflatable panels to calculate the stresses in the panel's skin due to inflation. By considering equilibrium between the inflation pressure and the stresses in the panel skin leads to the relation

$$
P A_{1}=\sigma_{\text {skin }} A_{2}
$$

Equation 20 states the product of internal inflation pressure multiplied by the cross sectional area of the internal cavity of the panel must equal the stress in the panel skin multiplied by the cross sectional area of the panel skin. Figure 12 shows the axial and transverse areas and applicable dimensions. 


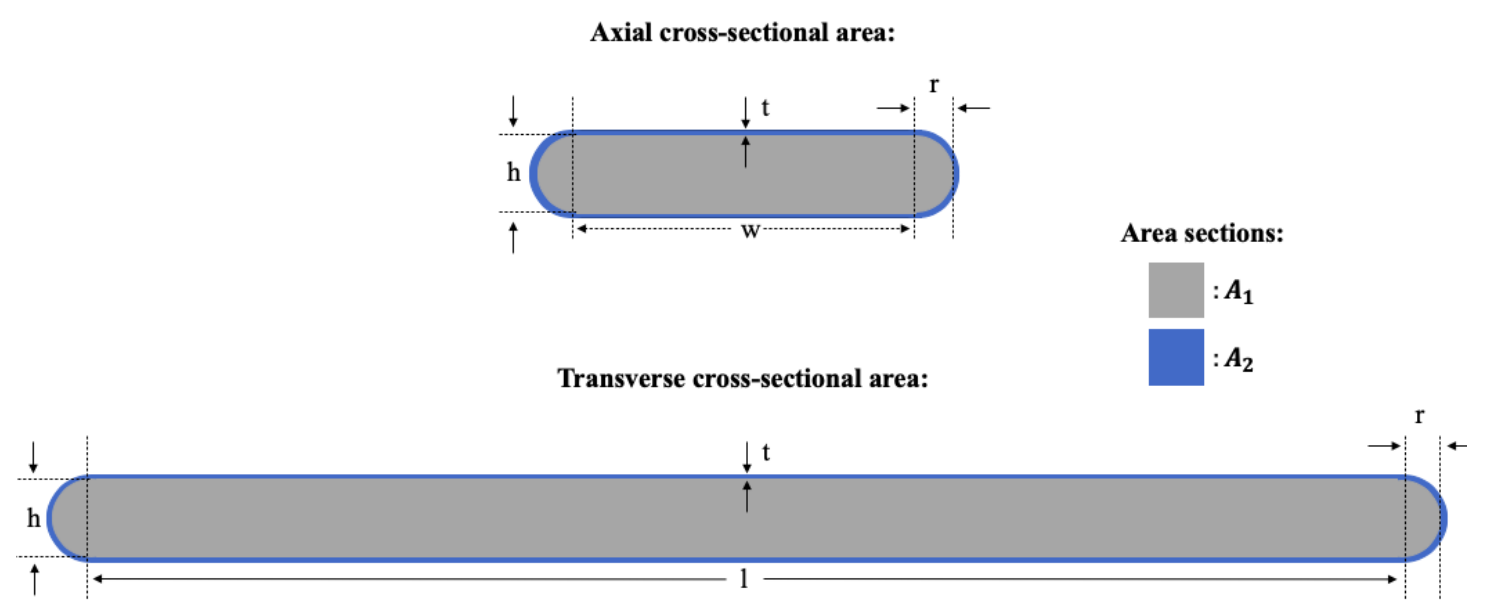

Figure 12: Axial and transverse cross-sectional areas

The cross sectional area of the internal cavity between skin layers of the panel can be calculated by finding the area of the central region, and then adding the area of the two side walls. For the axial cross sectional area, the central region is given by the panel width times the height, and for the transverse cross-sectional area, the central region is given by the panel length times the height. Equations 21 are the axial and transverse cross-sectional areas of the internal cavity, respectively.

$$
\begin{gathered}
A_{1, \text { axial }}=h w+\pi r^{2} \\
A_{1, \text { transverse }}=h l+\pi r^{2}
\end{gathered}
$$

The cross sectional area of the panel skin can again be found by finding the area in the panel's central region, and then adding the side wall skin cross sectional areas. For the axial skin cross sectional area, the central region is made up of two skins of area width times thickness. For the transverse skin cross sectional area, the central region is made up of two skins of area; length times thickness. The sidewall skin cross sectional areas are assumed to be the same in the axial and transverse directions. This side wall skin area can be calculated by finding the area of of a circle with radius $r$ plus the thickness of the panel skin, minus the area of a circle with radius r. Equations 22 are the axial and transverse cross-sectional 
areas of the panel skin, respectively.

$$
\begin{array}{r}
A_{2, \text { axial }}=2 t w+\pi\left((r+t)^{2}-r^{2}\right) \\
A_{2, \text { transverse }}=2 t l+\pi\left((r+t)^{2}-r^{2}\right)
\end{array}
$$

Substituting equations 21 and 22 into equation 20 for axial and transverse directions and rearranging yields equations 23 which are the stresses due to inflation of the drop-stitch panel used in this research.

$$
\begin{aligned}
\sigma_{\text {axial }} & =\frac{P\left(h w+\pi r^{2}\right)}{2 t w+\pi\left((r+t)^{2}-r^{2}\right)} \\
\sigma_{\text {transverse }} & =\frac{P\left(h l+\pi r^{2}\right)}{2 t l+\pi\left((r+t)^{2}-r^{2}\right)}
\end{aligned}
$$

\subsubsection{Four-point bending}

The reason four point bending is the preferred loading configuration for testing inflatable drop-stitch panels is because four-point bending generates a region at the mid span of the panel of pure bending and no shear transverse stress which occurs locally under the loading pins. This is shown in the shear and moment diagrams of a conventional structure in figure 13 [11]. 


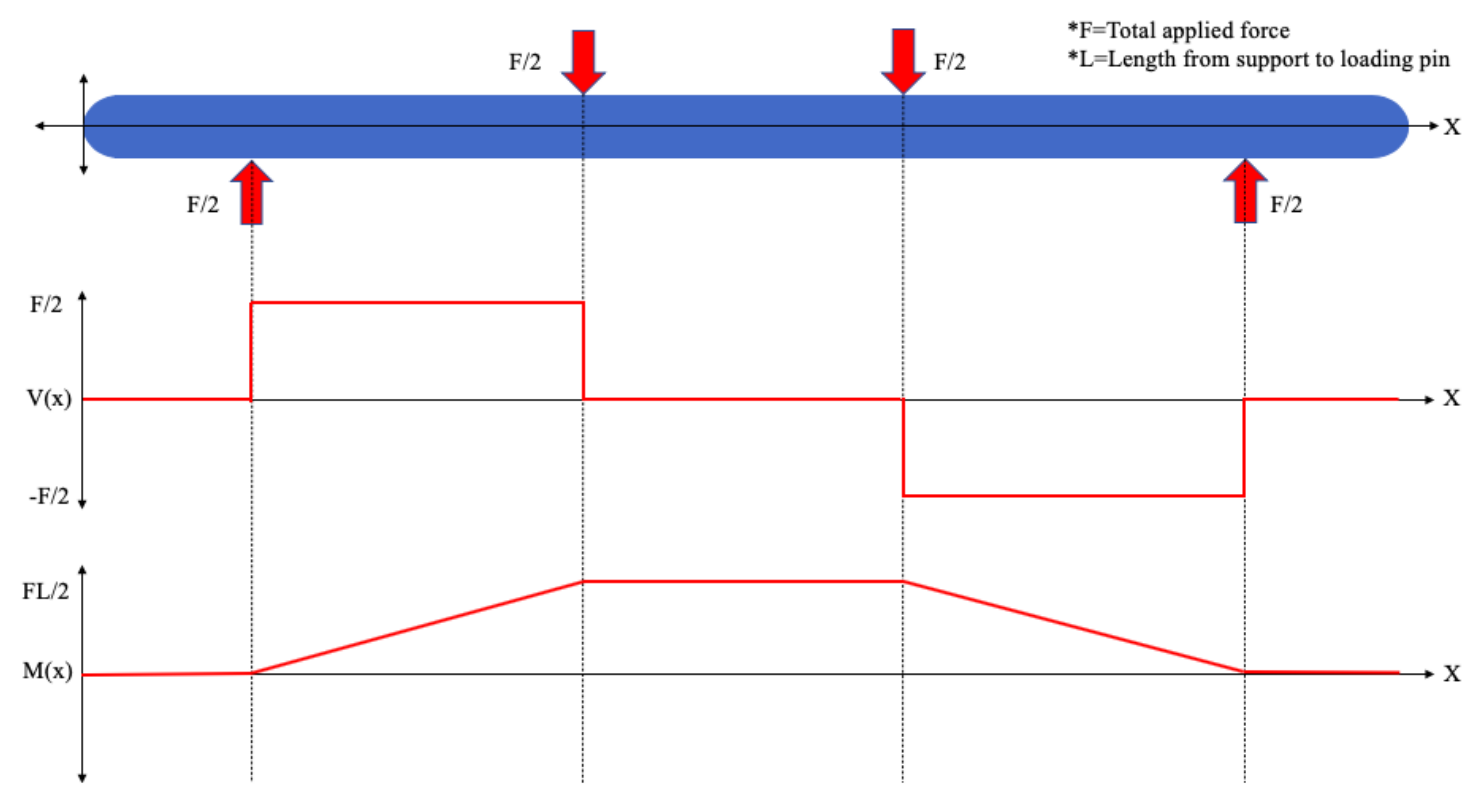

Figure 13: Four-point bending shear and moment diagrams

However due to the internal inflation pressure of drop-stitch inflatable panels, conventional stresses due to four-point bending are not the only stresses present. The panel skin is pre-tensioned due to inflation, and these stresses superpose with bending stresses as the panel deflects [6]. This is shown in the bending stress diagram in figure 14.

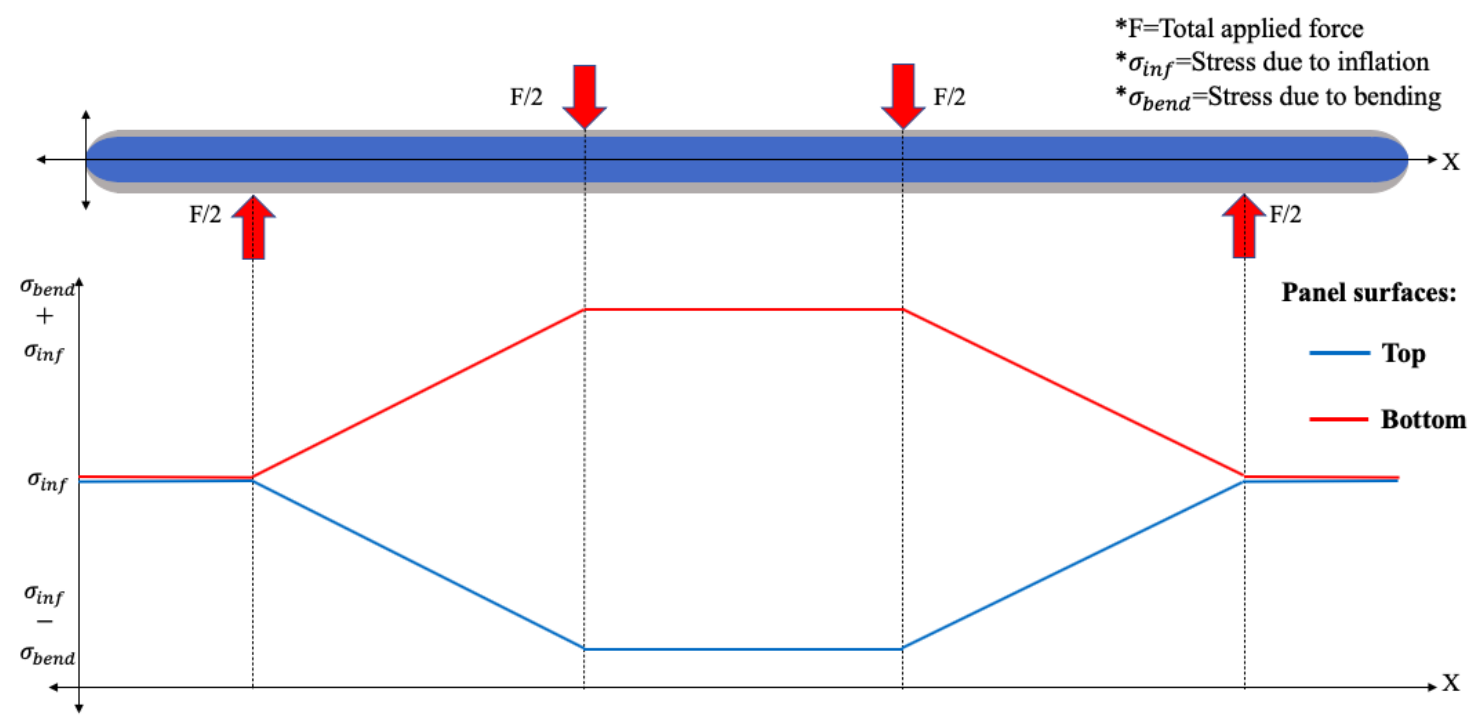

Figure 14: Four-point bending panel stress diagram 
It should be noted that for the panel top surface when bending stresses are superposed with inflation stresses, the bending stresses are compressive so they are subtracted from the inflation stresses, while the opposite happens on the panel bottom surface. The bending stresses are now tensile so when the stresses are superposed the bending stresses are added to the inflation stresses.

According to Roylance [19], if the relation between the applied load and the deflection of a beam bending experiment is known, it's possible to determine elastic modulus from the measurement. A stiffness measured this way is called the flexural modulus.

For a beam under a symmetrical four-point bending load, the deflection anywhere along the span of the beam is given by equation 24

$$
\begin{gathered}
\delta(x)=\frac{\frac{F}{2}(L-a)}{6 L E I}\left[\frac{L}{L-a}(x-a)^{3}-x^{3}+\left(L^{2}-(L-a)^{2}\right) x\right] \ldots \\
\ldots+\frac{\frac{F}{2} a}{6 L E I}\left[\frac{L}{a}(x-(L-a))^{3}-x^{3}+\left(L^{2}-a^{2}\right) x\right]
\end{gathered}
$$

where $\mathrm{x}$ is the position along the span of the beam, $\mathrm{F}$ is the total applied load, $\mathrm{L}$ is the distance between supports, a is the distance from the support to the load, $\mathrm{E}$ is the flexural modulus in the axial direction and $\mathrm{I}$ is the moment of inertia.

Using a similar approach to Smith [16], the moment of inertia of the axial cross-sectional area of the drop-stitch inflatable panel used in this research can be calculated using the parallel axis theorem. Figure 15 shows how the cross section can be approximated as two rectangles and two semi-circles. The panel top and bottom surfaces are represented by rectangles of width $\mathrm{w}$, the sidewalls are represented by semi circles of radius $\mathrm{r}$, and the panel has height $\mathrm{h}$. Uniform thickness is assumed throughout the cross section. 


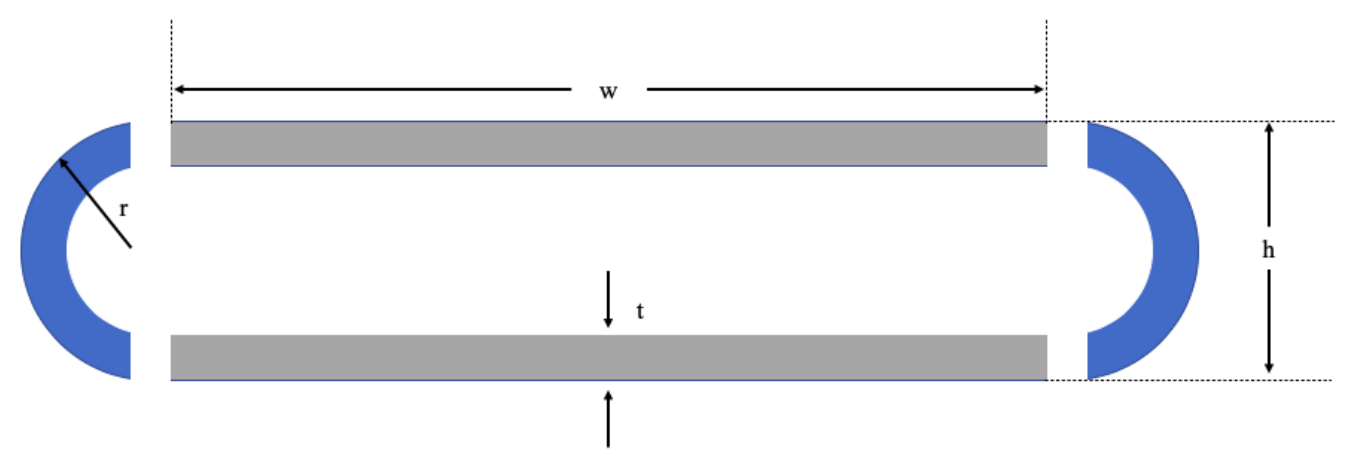

Figure 15: Panel cross section geometry simplification

The approximated moment of inertia of the panel cross section is given by equation 25.

$$
I=I_{\text {top } / \text { bottom }}+I_{\text {sidewall }}
$$

The moment of inertia of the top/bottom rectangle is given by equation 26

$$
I_{\text {top } / \text { bottom }}=\frac{w t^{3}}{12}+w t\left(\frac{h}{2}+\frac{t}{2}\right)^{2}
$$

and the moment of inertia of the semi circles is given by equation 27 .

$$
I_{\text {sidewall }}=\frac{\pi}{8}\left[(r+t)^{4}-r^{4}\right]
$$

Note that equations 26 and 27 only give the moment of inertia of one panel surface and one sidewall. Thus these equations are multiplied by two and then substituted back into equation 25 yielding equation 28

$$
I=\frac{w t^{3}}{6}+2 w t\left(\frac{h}{2}+\frac{t}{2}\right)^{2}+\frac{\pi}{4}\left[(r+t)^{4}-r^{4}\right]
$$

which is the approximated moment of inertia of the panel cross section.

Knowing that the maximum experimental deflection of the drop-stitch inflatable panel was 3 inches, equation 24 can be used with the corresponding total applied load measured by the test frame load cell to determine apparent material property values from the four-point bending tests. 


\subsection{Experimental preparation and setup}

\subsubsection{Drop-stitch inflatable panel build}

As stated previously, this research aims to characterize the nonlinear elastic response of drop-stitch panels by using a new material system in comparison to previous studies. These experiments evaluated a commercial PVC panel as show in Figure 16.

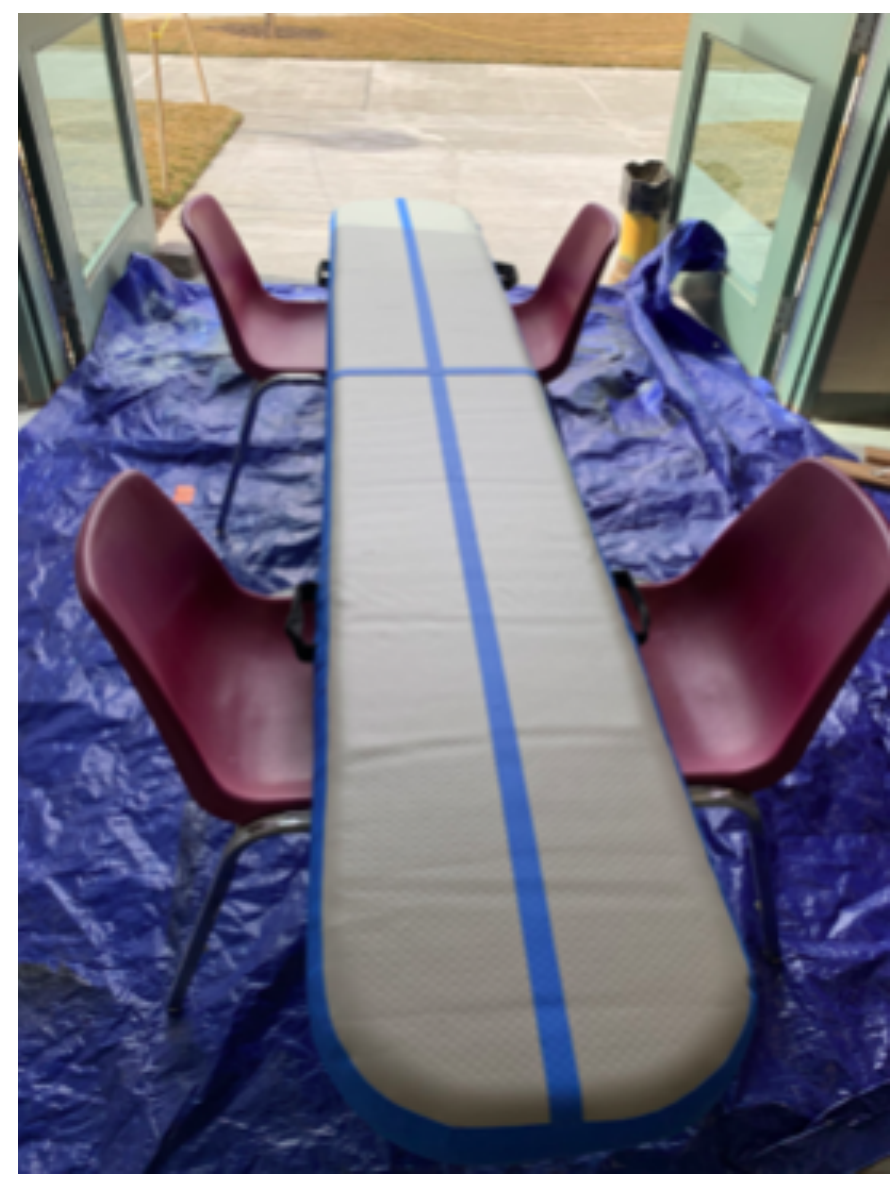

Figure 16: Drop-stitch inflatable panel

The panel is made up of four sections/layers as shown in 17. Layer one is an outer blue tarpaulin-PVC Layer which makes up the sidewall and seam taping on the top surface. Layer two is a grey core tarpaulin-PVC layer which makes up the panel top and bottom surfaces. Layer three is a woven fabric laminated to the panel PVC skin, and section four contains drop-stitches inside the panel that connect 
the top and bottom surfaces to give the panel it's flat geometry when inflated. It should be noted that the top and bottom fabrics are woven simultaneously with periodic exchanges of drop stitch yarns.

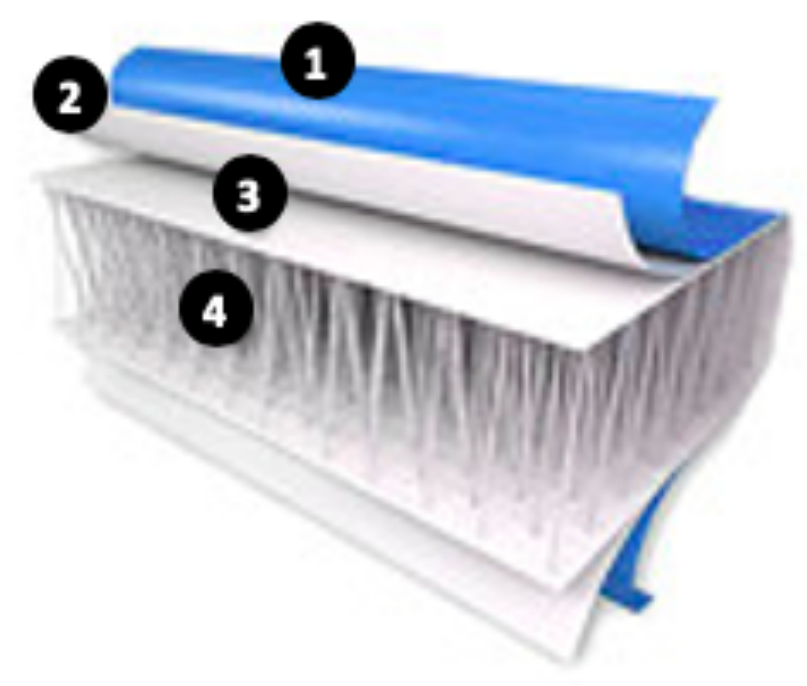

Figure 17: Drop-stitch inflatable panel skin layers

Table 1 summarizes the panel's build characteristics with common textile industry measurements of drop-stitch inflatable structures.

Table 1: Panel build

\begin{tabular}{c|c} 
Build characteristic & Commercial panel \\
\hline Outer coating material & $P V C$ \\
Coating (panel skin) thickness (in) & 0.05 \\
Drop yarn density (yarns/in ${ }^{2}$ ) & 5 \\
Panel inflated length (in) & 120 \\
Panel inflated width (in) & 20 \\
Panel inflated thickness (in) & 4
\end{tabular}

All drop-stitch inflatable structures contain a fabric layer laminated to the outer skin coatings. These layers are typically woven in two orthogonal directions denoted as warp and weft. Figure 18 shows a microscopic view of the commercial drop-stitch panel's fabric layer. As shown, the fabric is a knitted with yarns having 
overall orientations in orthogonal directions. Yarns oriented in the axial direction of the drop-stitch panel are identified as warp fibers and those in the transverse direction of the drop-stitch panel are identified as weft fibers. The panel skin is expected to exhibit orthotropic behavior under loading.

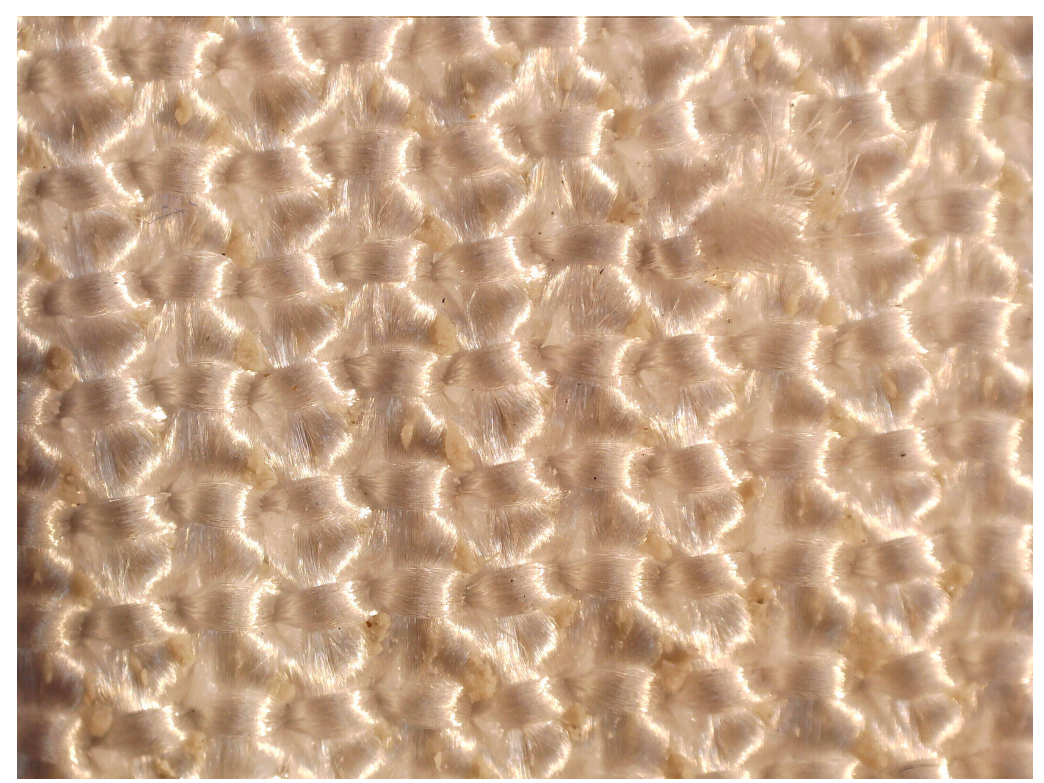

Figure 18: Drop-stitch panel fabric

The panels are inflated using an air compressor. The commercial PVC panel was manufactured with a standard valve used in inflatable structures called a Halkey-Roberts valve. Also commonly referred to as an HR valve, they are a oneway check valve that makes inflation and deflation of inflatable structures as quick as possible. Figure 19 shows the Halkey-Roberts valve located on the side wall of the commercial drop-stitch panel used in this research. 


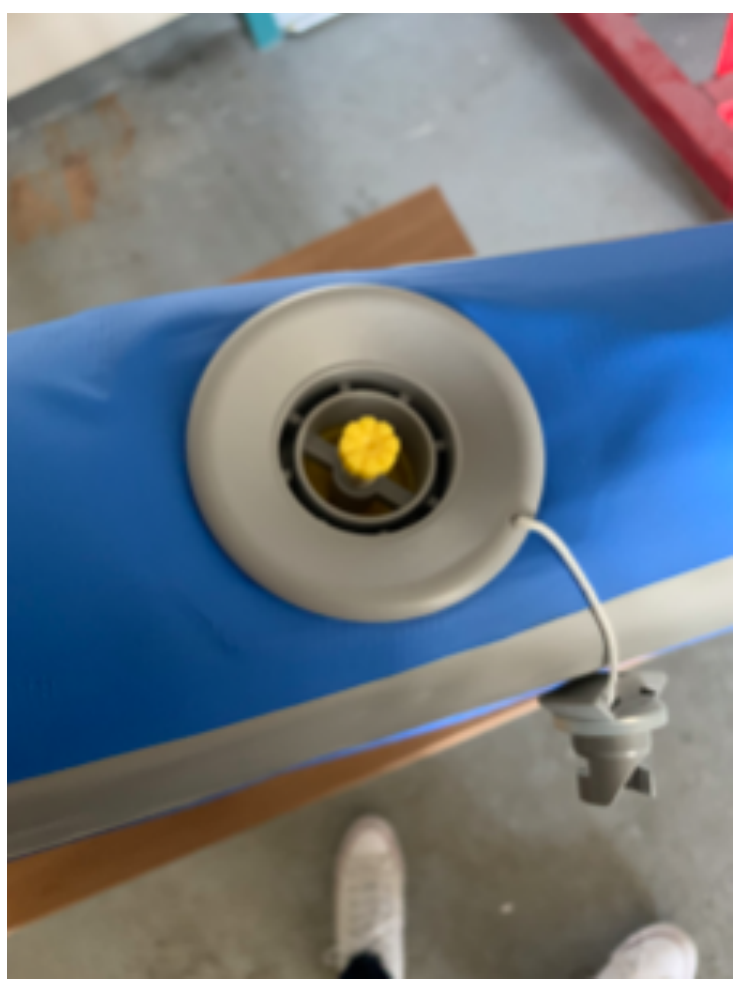

Figure 19: Halkey-Roberts valve

The corresponding Halkey-Roberts valve adapter used to inflate the panel can be seen in Figure 20.

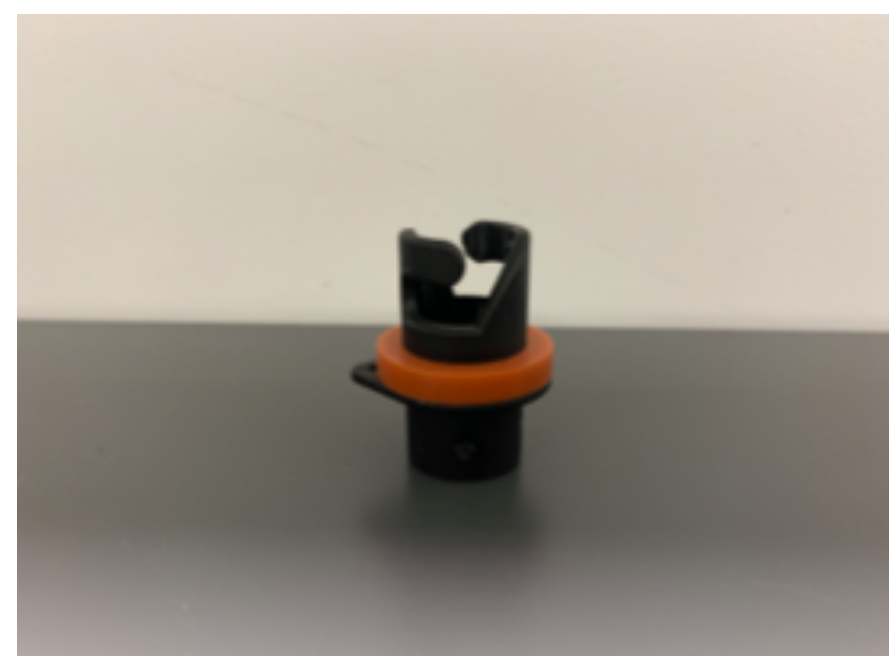

Figure 20: Halkey-Roberts valve adapter 


\subsubsection{Digital image correlation}

In order to utilize three dimensional DIC to analyze experimental loading of the drop-stitch panel and it's constituent materials, the surfaces of these test samples needed to be prepared with proper patterning for accurate facet tracking through a series of images. In order to achieve maximum contrast between the outer PVC skin layer of the panel and the pattern, a white base coat was applied using spray paint as shown below in Figure 21.

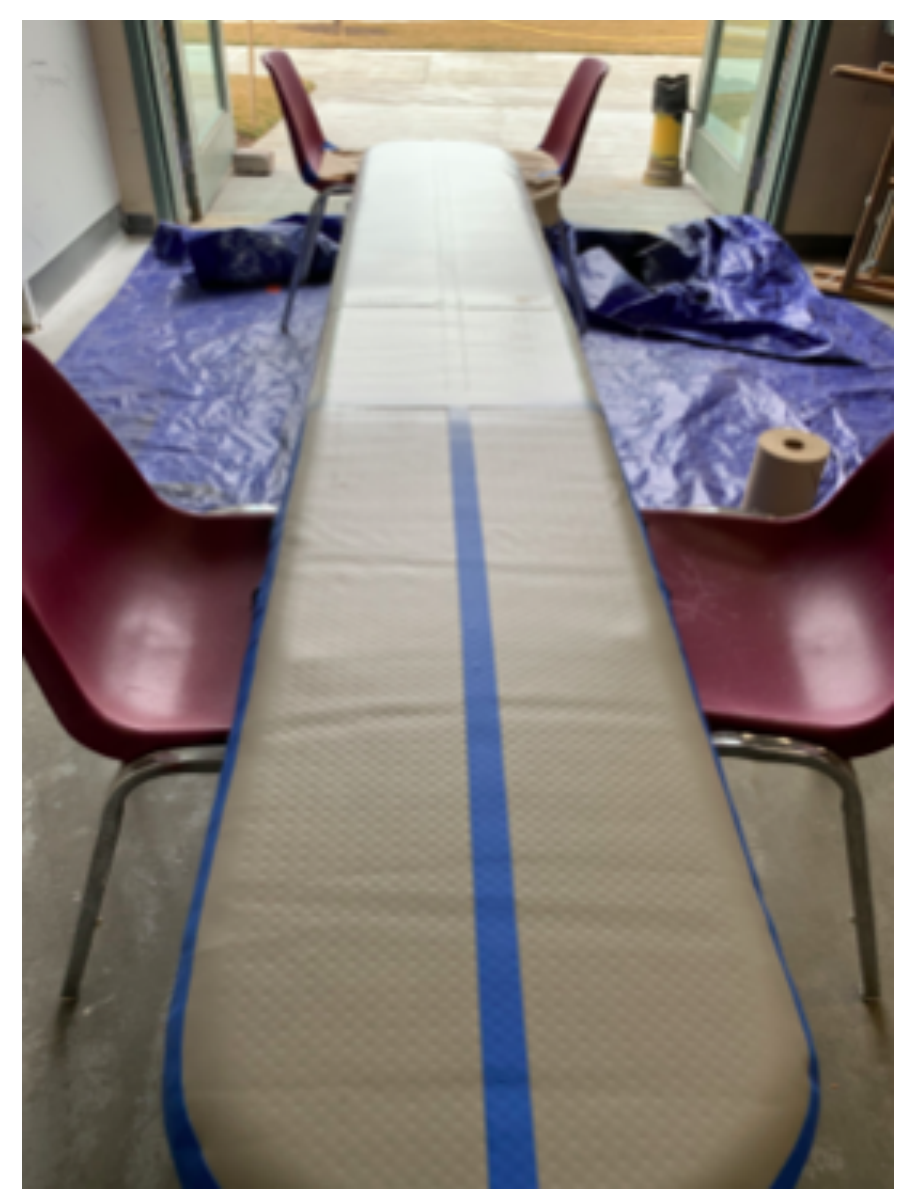

Figure 21: Panel four-point bend test white base coat

Then, using black spray paint, a random speckle pattern was applied on top of this white base coat as shown in Figure 22. 


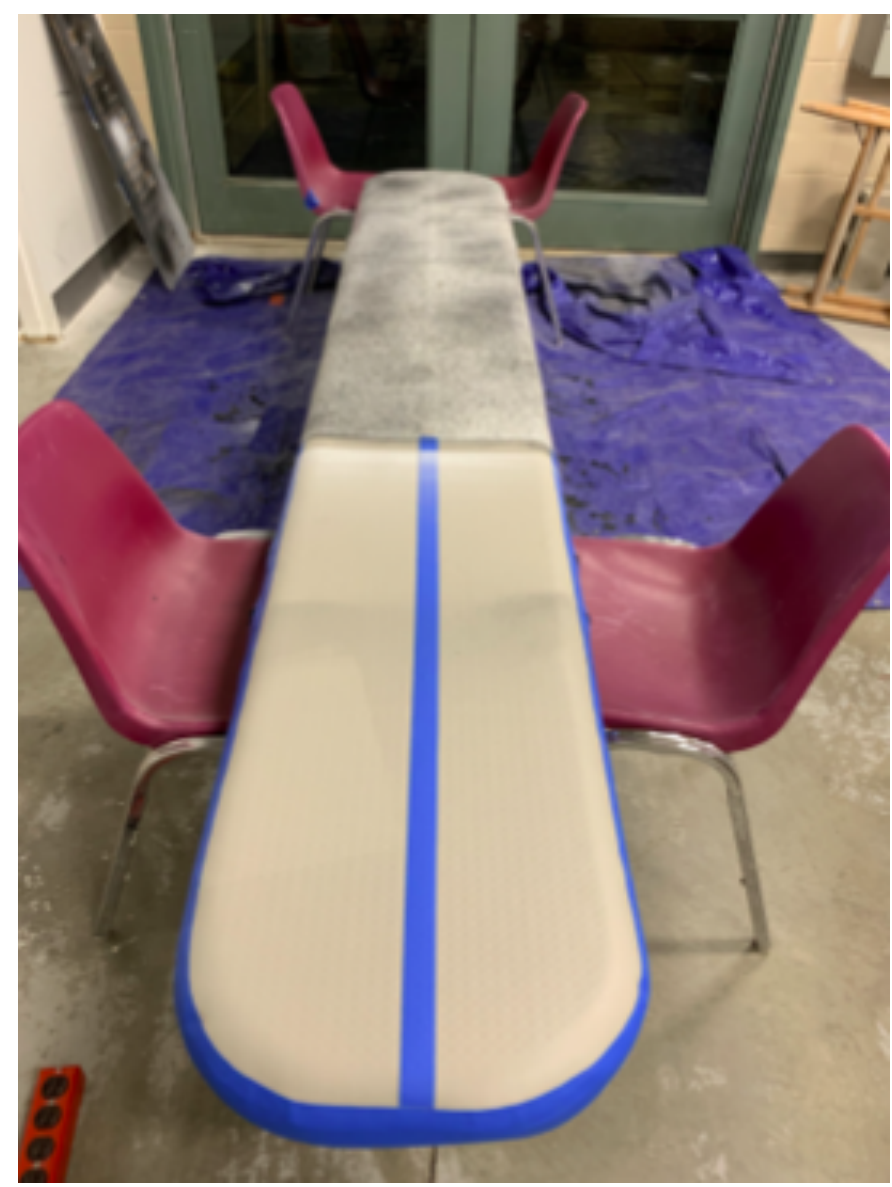

Figure 22: Panel four-point bend test full speckled section of panel

Notice that the base coat and speckle pattern were applied from the end of the panel to slightly past it's midpoint. This was done on the panel top, bottom and one side wall in order to analyze compressive strains on the panel top and strains in tension on the panel bottom while it was loaded in four point bending.

Figure 23 shows a close up view of the speckle pattern applied to panel skin. As you can see this region is about fifty percent white base coat and fifty percent black pattern which is best practice to achieve accurate pattern tracking with digital image correlation. Also, black speckle pattern is very random and nonlinear which is important to allow the software to properly distinguish between facets and track them through the image series. Furthermore, there should ideally be three to five speckles per facet for accurate tracking between images. Because the field of view 
of the panel was so large the size of the speckles had to be proportionally large. The size of these speckles was produced by holding the spray paint nozzle partially down and sputtering the spray paint as opposed to the normal mist produced when the nozzle is held fully down.

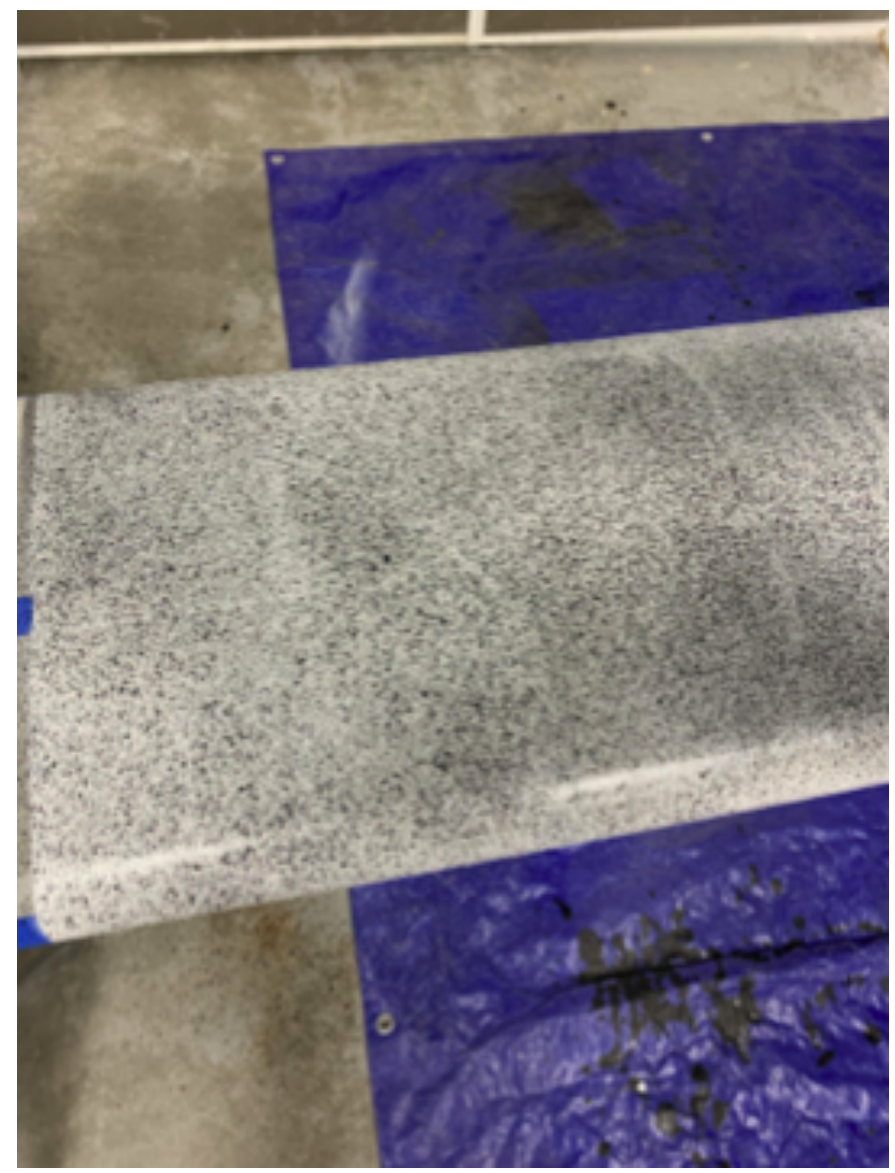

Figure 23: Panel four-point bend test speckle pattern

An identical speckling process to that described for the drop-stitch panel was used to prepare the other test samples of this research for digital image correlation. Figure 24 shows both uniaxial and biaxial tensile testing samples prepared for digital image correlation. Notice that the speckle pattern on these samples are much finer than that of the panel shown previously. This is because the field of view for tensile testing was much smaller and closer compared to that of four point bend testing the drop-stitch panel. In order to still achieve three to five speckles 
in a single facet this fine speckle pattern was applied by misting the black spray paint over the white base coat.

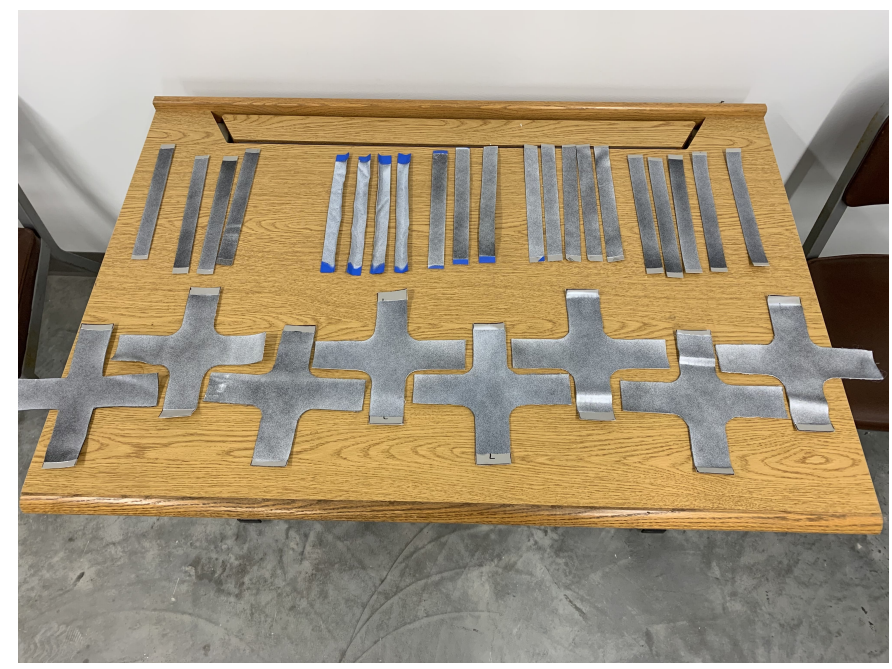

Figure 24: Biaxial and uniaxial tensile test speckle patterns

Similar to tensile testing, the field of view for analyzing a local region of the panel skin as it inflates was very small. A fine speckle pattern was applied as shown in figure 25.

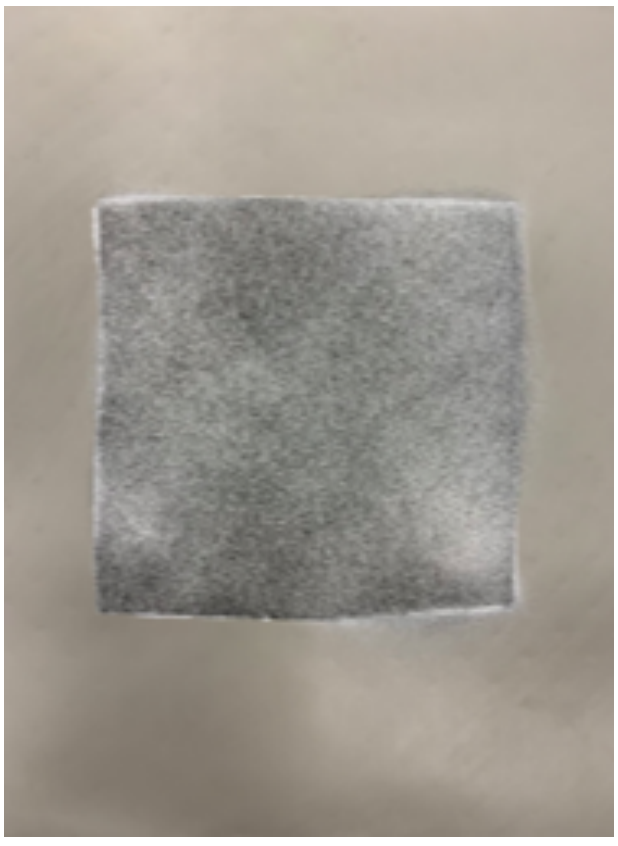

Figure 25: Inflation test speckle pattern 
Once the surfaces were prepared, the cameras were setup in order to achieve the desired field of view for each test. It was key to make sure the the measuring volume was also lit using external light sources to aid in the contrast between the base coat and pattern. The focus and aperture settings of the cameras were then adjusted appropriately.

As mentioned previously, it's very important when performing three dimensional digital image correlation for the software to know where each camera is in relation to each other in order to properly triangulate the position of the facets in the measuring volume. This is achieved by calibrating the camera setup. This was done using the calibration board for smaller fields of view in tensile testing and inflation testing, while using the calibration cross for a larger field of view in four-point panel bend testing as shown in Figures 26 and 27 respectively.

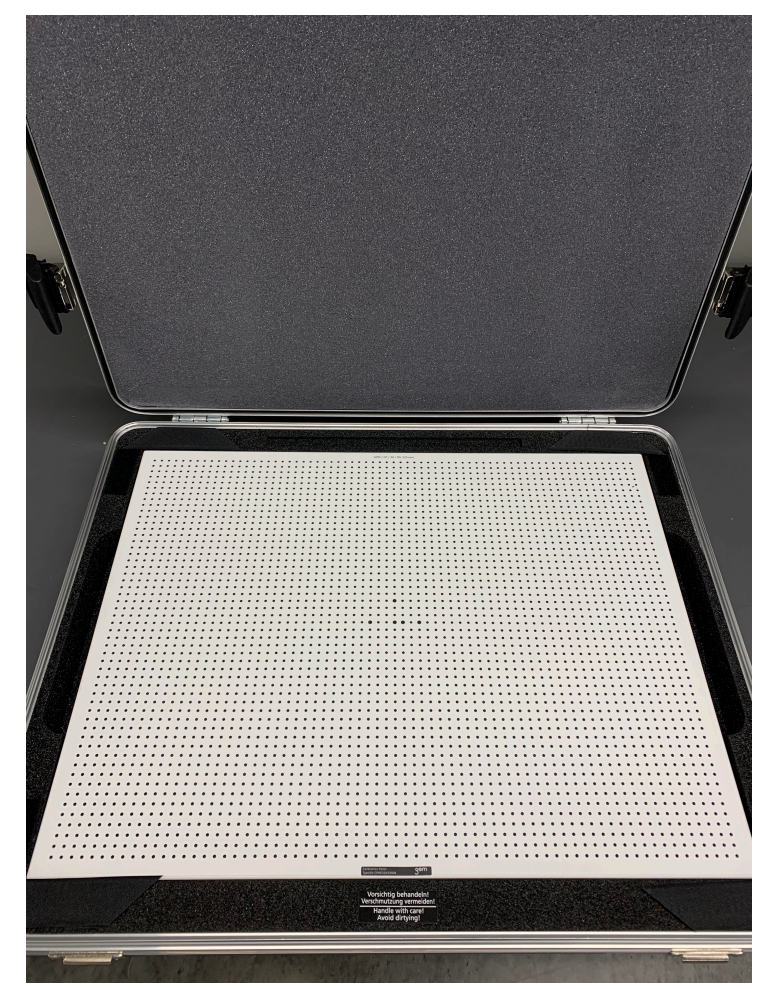

Figure 26: Three-dimensional DIC calibration board 


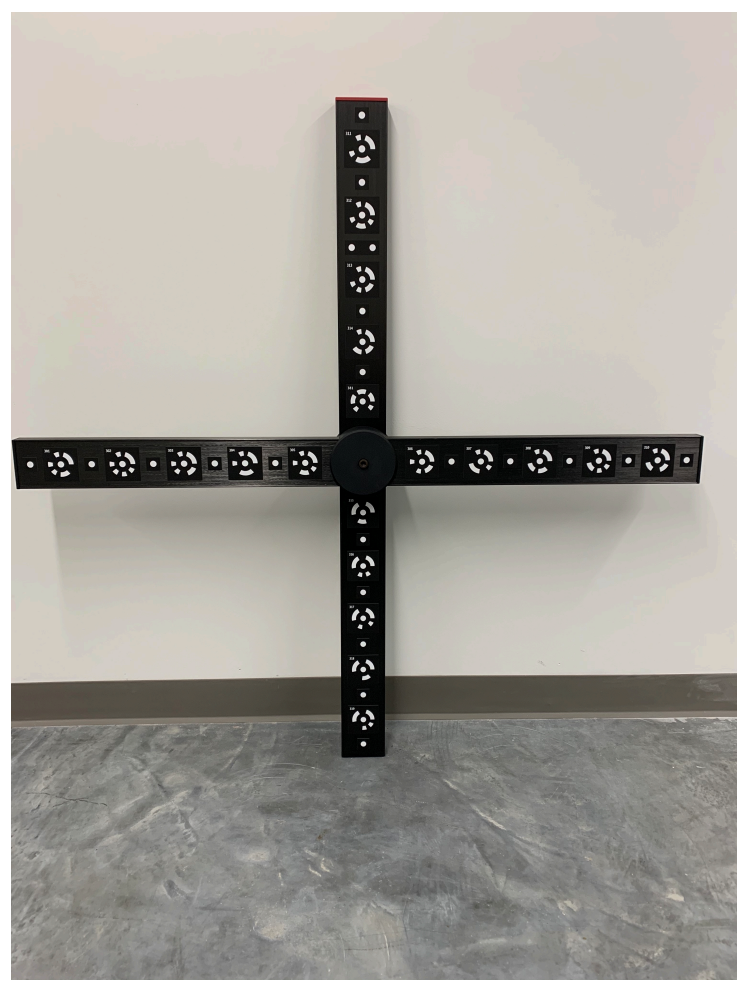

Figure 27: Three-dimensional DIC calibration cross

The geometric placement of the points on the calibration board and cross are measured to the micron level. A series of pictures of the calibration objects being moved and rotated through the measuring volume is used to calculate the position of the cameras in relation to each other.

\subsubsection{Uniaxial tensile testing}

In previous research performed by Cavallaro [11] and Smith [16], uniaxial tensile testing was performed in order to characterize drop-stitch panel constituent material properties. Rather than test each panel skin layer individually, this research aimed to obtain effective skin material properties from the drop-stitch panel skins as-fabricated; meaning the fabric and PVC layers were tested laminated together.

Samples were cut from the drop-stitch panel top and bottom PVC skins having a length of ten inches and a width of 1 inch. Samples were cut in the axial (warp), 
transverse (weft) and forty-five degree offset directions as shown below in Figure 28. This figure illustrates how the strips would be orientated on the drop-stitch panel normally.

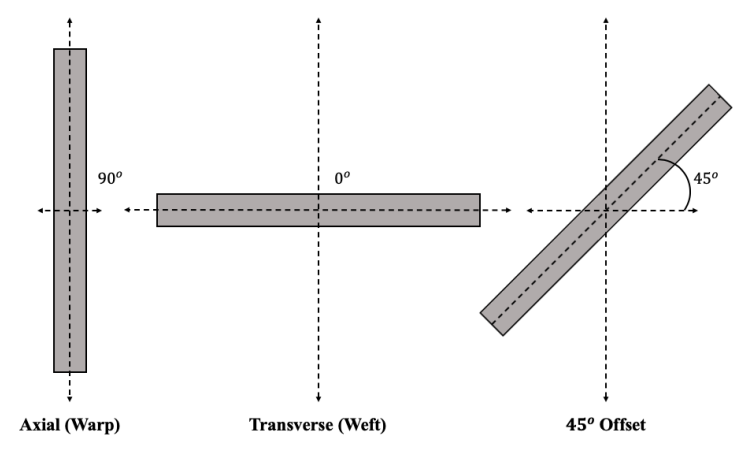

Figure 28: Uniaxial tensile testing samples

These samples were then loaded axially using one axis of a biaxial load frame. The samples were all initially placed in the machine grips with an eight inch gauge length, and pulled at a rate of half of an inch per minute until failure. The deformation was tracked using three dimensional digital image correlation as shown in Figure 29.

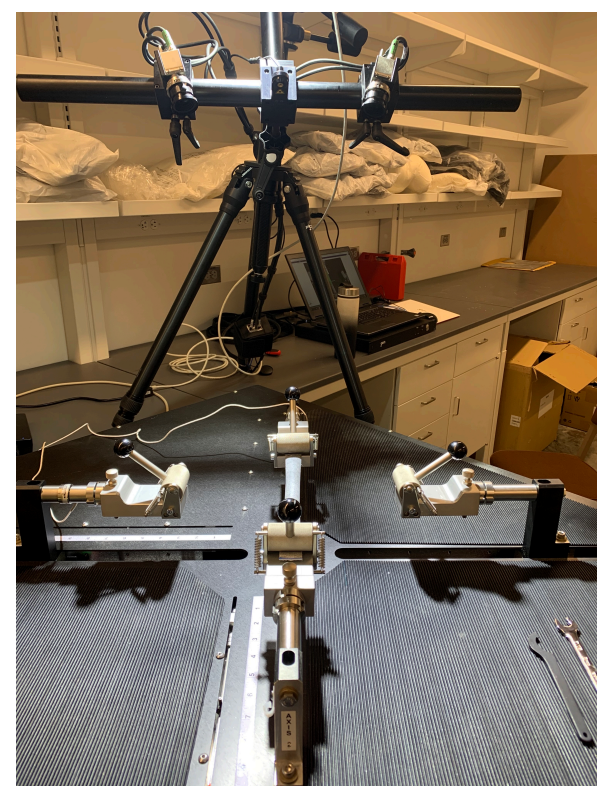

Figure 29: Uniaxial tensile testing experimental setup 
Figure 30 shows the region of interest and coordinate system used in the digital image correlation analysis.

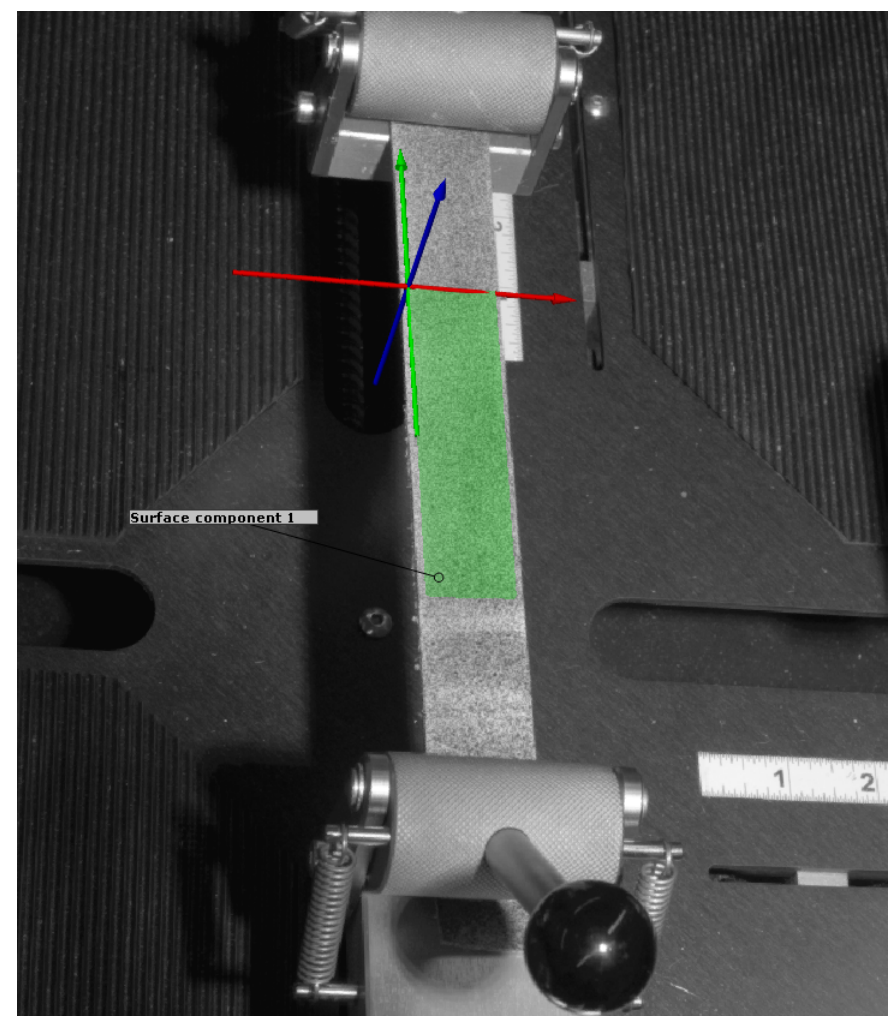

Figure 30: Uniaxial tensile testing DIC region of interest

The $\mathrm{x}$ direction was always taken to be the width of the sample, the y direction was always taken to be the length of sample and the $\mathrm{z}$ direction was then defined normal to the surface of the sample. Table 2 shows the directional notation used for the uniaxial tensile testing.

Table 2: Coordinate system of uniaxial tensile tests

\begin{tabular}{c|cc} 
Sample Direction & DIC X axis & DIC Y axis \\
\hline Axial (Warp) & Transversefibers & Axialfibers \\
Transverse (Weft) & Axialfibers & Transversefibers
\end{tabular}

The $45^{\circ}$ offset sample followed the same coordinate system orientation as the axial and transverse samples having the $\mathrm{x}$ axis be the width of the sample, the $\mathrm{y}$ 
axis be the length and the $\mathrm{z}$ axis be out of plane however both the $\mathrm{x}$ and $\mathrm{y}$ axis contained $45^{\circ}$ offset fibers from the axial and transverse directions, in addition to the $\mathrm{z}$ axis still being out of plane.

\subsubsection{Biaxial tensile testing}

Previous research has indicated that uniaxial testing isn't sufficient to characterize the drop-stitch panel skin material properties. Ideally biaxial tensile testing should be performed with a ratio of biaxial tension stresses matching the ratio of biaxial tension stresses produced in the actual structure from inflation. This ratio is given by equation 29 [11].

$$
N_{\text {ratio }}=\frac{\sigma_{\text {Transverse }}}{\sigma_{\text {Axial }}}
$$

Table 3 shows the biaxial inflation stresses and corresponding biaxial stress ratio for the drop-stitch panel used in this research.

Table 3: Panel inflation stress ratio

\begin{tabular}{c|ccc} 
Inflation pressure (psi) & Axial stress (psi) & Transverse stress (psi) & $\mathbf{N}_{\text {ratio }}$ \\
\hline $\mathbf{5}$ & 171 & 194 & 1.1 \\
$\mathbf{1 0}$ & 342 & 389 & 1.1 \\
$\mathbf{1 5}$ & 513 & 584 & 1.1
\end{tabular}

To achieve this biaxial stress ratio in a tensile test a cruciform sample was used. Each arm had a width of 1.875 inches. Each axis of the biaxial load frame was programmed to pull the sample individually. Both axis 1 and axis 2 began with a five inch gauge length. Axis 1 loaded the warp (axial) direction of the sample at a rate of $100 \mathrm{lbs} / \mathrm{min}$. Axis 2 loaded the weft (transverse) direction of the sample at a rate of $110 \mathrm{lbs} / \mathrm{min}$. Figure 31 illustrates how the cruciform sample would be orientated on the drop-stitch panel. 


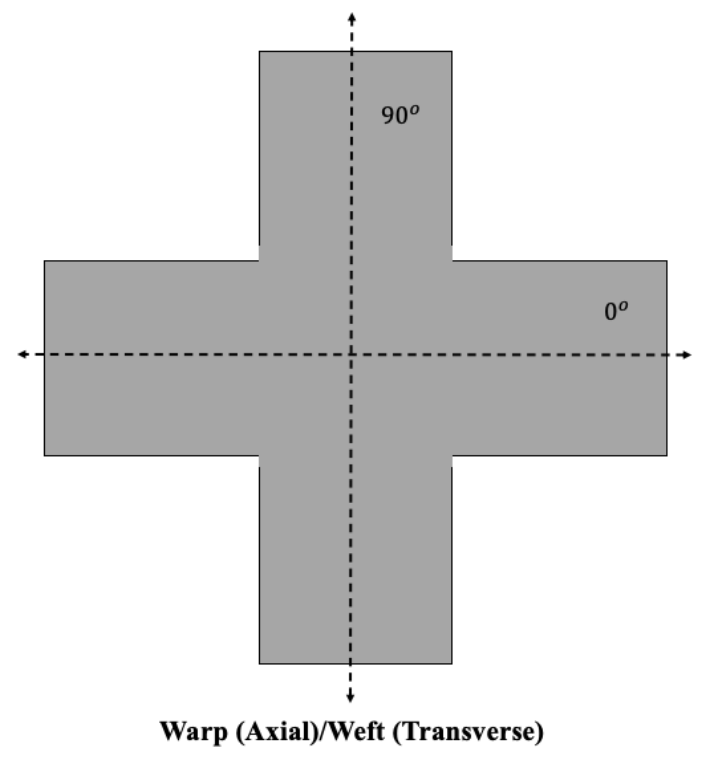

Figure 31: Biaxial tensile cruciform

The testing was conducted to failure and analyzed using digital image correlation as shown in Figure 32.

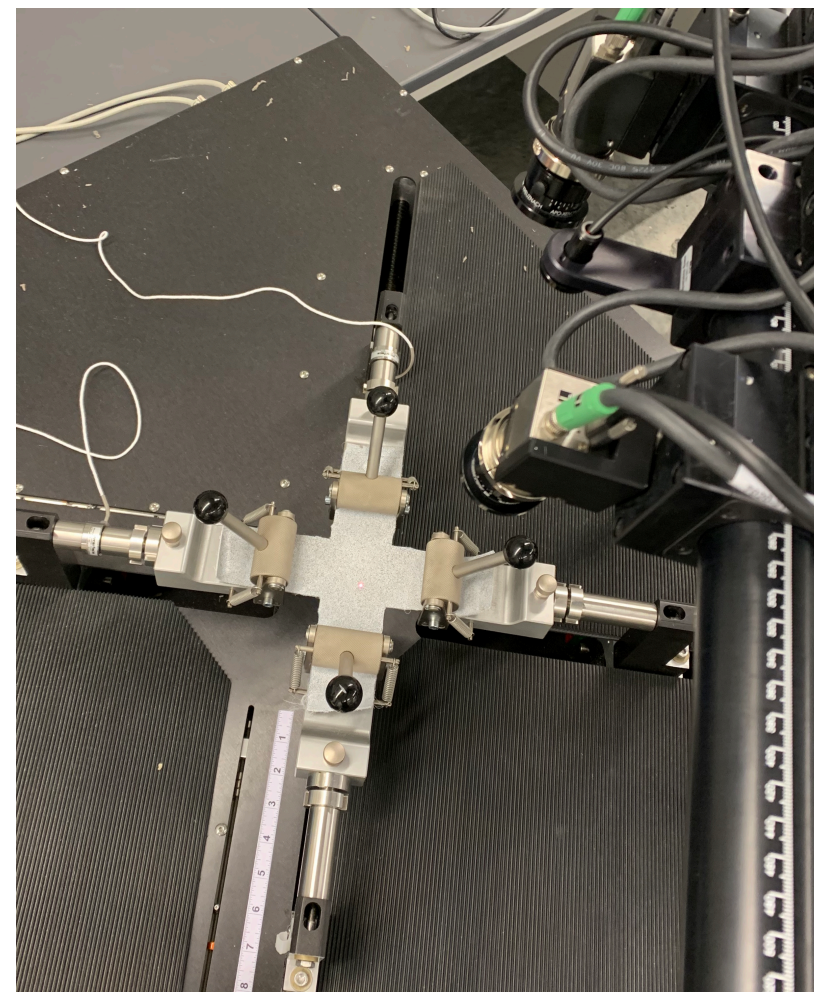

Figure 32: Biaxial tensile testing experimental setup 
Figure 33 shows the region of interest and coordinate system used in the biaxial tensile testing. The $\mathrm{x}$ direction was defined to be axis 2 which housed the transverse (weft) direction of the sample, while the y direction was defined to be axis 1 which housed the axial (warp) direction of the sample. The $\mathrm{z}$ direction was defined to normal to the sample. Thus, strain in the $\mathrm{x}$ direction represented transverse strain while strain in the y direction represented axial strain in the digital image correation software.

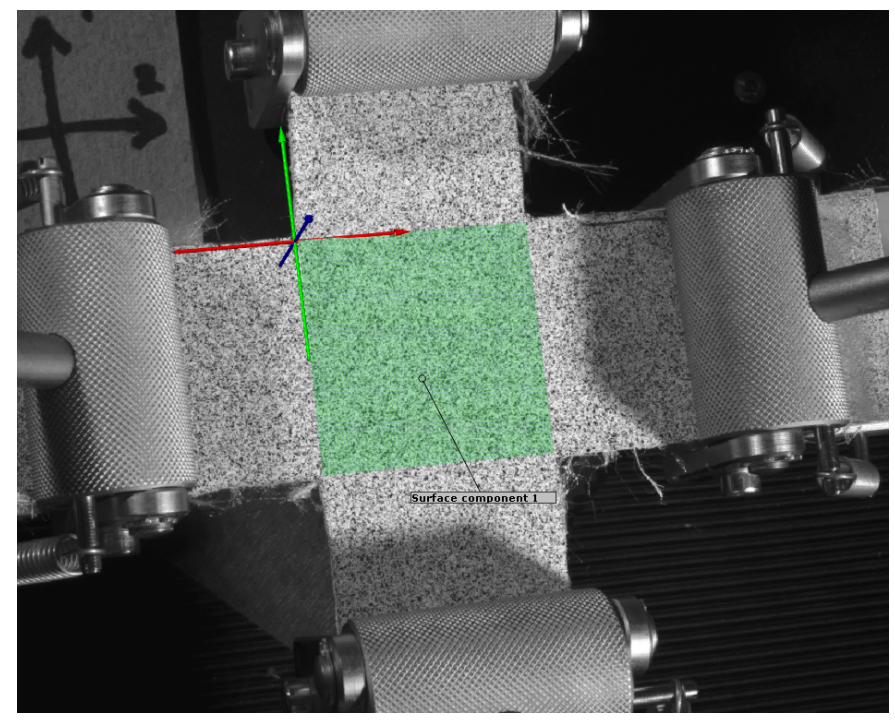

Figure 33: Biaxial tensile testing DIC region of interest

\subsubsection{Inflation testing}

In addition to replicating the biaxial inflation stresses in the drop-stitch panel skin using a biaxial load frame, a digital image correlation analysis of a panel inflation was performed on a local region of the panel skin. Figure 34 shows the placement of the local region on the drop-stitch panel. 

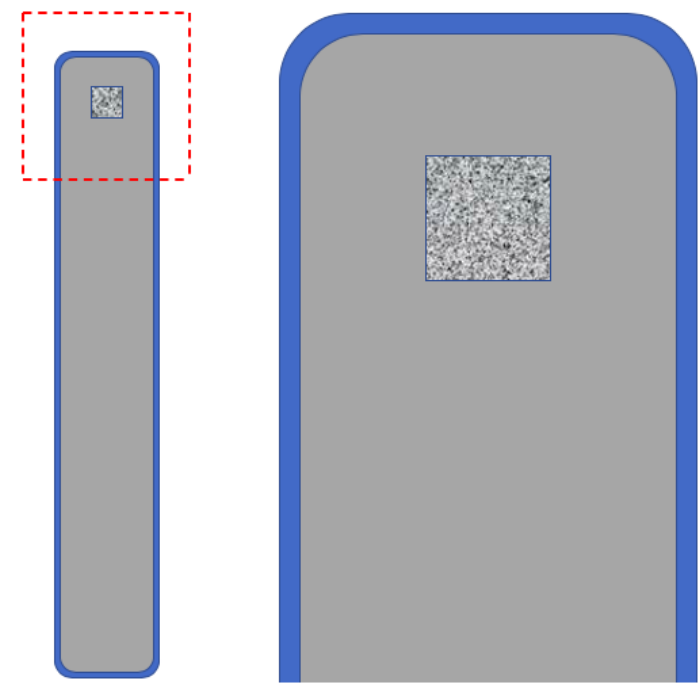

Figure 34: Inflation testing local region

The panel was inflated incrementally from 0.5 psi to 15 psi in 0.5 psi increments. As shown in figure 35, the $\mathrm{x}$ axis was defined to be the transverse direction of the panel, the y axis was defined to be the axial direction of the panel and the $\mathrm{z}$ axis was defined to be normal to the panel.

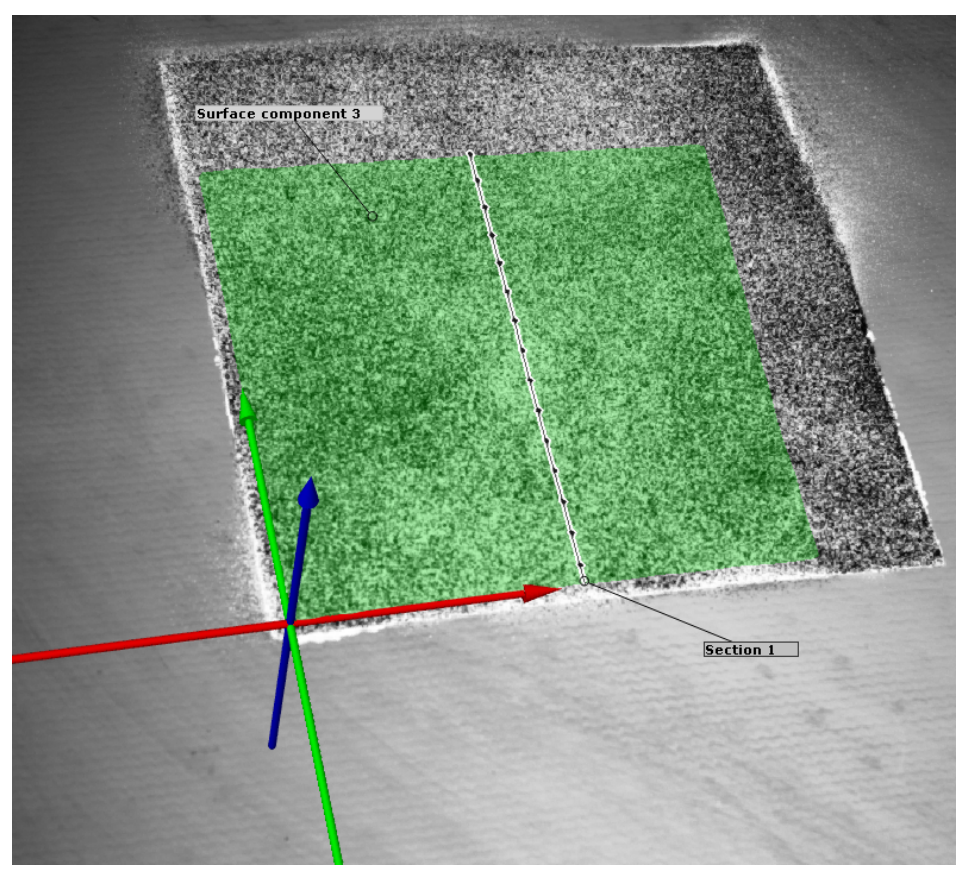

Figure 35: Inflation testing DIC region of interest 
Thus, strains in the $\mathrm{x}$ direction were transverse inflation strains and strains in the y direction were axial inflation strains.

\subsubsection{Four-point bend testing}

A common test of the behaviour of drop-stitch inflatable panels under loading conducted in previous research are four-point bending tests at various inflation pressures. Increasing the the inflation pressure under the bending load produces a a stiffer structure which is reflected in the load vs de

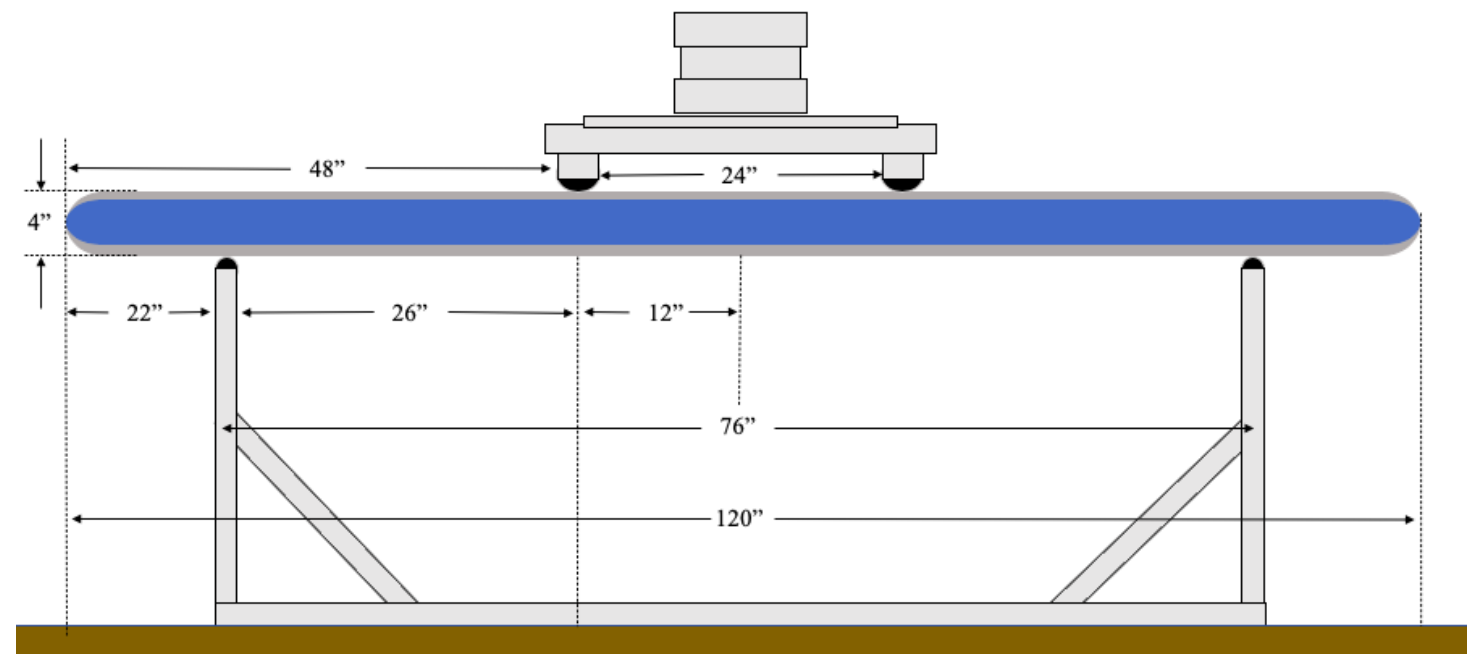

Figure 36: Four-point bend test diagram

Figure 36 shows the four-point loading configuration for the bend testing performed in this research. As shown, the panel was supported at a span of 76 inches and and contacted by two loading pins 12 inches from the panel midpoint. Once the loading pins were manually lowered to barely touch the top surface of the panel, the load frame was programmed to deflect the panel 3 inches at a rate of 0.5 inches per minute as shown in Figure 37. The applied load was recorded throughout the entire deflection. Tests were conducted at inflation pressures of 5,10 , and 15 psi. 


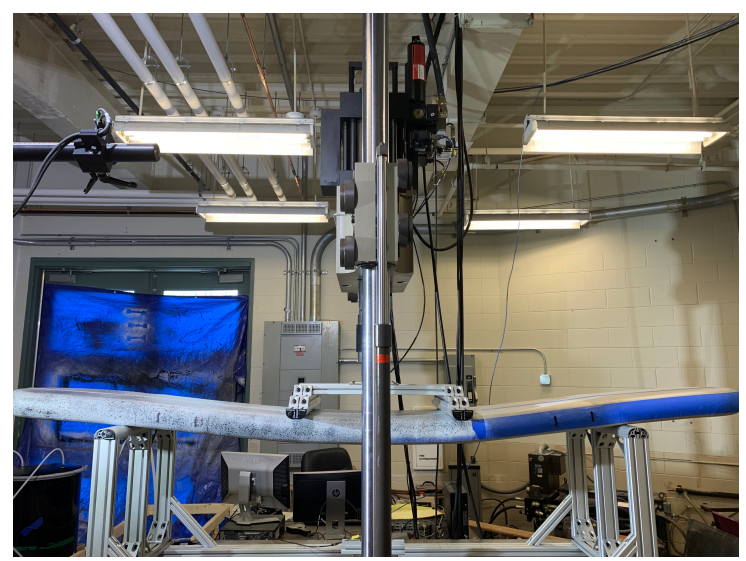

Figure 37: Four-point bend test 3 inch deflection

Using a larger field of view, digital image correlation was used to analyze both the panel top surface displacement field and strains of a section of the panel ranging from the left end of the panel to the left loading pin. Figure 38 shows the digital image correlation camera setup.

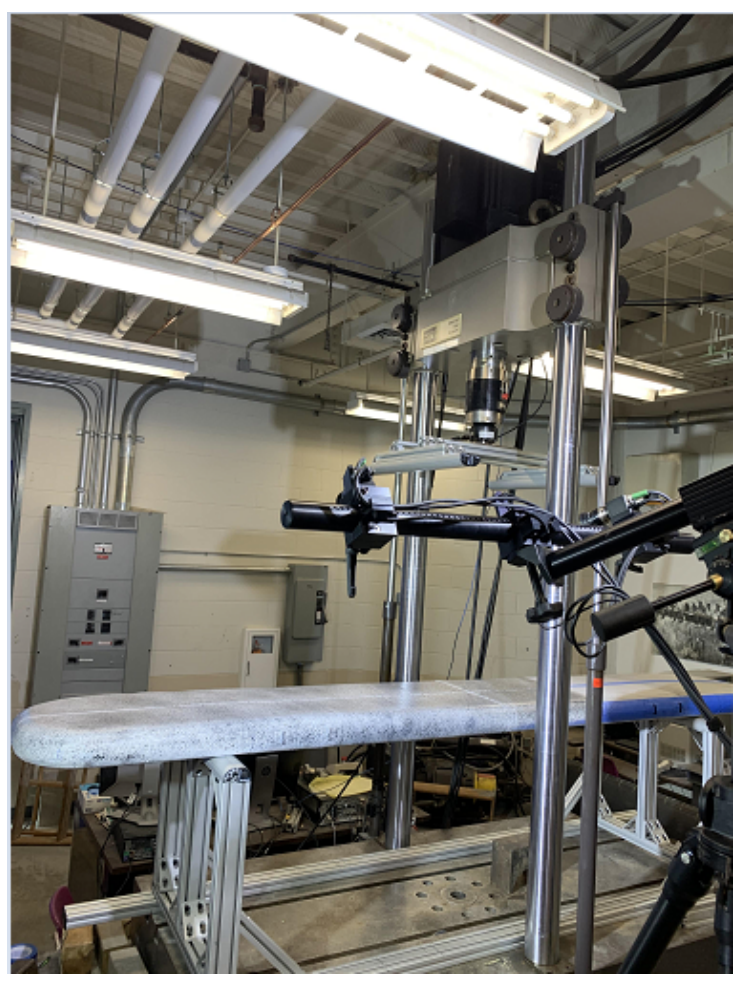

Figure 38: Four-point bend test DIC camera view of panel top

Figure 39 shows the region of interest and coordinate system used in analyzing 
the panel's top surface during four-point bend testing. The x direction was defined to be the transverse direction of the panel, the y direction was defined to be the axial direction of the panel and the $\mathrm{z}$ direction was defined normal to the panel's top surface.

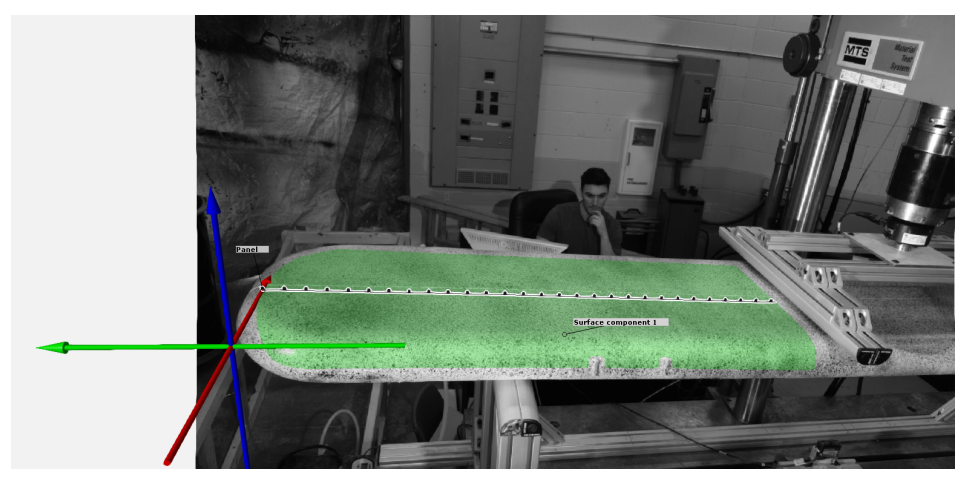

Figure 39: Four-point bend test panel top DIC region of interest

A series of bend tests was conducted with an identical loading configuration and inflation pressures of 5, 10 and 15 psi in order to also analyze the panel bottom using digital image correlation. Figure 40 shows the digital image correlation cameras setup to capture an image series of the deflection of the bottom surface of the panel.

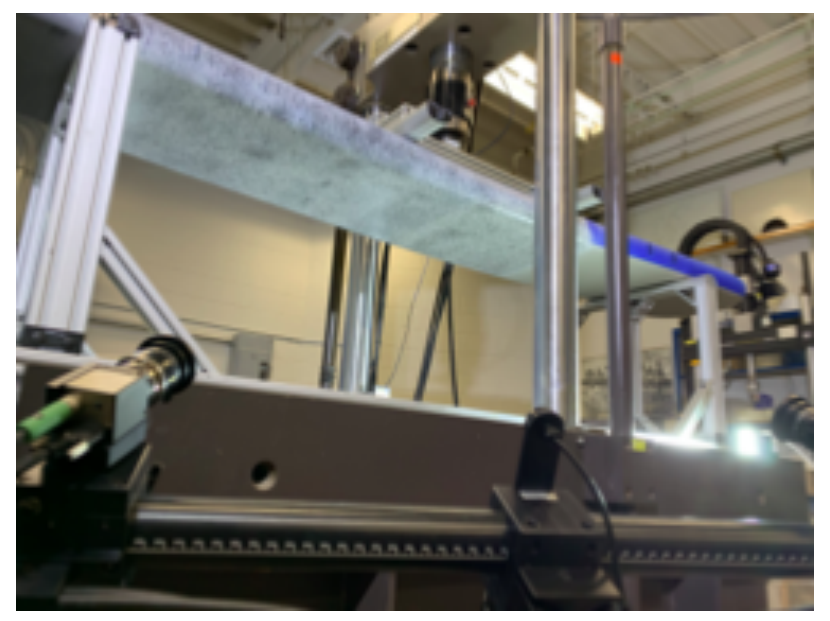

Figure 40: Four-point bend test DIC camera view of panel bottom

The region of interest of the panel bottom digital image correlation analysis 
spanned from the midpoint to just before the left support, including the panel region under the left loading pin. Like the panel top surface region of interest, the $\mathrm{x}$ axis was defined to be the transverse direction of the panel, the $\mathrm{y}$ axis was defined to be the axial direction of the panel and the $\mathrm{z}$ direction was defined to be normal to panel bottom surface.

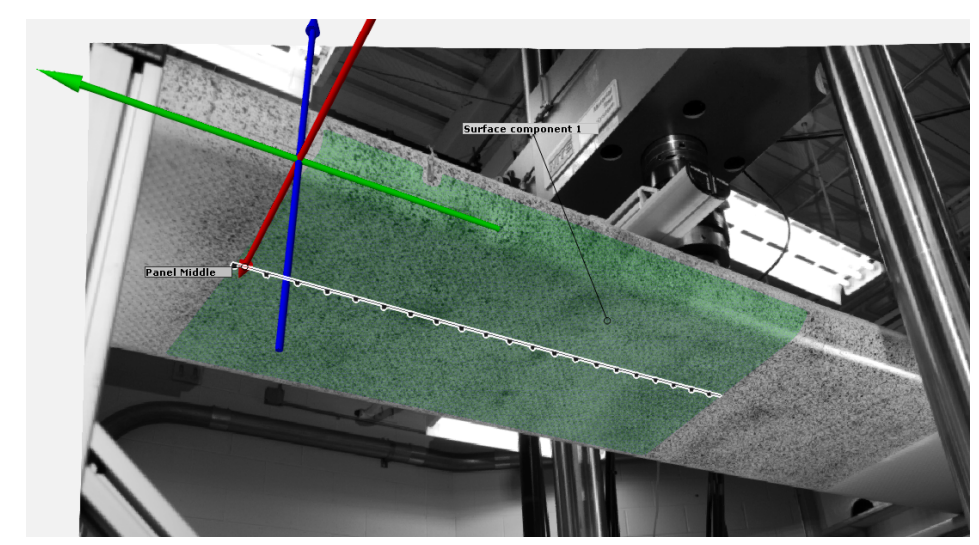

Figure 41: Four-point bend test panel bottom DIC region of interest

Similar to measurements on the panel top surface, the highlighted white section of the region of interest was used to analyze displacement fields and strains along the bottom profile of the panel as a function of position. 


\section{CHAPTER 4}

\section{Findings}

\subsection{Uniaxial tensile testing}

Figure 42 shows the load vs position plots of uniaxial tensioned warp and weft skin samples. In these plots, the position is measured by the load frame actuator displacement and the load is measured by the load frame load cell.

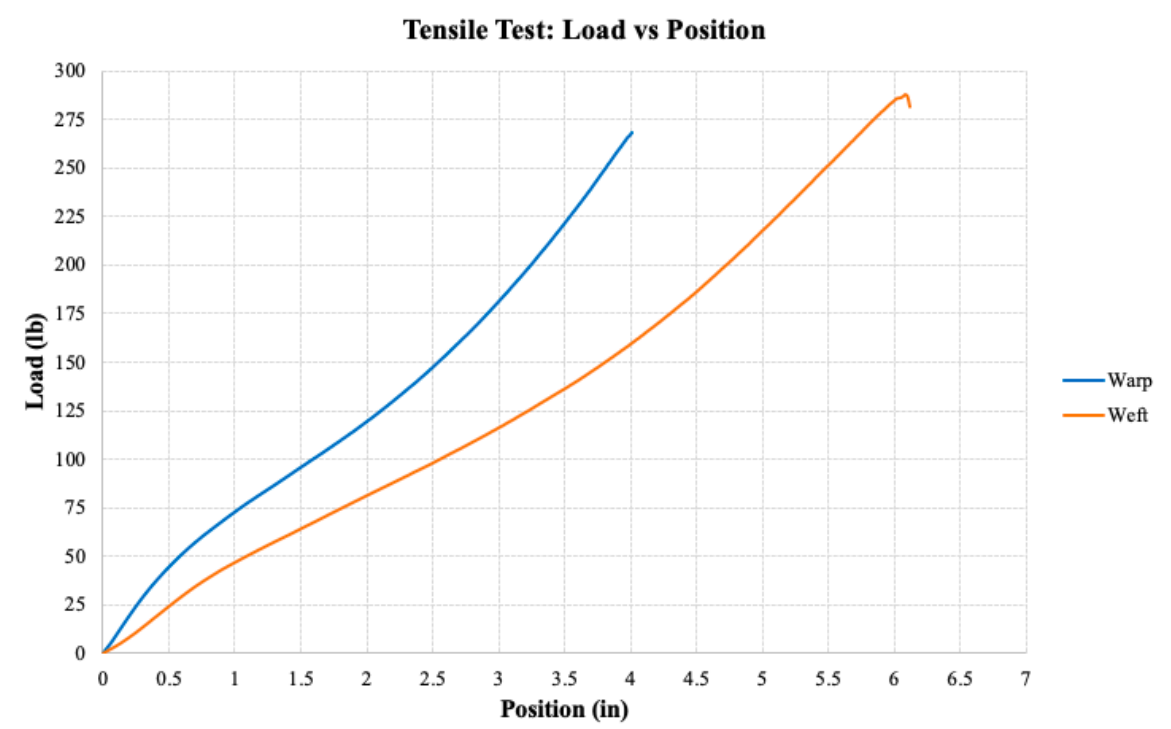

Figure 42: Uniaxial tension warp and weft load vs position

The loads recorded were converted to stresses and these stresses were plotted against strain values measured from digital image correlation.

Figures 43 through 46 show the axial and transverse strain fields of the warp and weft samples from digital image correlation. The histogram on the right shows the density of the the strain values present in the figure. The specific magnitude of the strains present is given by the strain percentage values corresponding with the colors next to the histogram. Figure 43 shows the strain concentration associated with the cut drop stitch yarns. It is evident from this result that drop stitch yarns emanate from the yarns oriented in the warp direction. 

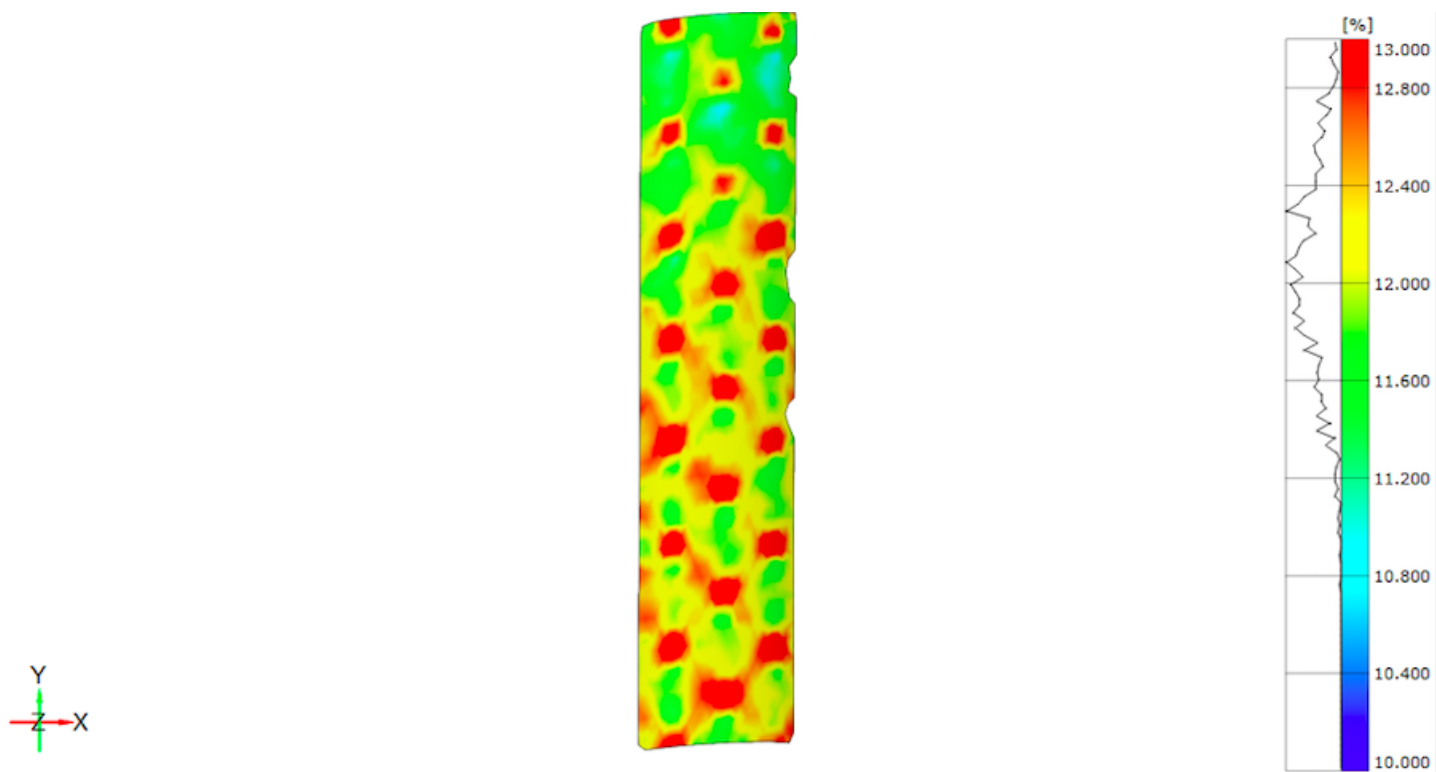

Figure 43: Uniaxial tension warp sample axial strain field
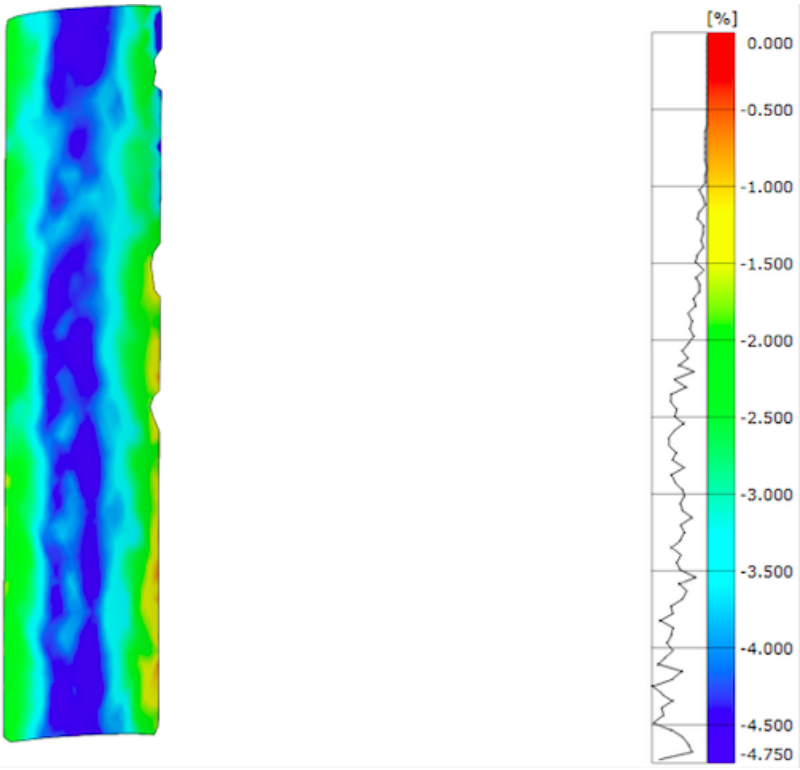

Figure 44: Uniaxial tension warp sample transverse strain field 

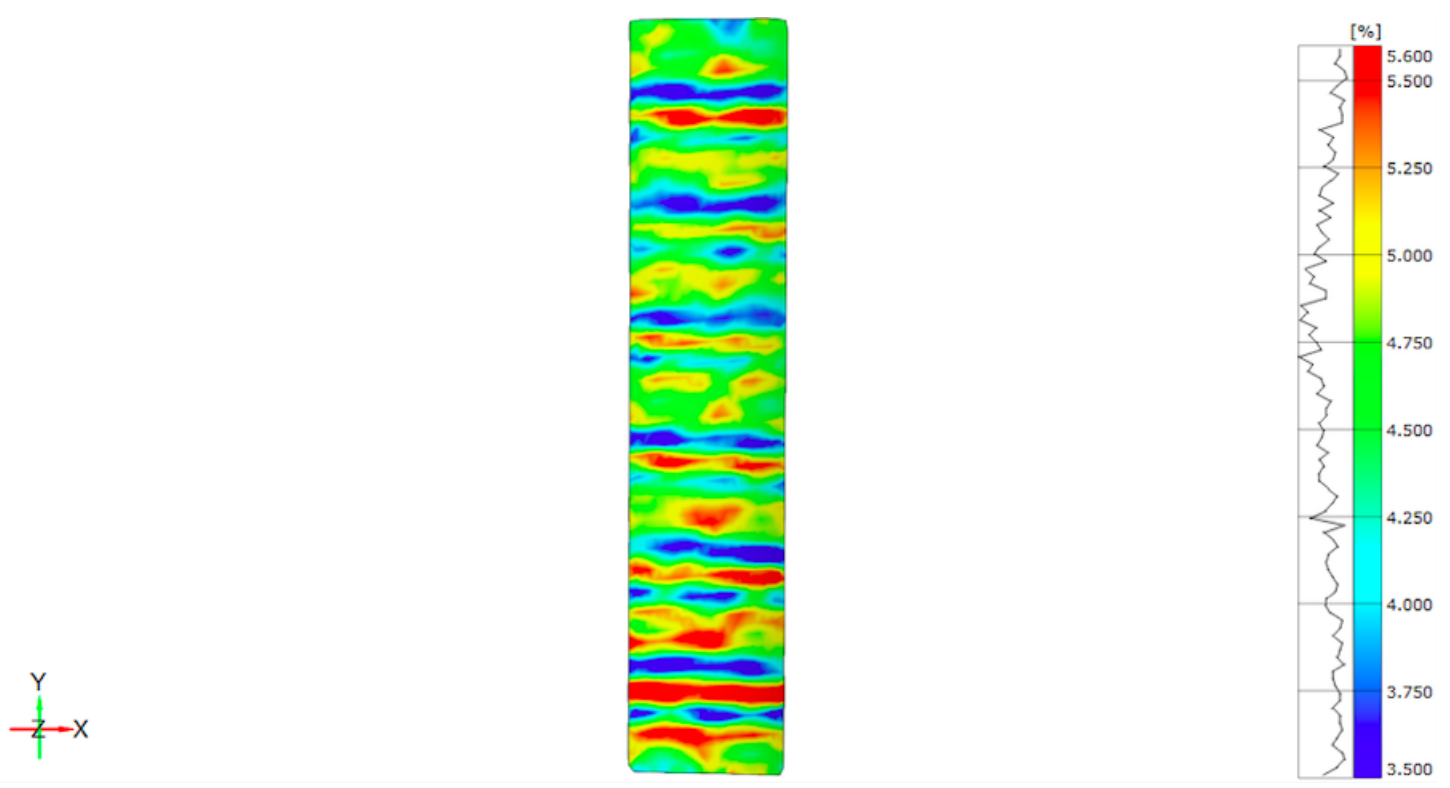

Figure 45: Uniaxial tension weft sample axial strain field

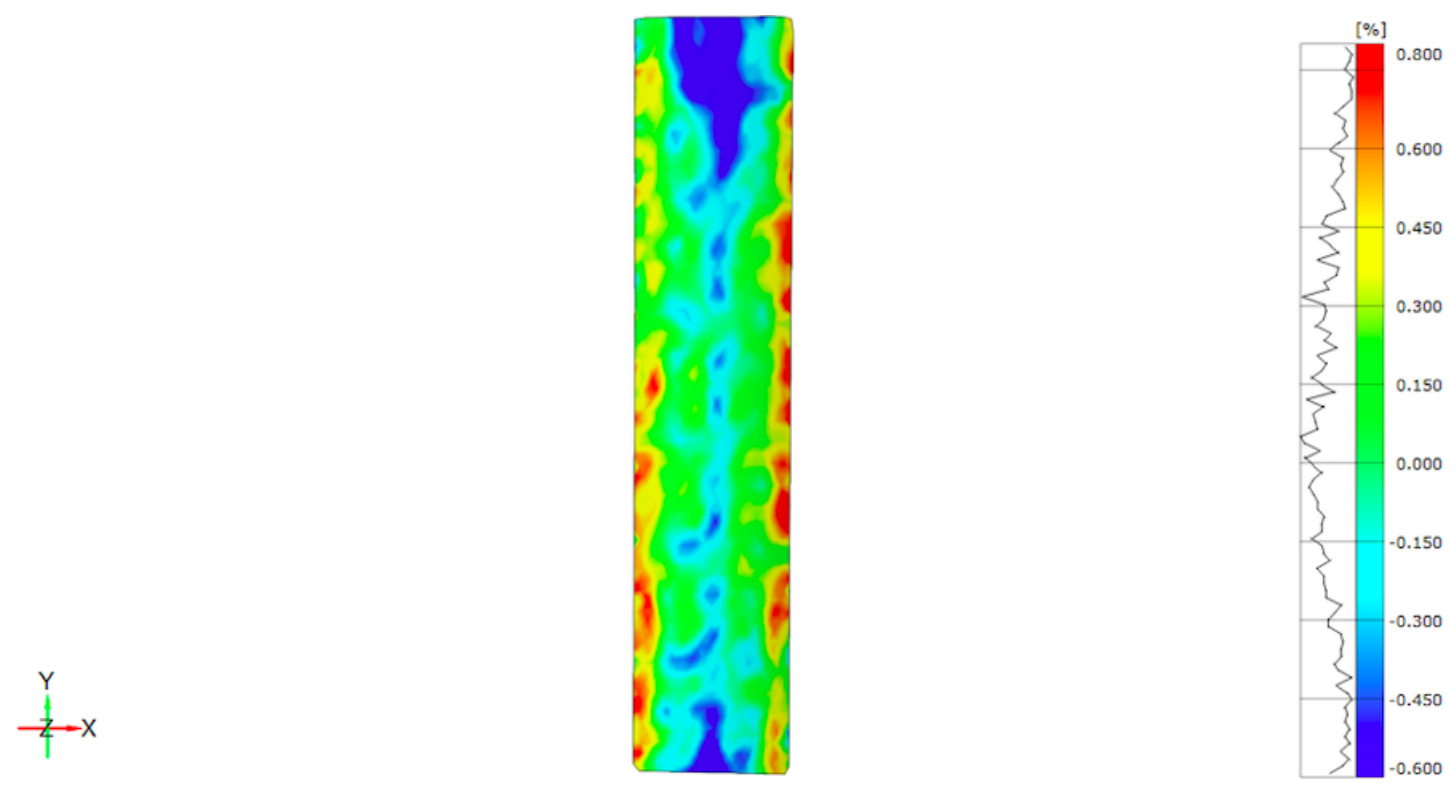

Figure 46: Uniaxial tension weft sample transverse strain field

Figure 47 shows the stress vs strain curves of both warp and weft skin samples. Stresses were calculated from the measured applied tensile load and strains were averaged from a central section of the digital image correlation strain field. 


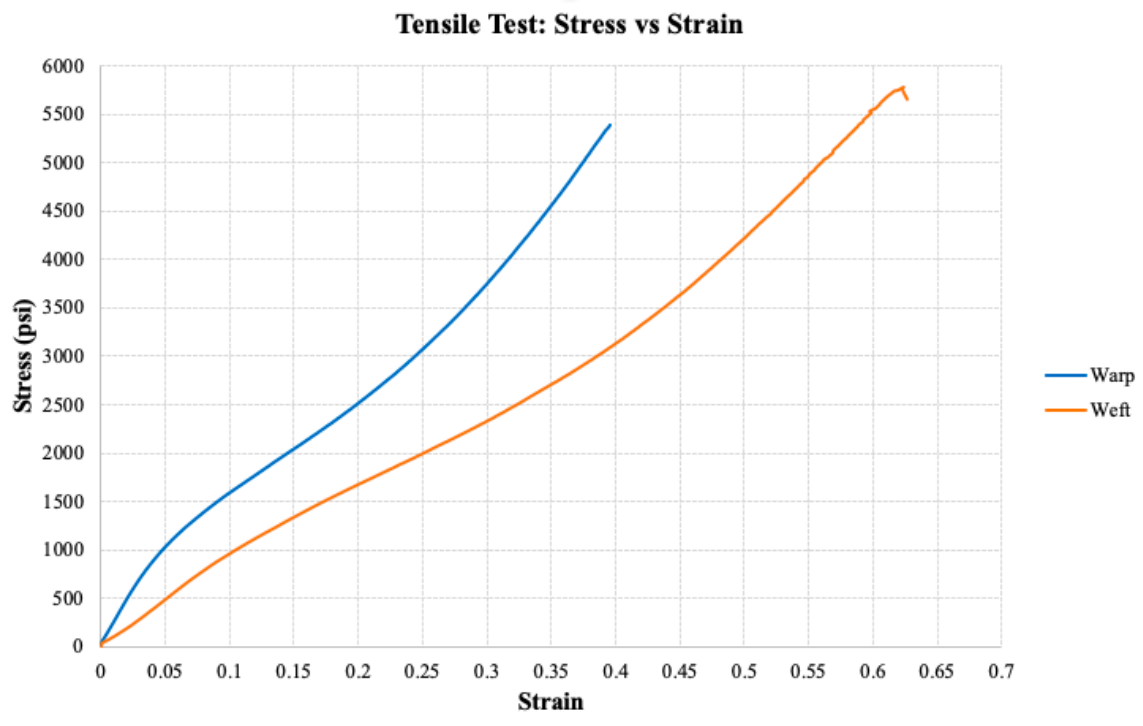

Figure 47: Uniaxial tension warp and weft stress vs strain

Table 4: Experimentally determined elastic moduli from uniaxial loading

\begin{tabular}{c|c} 
Direction & Elastic Modulus (psi) \\
\hline Warp & 20,451 \\
Weft & 9,770
\end{tabular}

Table 4 gives the elastic moduli of the panel skin. These moduli are the initial slopes of the uniaxial stress strain curves in figure 47.

Previous research has indicated that uniaxial tensile tests are not sufficient to to fully characterize the behaviour of the drop-stitch inflatable panel skin. Since inflated panel skins are subjected to biaxial loading, it is expected that biaxial tensile tests are expected to provide an improved simulation of in-service load conditions. Figure 47 shows the uniaxial response for loading in the warp and weft directions and will be used for comparison purposes later in the chapter.

Uniaxial tensile testing also provides a measure of Poisson's ratio. Figure 48 shows the DIC measured negative transverse strains plotted against axial strains measured during uniaxial tension in the warp direction, and Figure 49 shows the 
DIC measured negative transverse strains plotted against axial strains measured during uniaxial tension in the weft direction. The slopes of these curves can be interpreted as the Poisson ratio's $\nu_{12}$ and $\nu_{21}$, respectively, and are tabulated in Table 5.

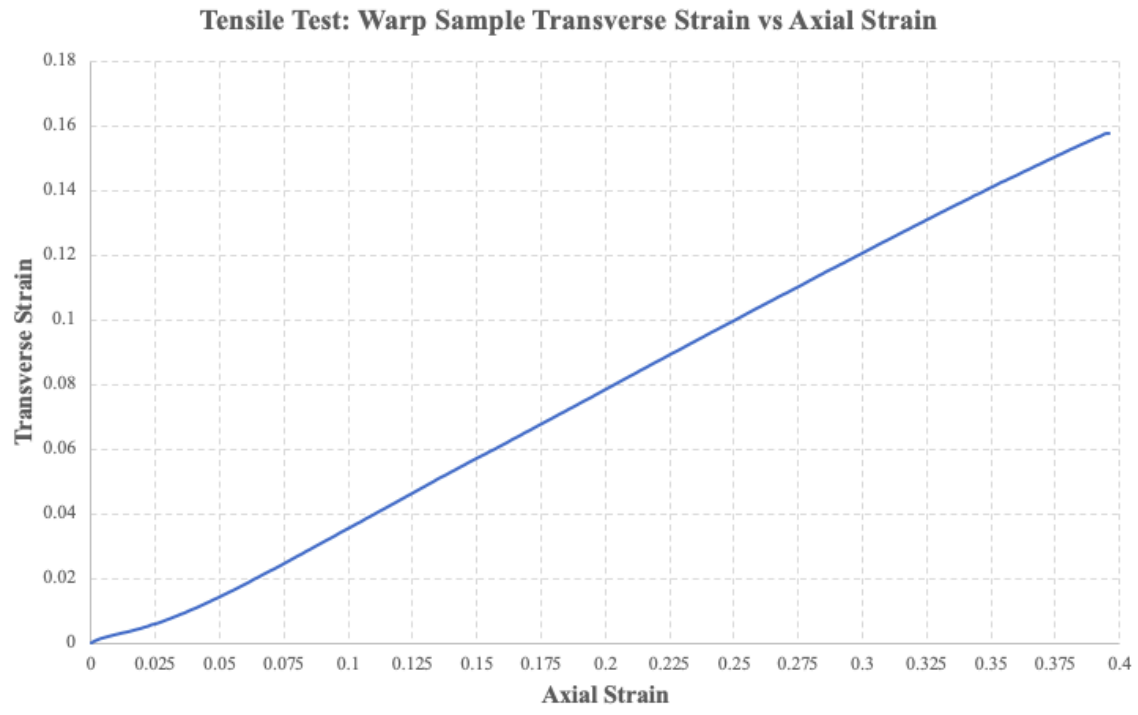

Figure 48: Uniaxial tension warp Poisson's ratio

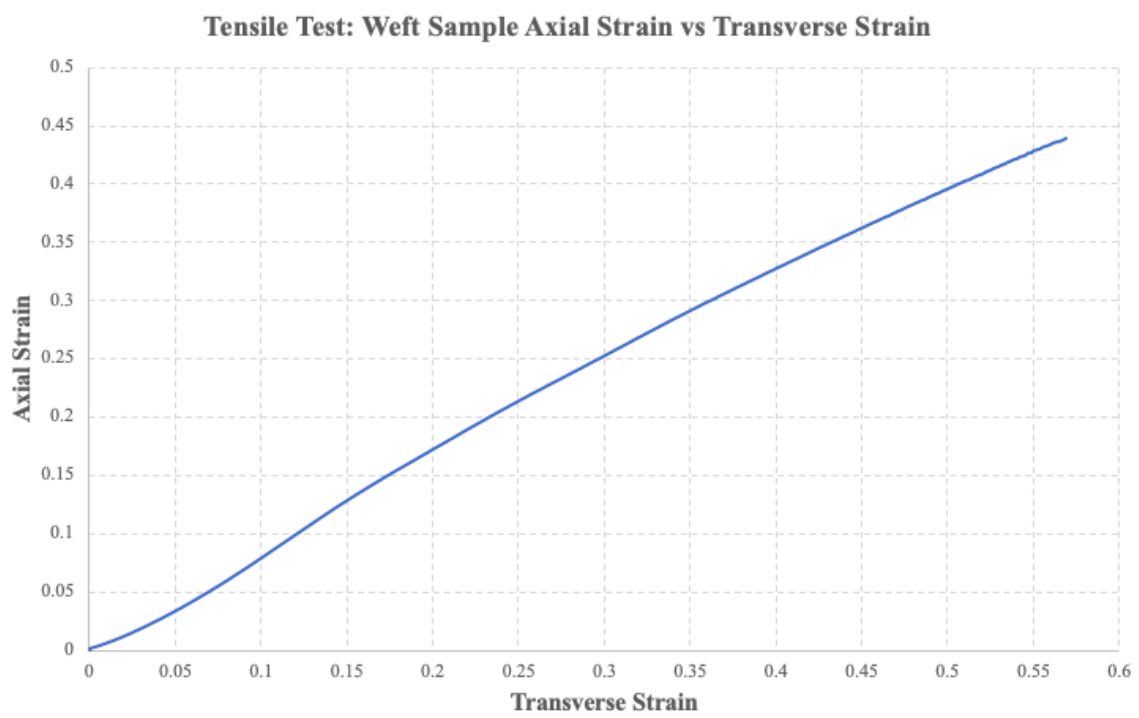

Figure 49: Uniaxial tension weft Poisson's ratio 
Table 5: Experimentally determined Poisson's ratios from uniaxial loading

\begin{tabular}{c|c} 
Direction & Poisson's Ratio \\
\hline Warp & $\nu_{12}=0.4211$ \\
Weft & $\nu_{21}=0.7873$
\end{tabular}

Figures 50 and 51 show the axial and transverse strain fields respectively of the $45^{\circ}$ offset panel skin from digital image correlation. Both figures show the offset strain concentration aligned with the fibers as compared to the normally oriented warp and weft skins.
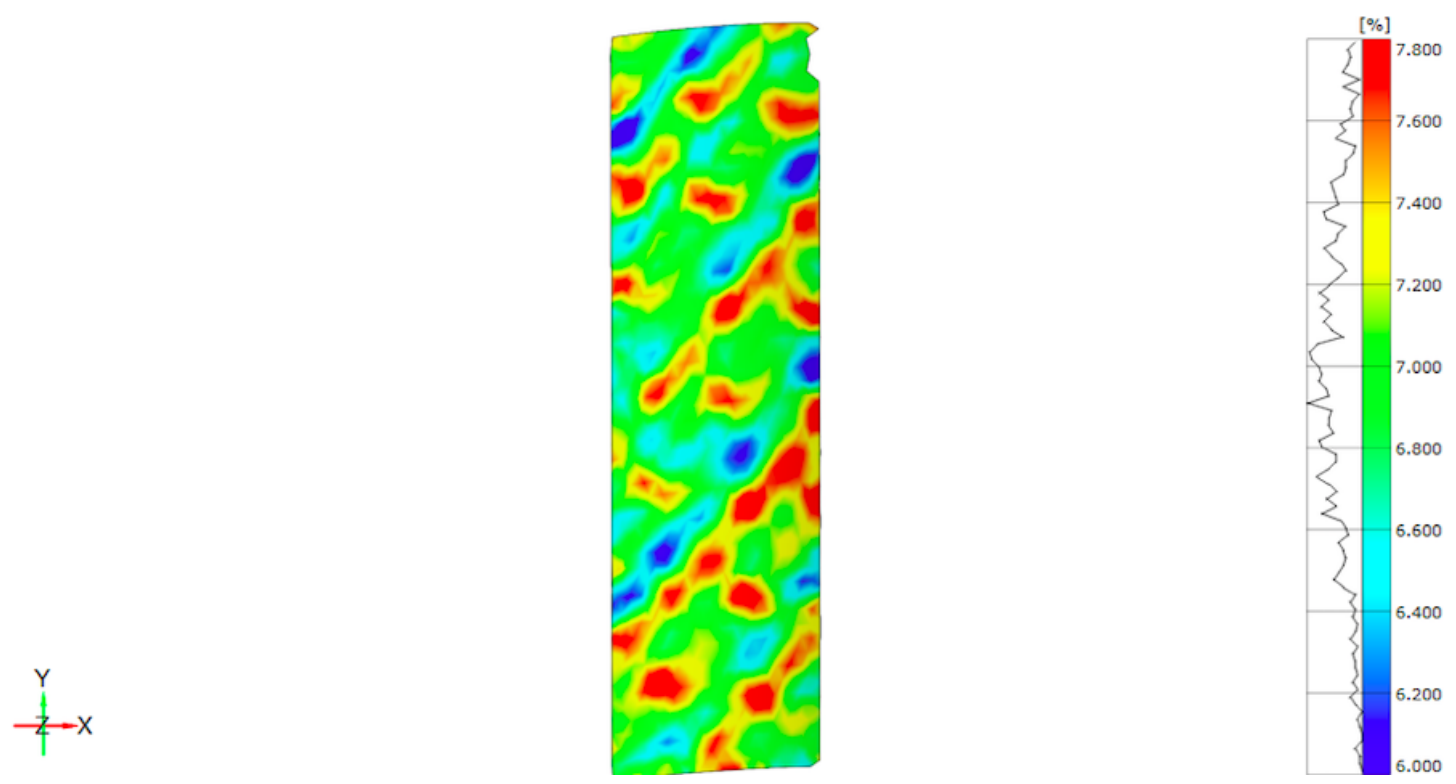

Figure 50: Uniaxial tension $45^{\circ}$ offset sample axial strain field 


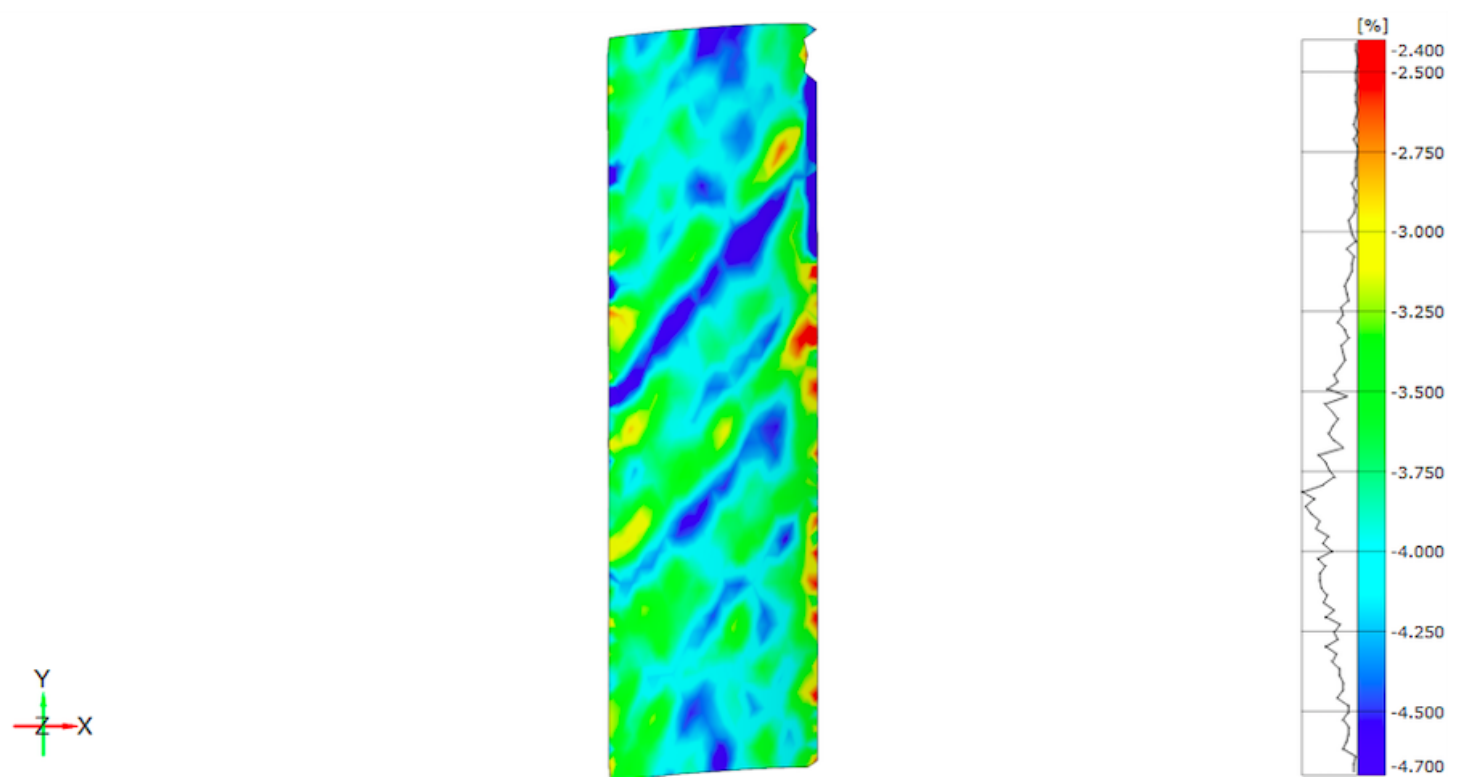

Figure 51: Uniaxial tension $45^{\circ}$ offset sample transverse strain field

Figure 52 shows the results of uniaxial tensile testing of a $45^{\circ}$ offset skin sample. Shear stress was calculated from the applied load while shear strains were from calculated from measured digital image correlation values.

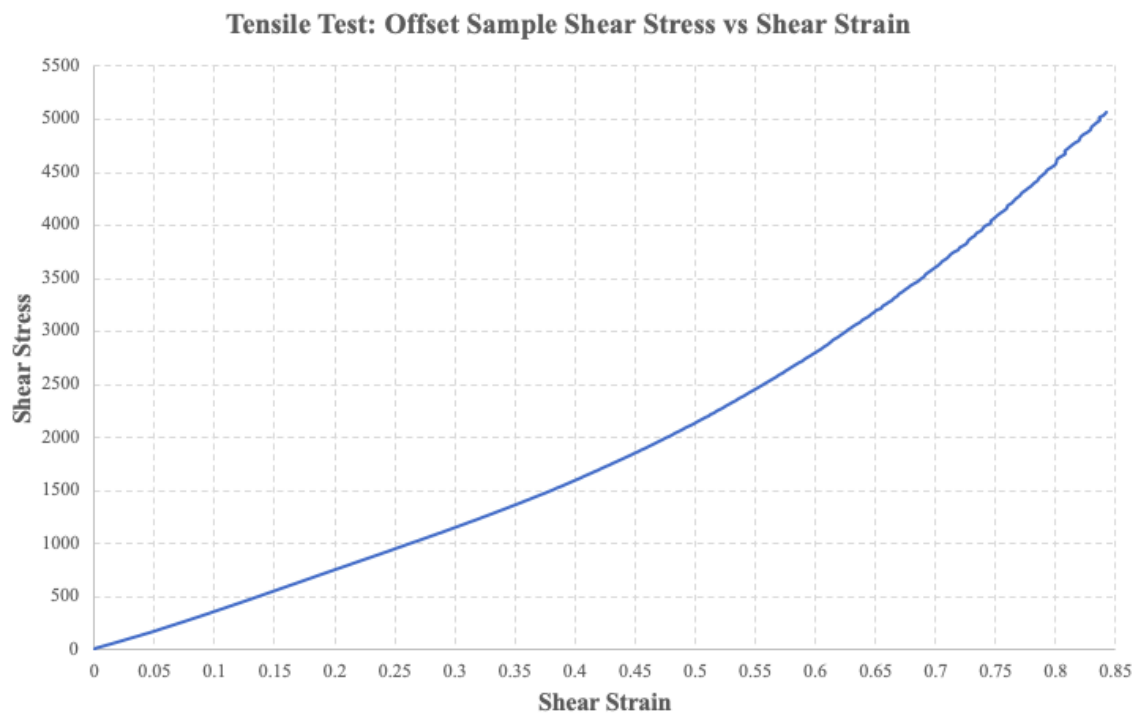

Figure 52: Uniaxial tension $45^{\circ}$ offset shear stress vs shear strain

Using the procedure described in section 3.1, equation 14 was used to calculate the shear modulus of the drop-stitch panel skin. Based on the initial slope of the 
shear stress-shear strain curve, the shear modulus is estimated to be $G=1,885$ psi.

\subsection{Biaxial tensile testing}

Figure 53 shows the load vs position curves of a normally oriented cruciform skin sample, and the uniaxial load vs position curves from 42. The solid lines represent the cruciform tested in the biaxial tensile test, while the dotted lines represent the individual uniaxial tensile tests of the warp and weft skin samples.

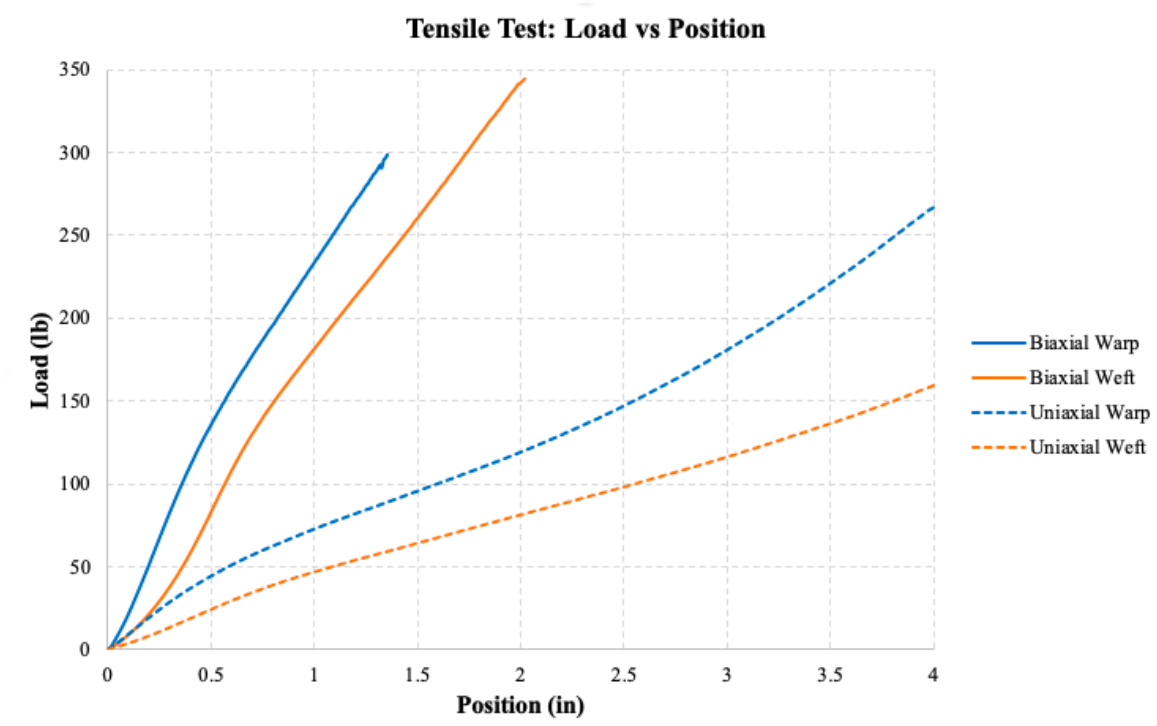

Figure 53: Biaxial and uniaxial tension load vs position

The measured loads applied to the cruciform skin sample were converted to stresses and plotted against average strains measured from digital image correlation.

Figures 54 through 56 show the axial strain fields measured by digital image correlation when the applied loads from the biaxial load frame are equivalents of 5, 10 and 15 psi inflation stresses. 


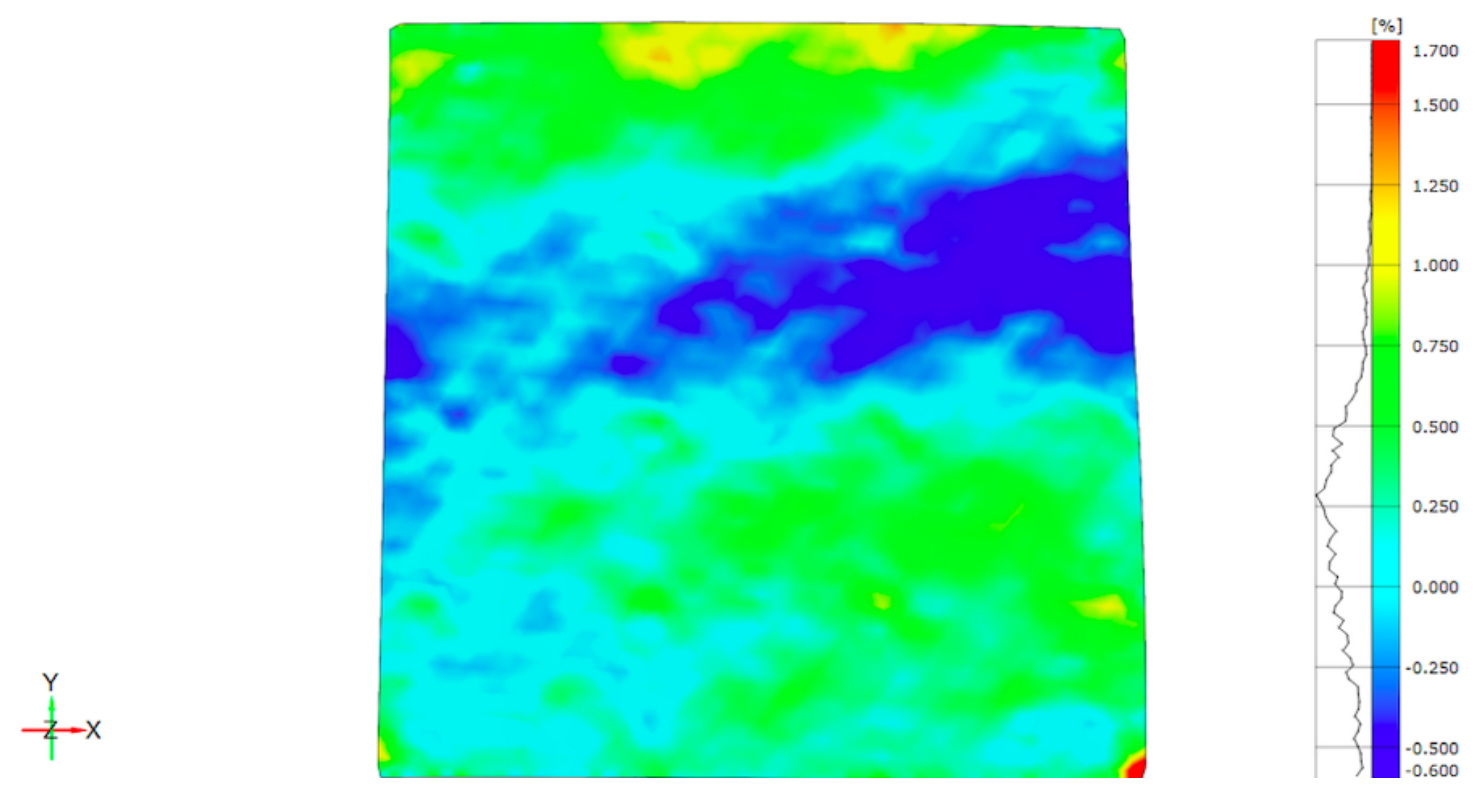

Figure 54: Biaxial tension state of 5 psi inflation pressure axial strain field

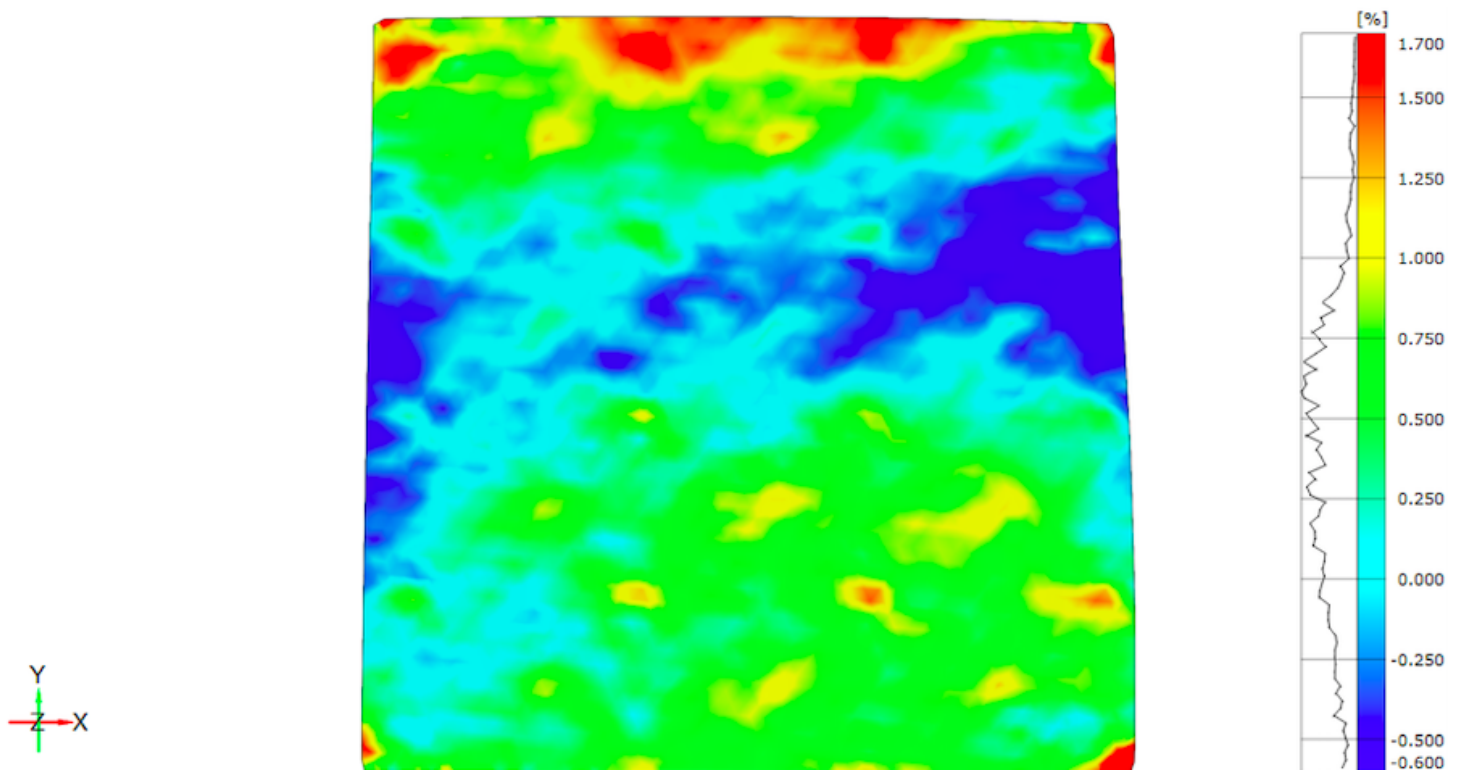

Figure 55: Biaxial tension state of 10 psi inflation pressure axial strain field 

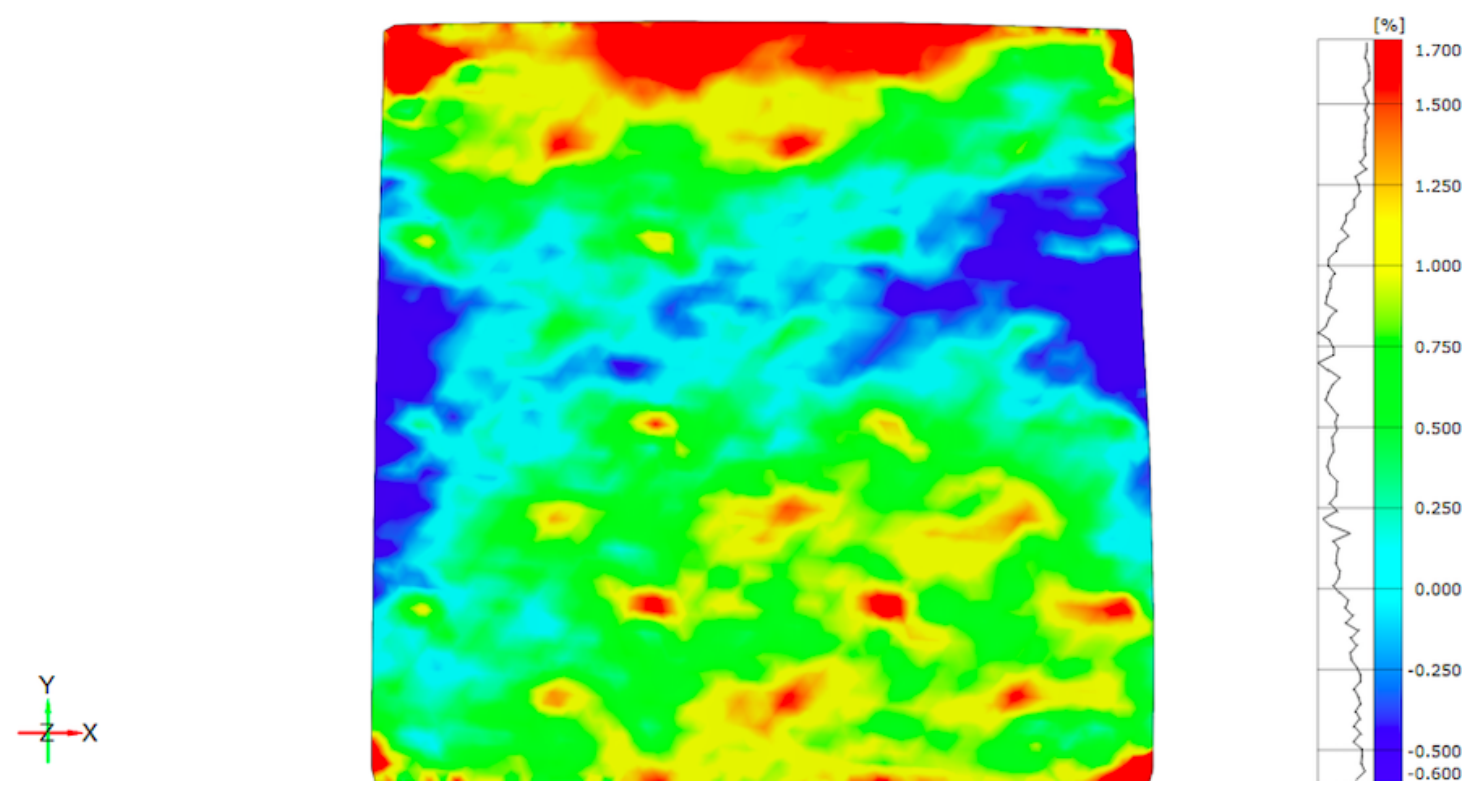

Figure 56: Biaxial tension state of 15 psi inflation pressure axial strain field

Figures 57 through 59 show the transverse strain fields measured by digital image correlation when the applied loads from the biaxial load frame are equivalents of 5, 10 and 15 psi inflation stresses.

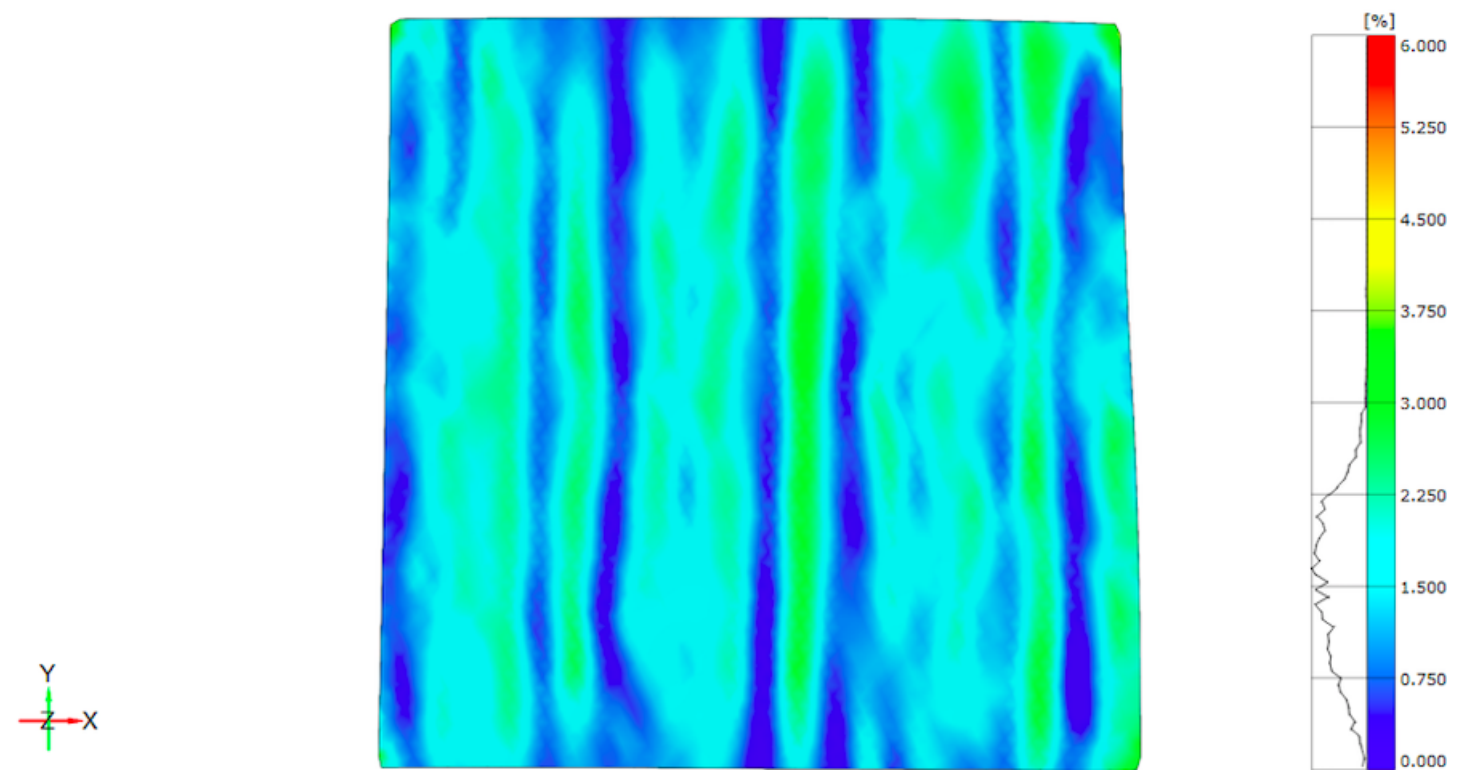

Figure 57: Biaxial tension state of 5 psi inflation pressure transverse strain field 


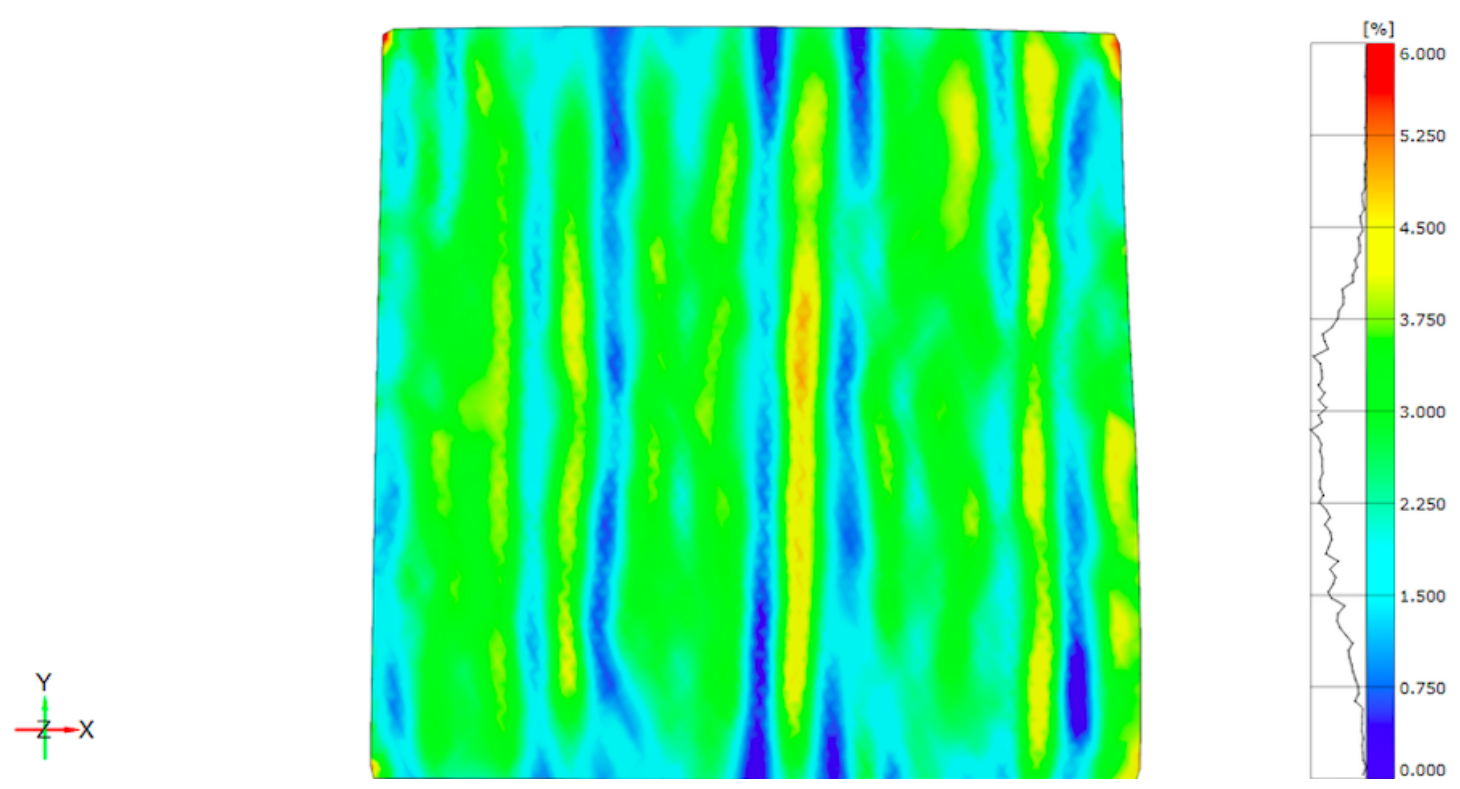

Figure 58: Biaxial tension state of 10 psi inflation pressure transverse strain field

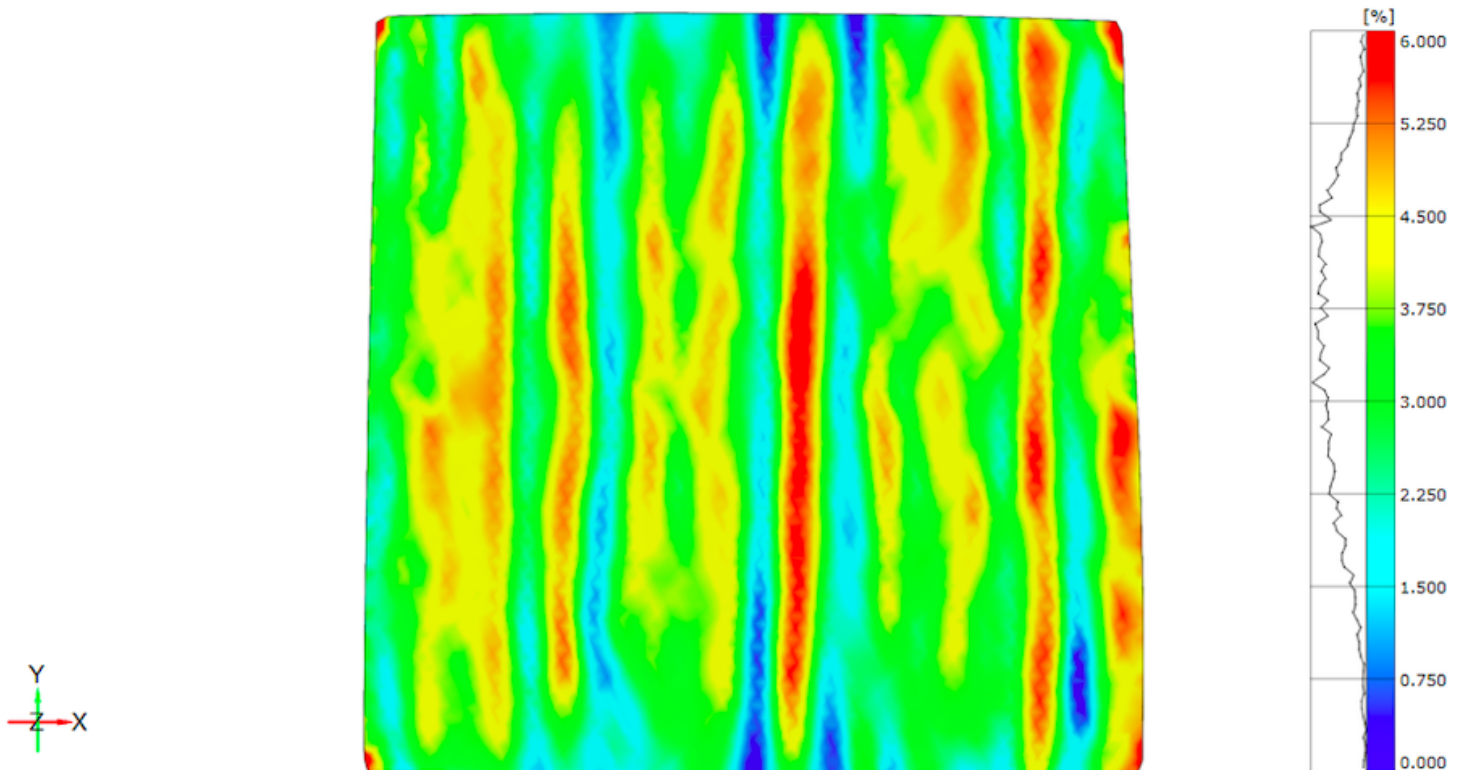

Figure 59: Biaxial tension state of 15 psi inflation pressure transverse strain field

Figure 60 is a stress strain curve of the warp and weft skin directions obtained by testing the orthogonal fibers together in a biaxial tensile test. Like the previous plot these results are compared to that of the uniaxial tensile testing done in this research with the same line convention. 


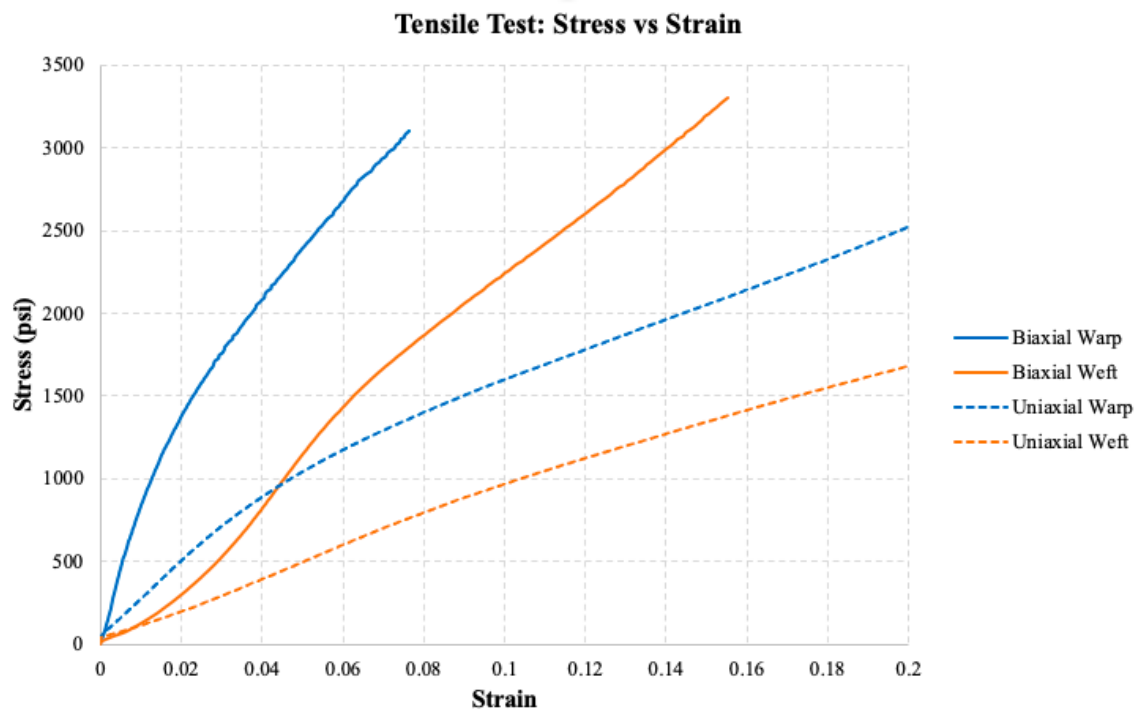

Figure 60: Biaxial and uniaxial tension stress vs strain

As shown by figures 53 and 60, the drop-stitch inflatable panel skin exhibits a different response to loading when both fiber directions are loaded as one rather than individually.

Equations 12 were then used with measured stresses and strains and the experimentally determined Poisson's ratios at each inflation state to calculate the the pressure dependent elastic modulus values given in table 6 .

Table 6: Experimentally determined elastic moduli from biaxial loading

\begin{tabular}{c|cc} 
Inflation Pressure (psi) & Warp: $\mathbf{E}_{1}($ psi) & Weft: $\mathbf{E}_{2}($ psi) \\
\hline $\mathbf{5}$ & 7,823 & 8,603 \\
$\mathbf{1 0}$ & 9,591 & 10,141 \\
$\mathbf{1 5}$ & 10,816 & 11,395
\end{tabular}

\subsection{Inflation testing}

Figures 61 through 63 show the axial surface strains of a local region of the drop-stitch panel due to inflation at 5, 10, and 15 psi inflations pressures. Notice the array of compressive strain regions are due to the internal drop yarns being 
tensioned by the panel inflating with air and pulling the panel skin, while the regions in between the drop yarns exhibit tensile strains because these regions are free to inflate.
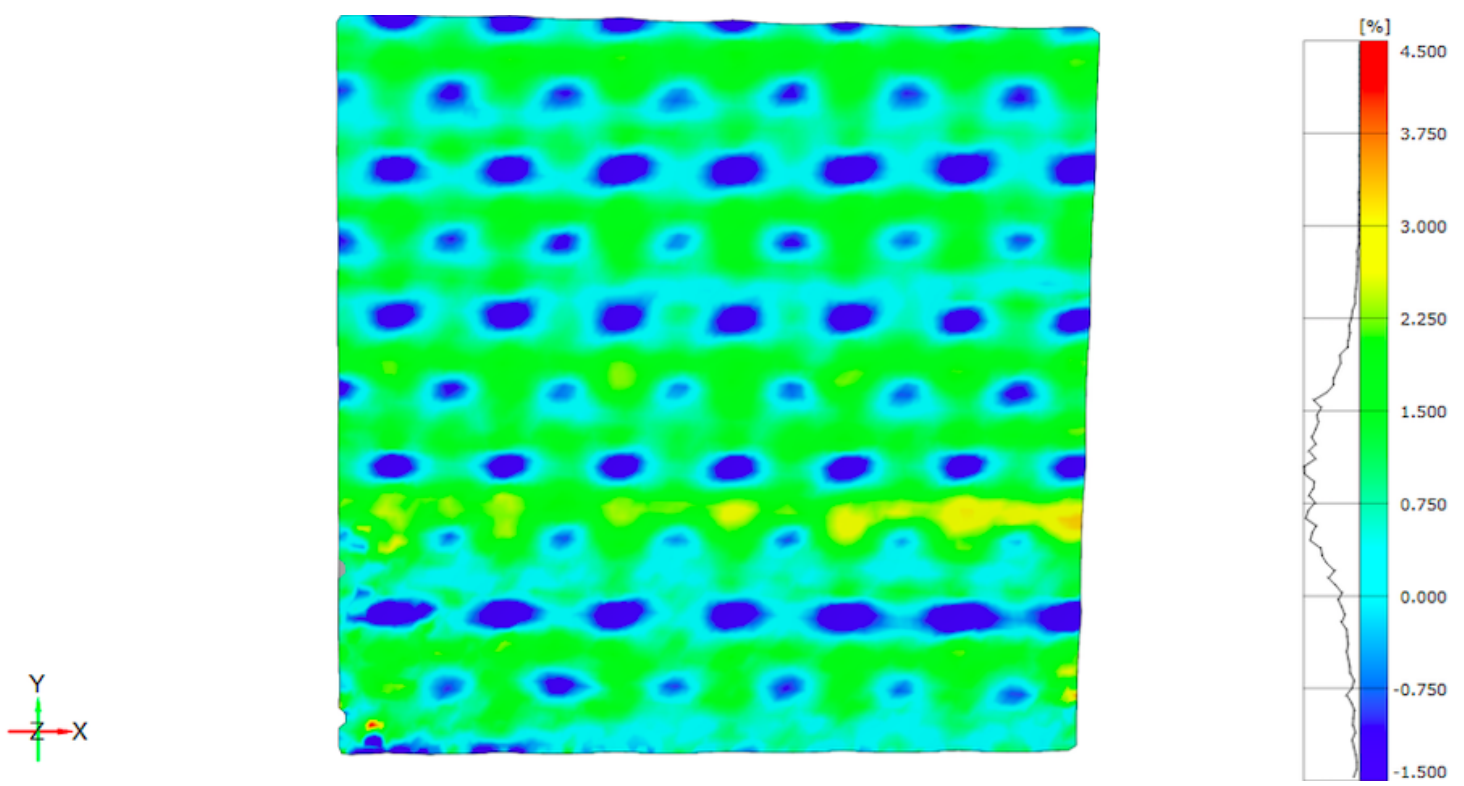

Figure 61: 5 psi inflation pressure axial strain
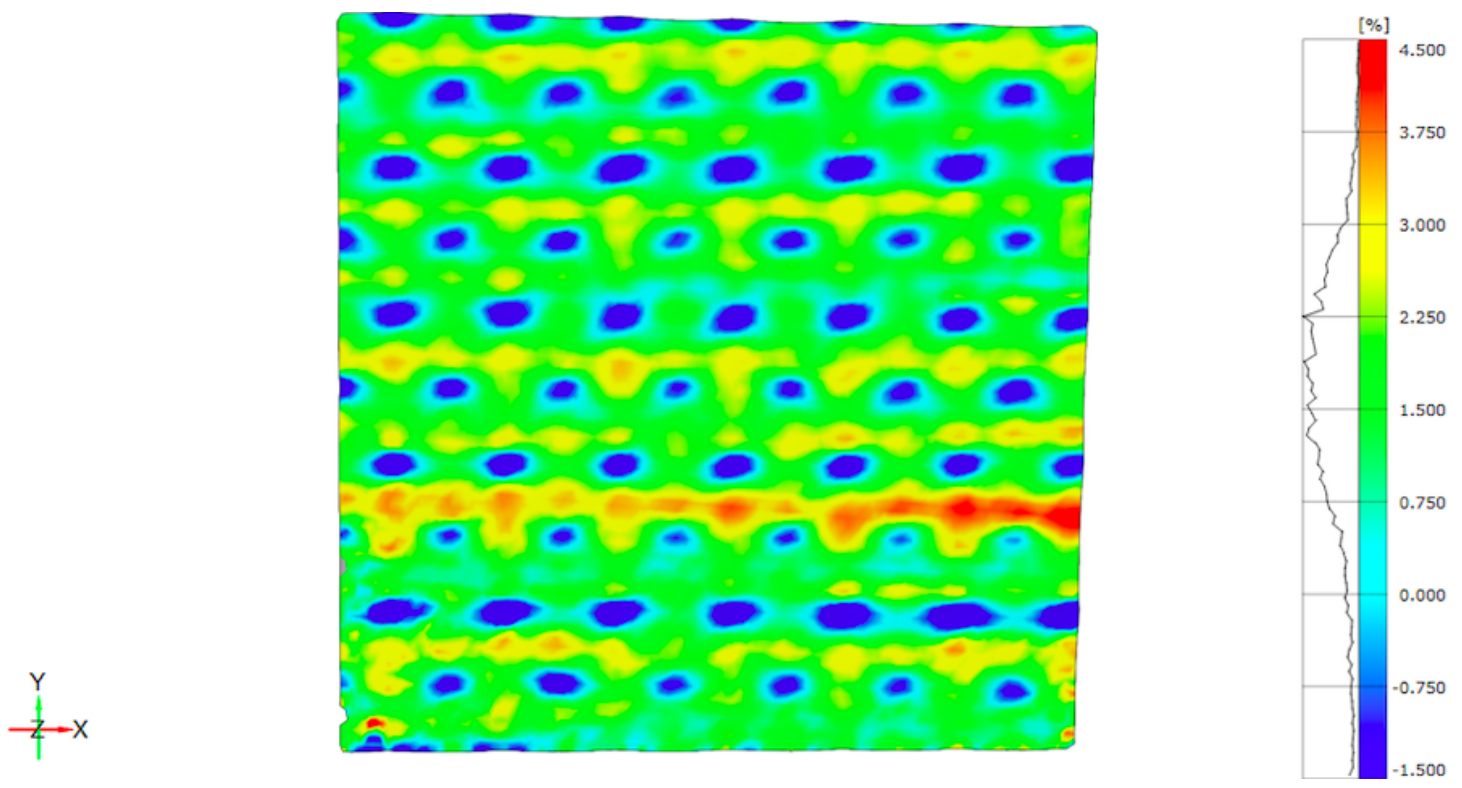

Figure 62: 10 psi inflation pressure axial strain 


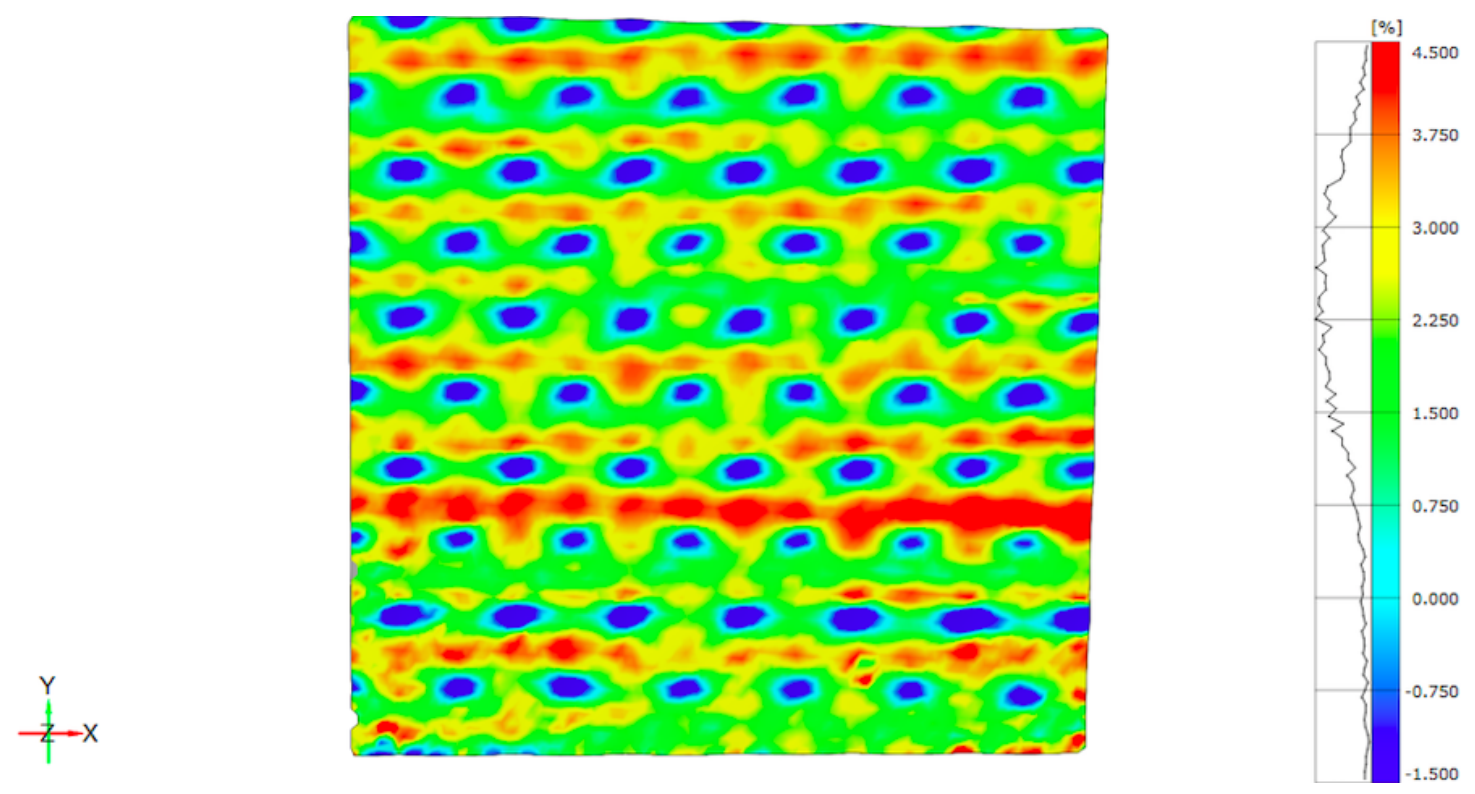

Figure 63: 15 psi inflation pressure axial strain

Figure 64 shows the axial strain along a section of the analyzed region. This section is a straight line measured from the top of the analyzed region. The positive tensile strain regions of the profile show the axial surface strains in between the drop yarns while the negative compressive axial strain regions of the profile show the location of said drop yarns. As shown by the figure, the drop yarns are spaced about 0.5 inches apart internally. 


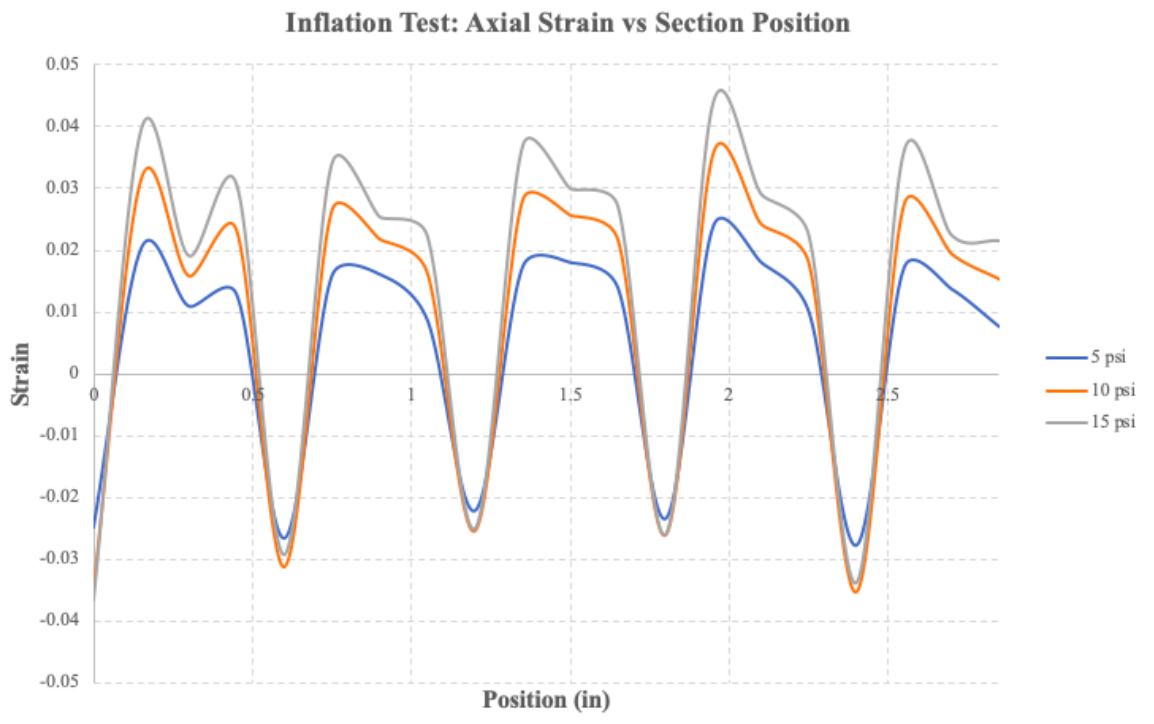

Figure 64: Axial strain profile of local section

Figures 65 through 67 show the transverse surface strains of the same local region of the drop-stitch panel due to inflation at 5, 10, and 15 psi inflation pressures. Notice again the array of compressive strain regions are due to the internal drop yarns being tensioned by the panel inflating with air and pulling the panel skin, while the regions in between the drop yarns again exhibit tensile strains because these regions are free to inflate. 

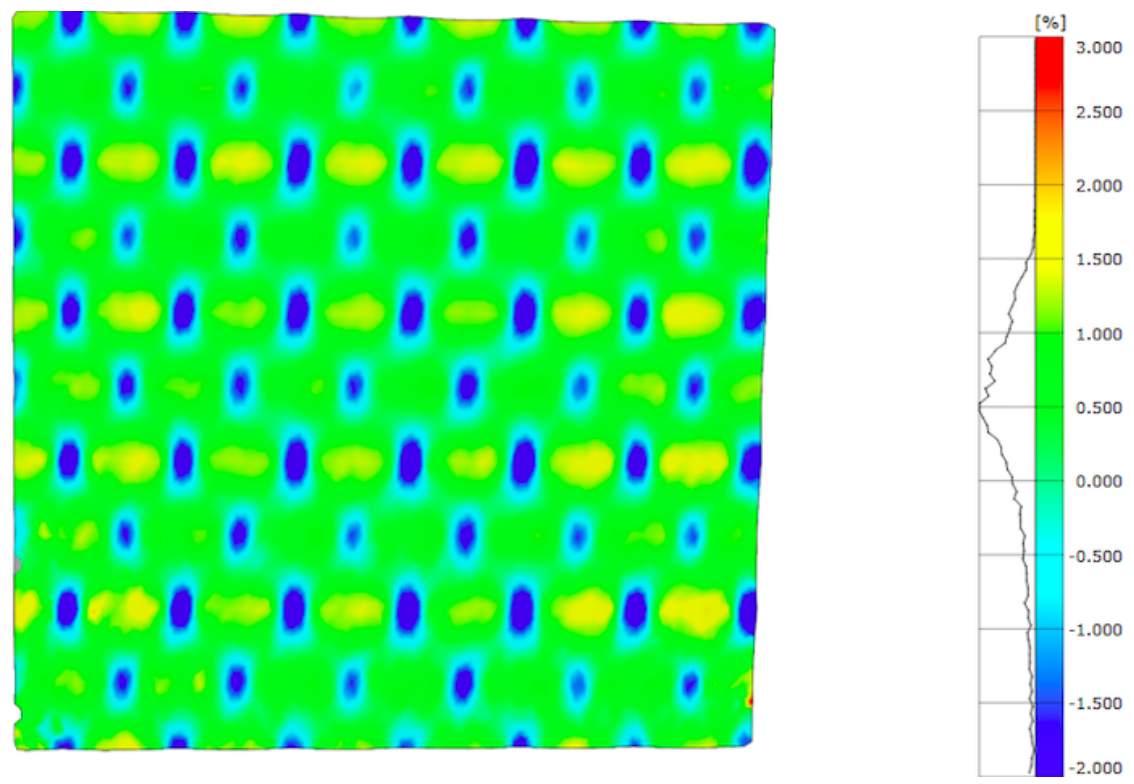

Figure 65: 5 psi inflation pressure transverse strain
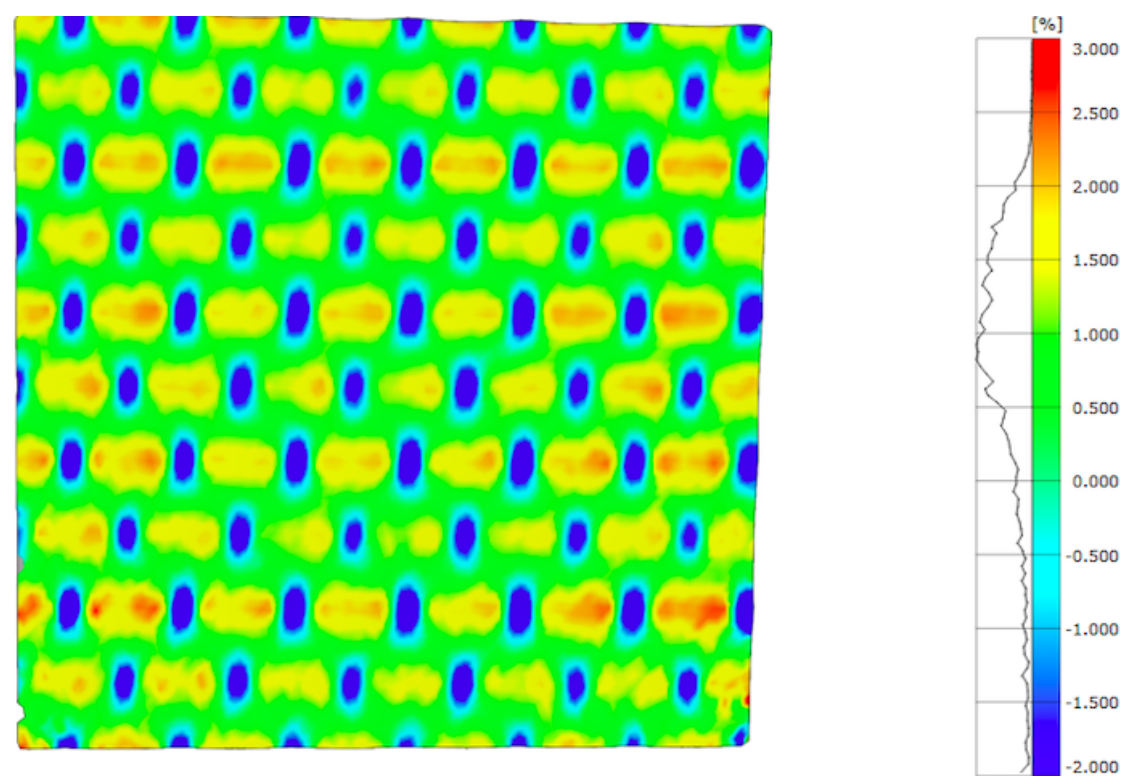

Figure 66: 10 psi inflation pressure transverse strain 


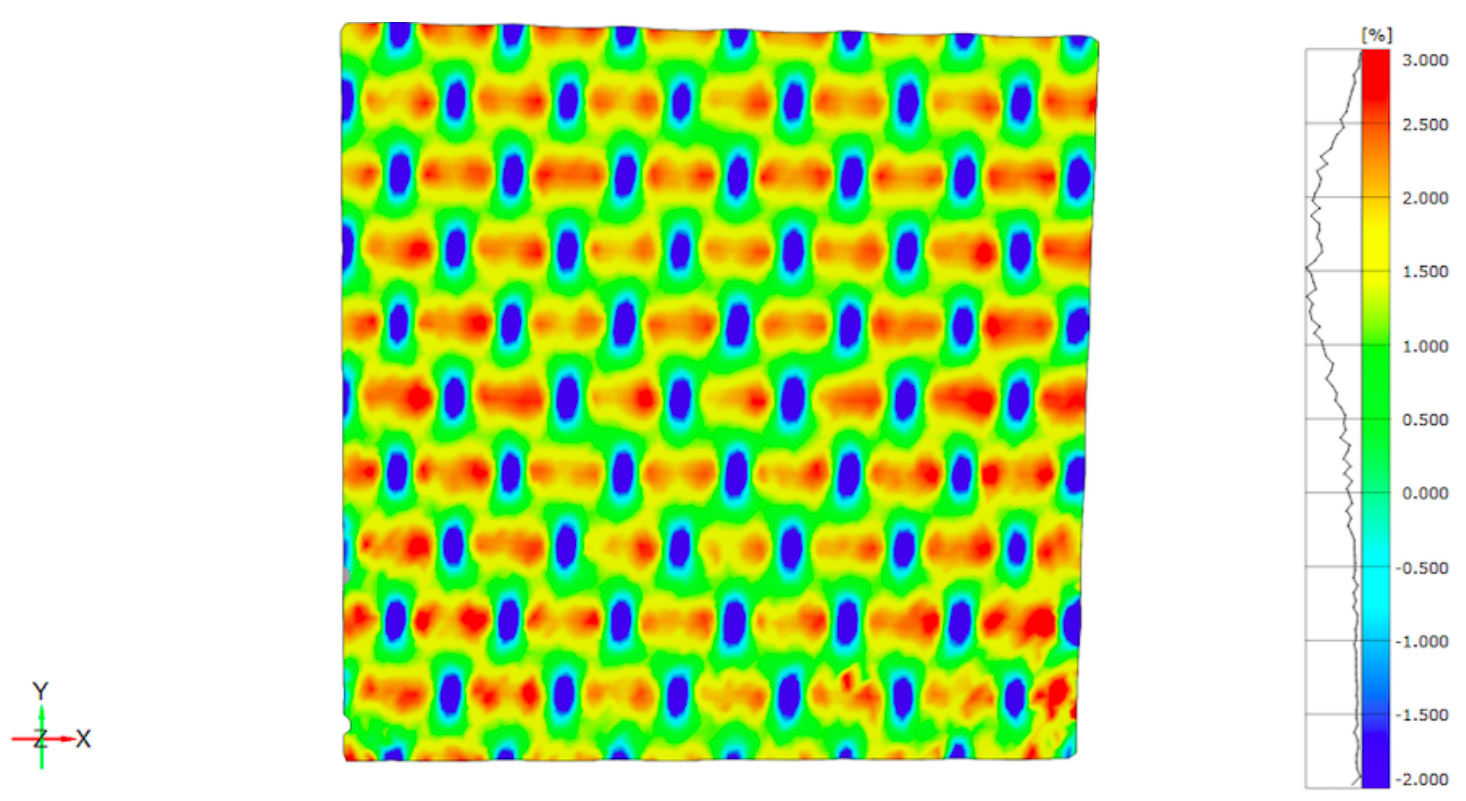

Figure 67: 15 psi inflation pressure transverse strain

Figure 68 shows the transverse strain along a section of the analyzed region. The same section was analyzed as in figure 64 . The positive tensile strain regions of the profile again show the transverse surface strains in between the drop yarns while the negative compressive transverse strain regions of the profile show the location of said drop yarns.

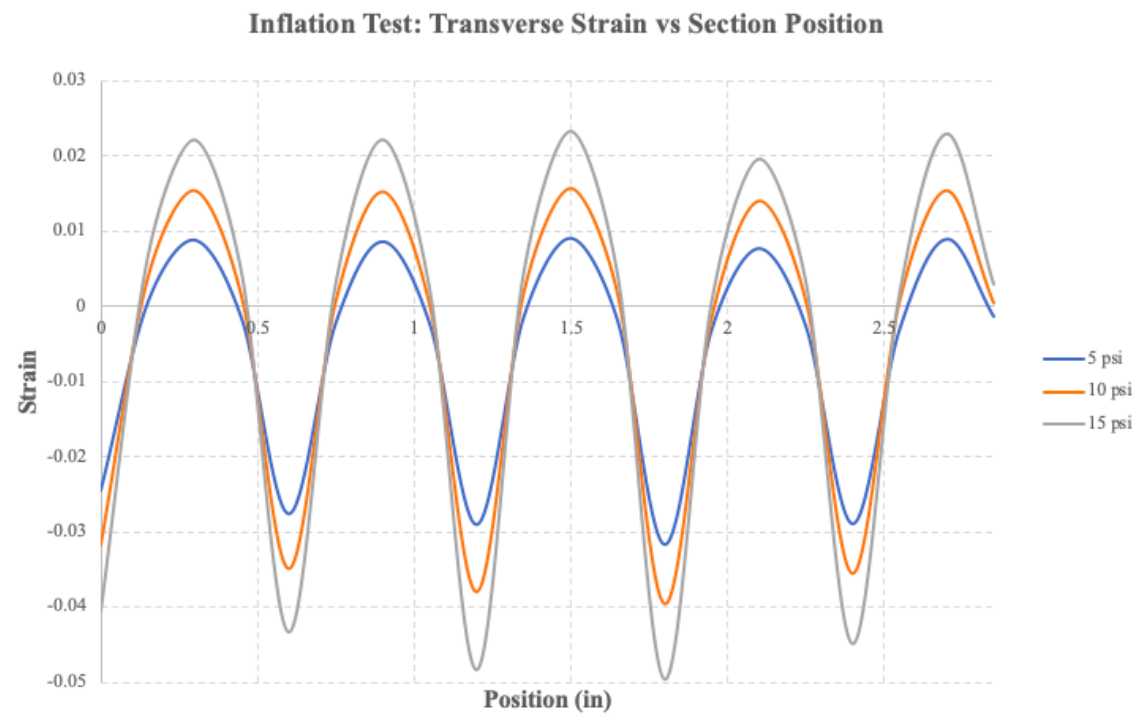

Figure 68: Transverse strain profile of local section 
Figure 69 shows a plot of drop-stitch panel skin stresses and strains due to inflation. The axial and transverse inflation stresses from 0 psi to 15 psi were calculated in a MATLAB program using equation 23. These stress values were then plotted against average axial and transverse strains due to inflation measured by digital image correlation.

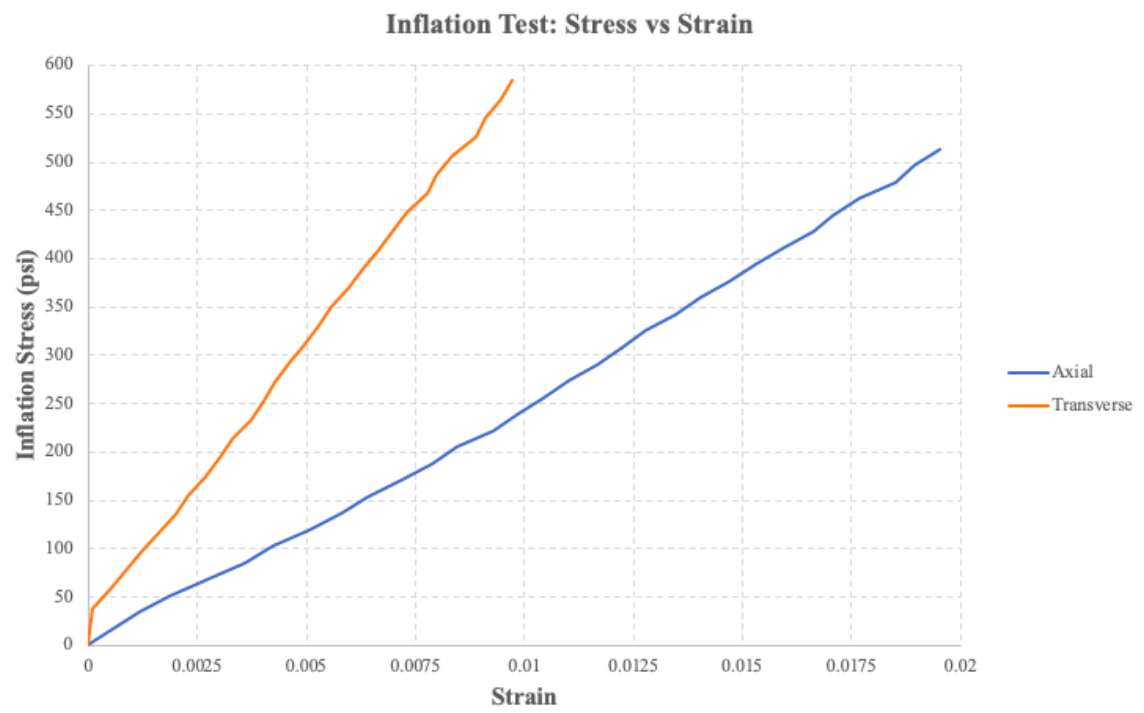

Figure 69: Axial and transverse inflation pressures vs strains

Using equation 12, calculated axial and transverse inflation stresses, measured axial and transverse surface strains from digital image correlation and experimentally determined Poisson's ratios, elastic modulus values from inflation can be calculated as shown in table 7 .

Table 7: Experimentally determined elastic moduli from inflation

\begin{tabular}{c|cc} 
Inflation Pressure (psi) & Warp: $\mathbf{E}_{1}($ psi) & Weft: $\mathbf{E}_{2}($ psi) \\
\hline $\mathbf{5}$ & 10,863 & 21,572 \\
$\mathbf{1 0}$ & 11,207 & 21,752 \\
$\mathbf{1 5}$ & 11,349 & 21,743
\end{tabular}




\subsection{Four-point bend testing}

Figure 70 shows the measured load vs deflection curves of the drop-stitch inflatable panel in four-point bending at 5,10 and 15 psi. These tests were used to analyze the top surface of the panel.

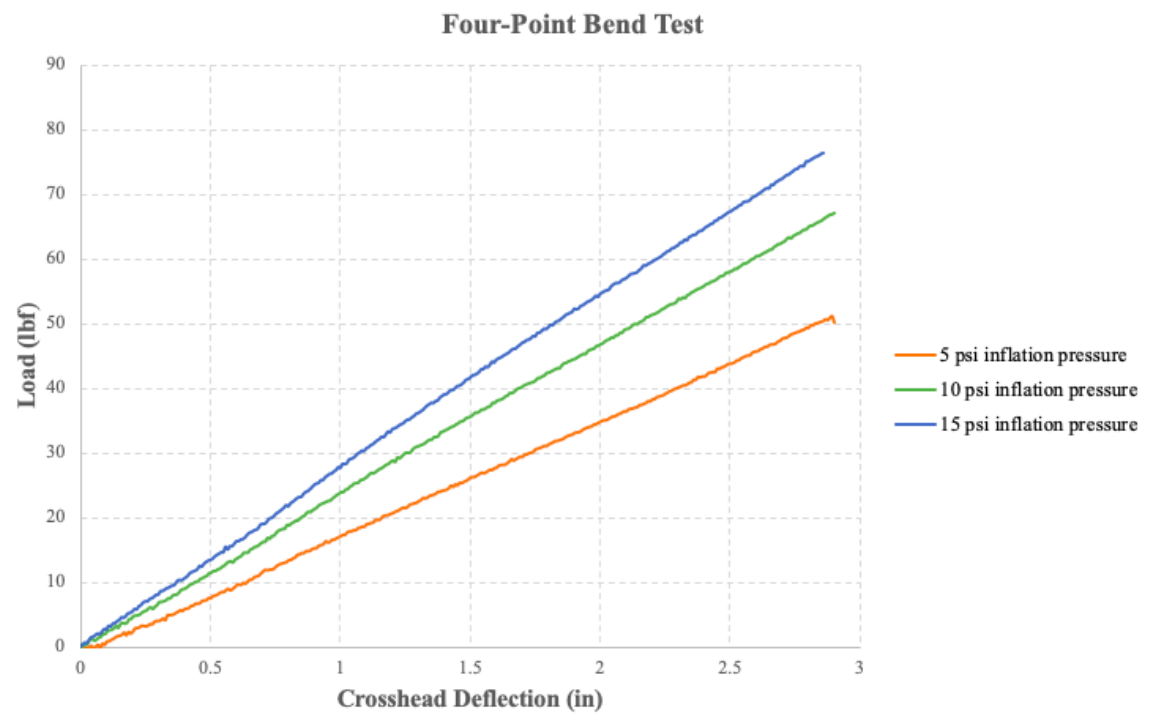

Figure 70: Four-point bending panel top surface testing load vs deflection

Table 9 gives the stiffness values of the above four-point bending tests and the measured load at the maximum programmed 3 inch deflection.

Table 8: Four-point bend stiffness and load at maximum deflection of bend tests analyzing the panel top surface

\begin{tabular}{c|cc} 
Inflation Pressure (psi) & Stiffness (lb/in) & Load at Deflection (lbf) \\
\hline $\mathbf{5}$ & 17.92 & 50.11 \\
$\mathbf{1 0}$ & 23.25 & 67.19 \\
$\mathbf{1 5}$ & 26.81 & 76.26
\end{tabular}

Figures 71 through 73 show the panel top surface deflection at the point that the load frame cross head reached it max deflection at 5,10 and 15 psi inflation pressures. Notice that although it will be shown later that panel bottom surface does deflect the full 3 inches, the top surface deflection is slightly lower. This is 
due to the compressibility of air inside the panel causing wrinkling at the panel top surface.

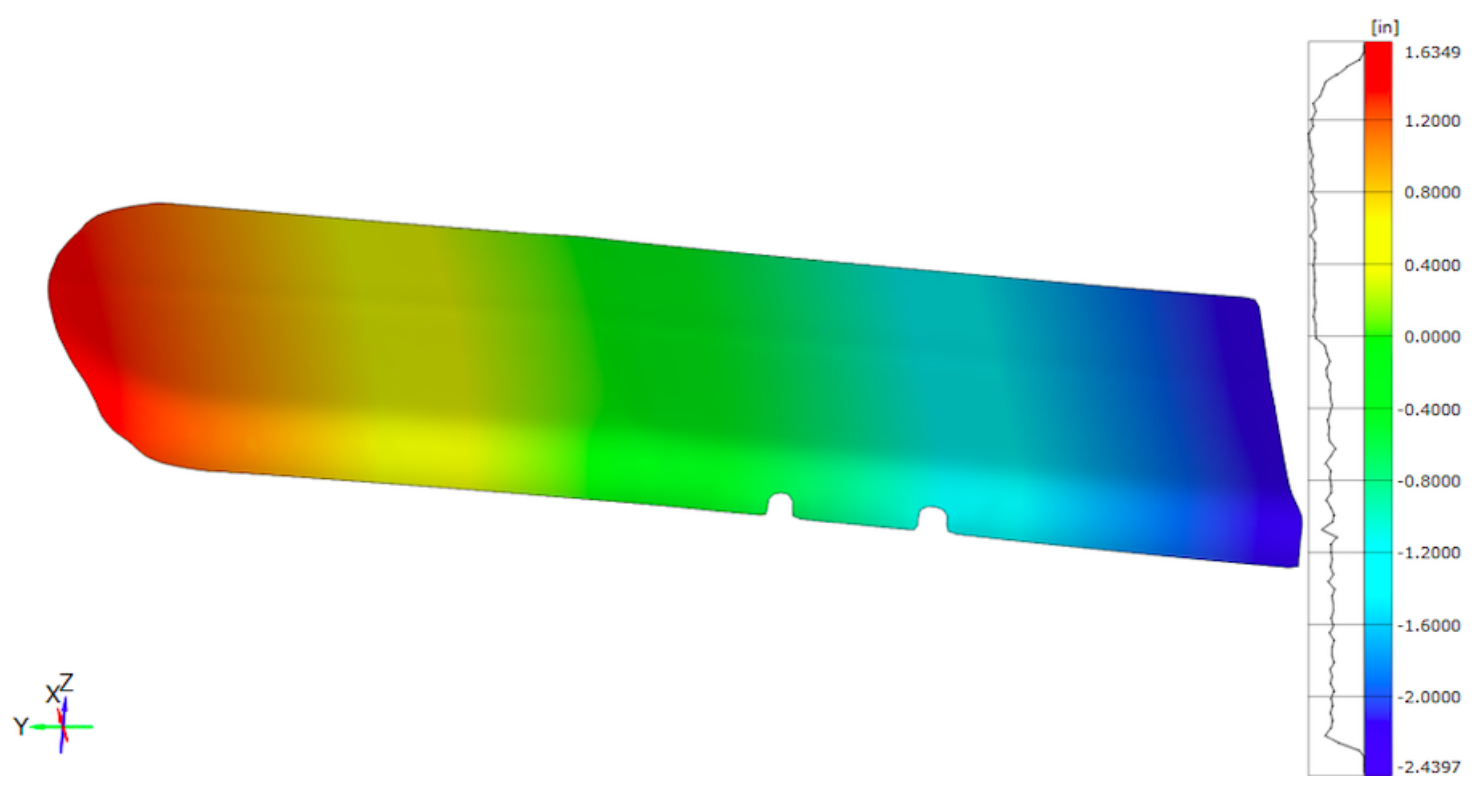

Figure 71: Four-point bending panel top surface deflection at 5 psi inflation pressure

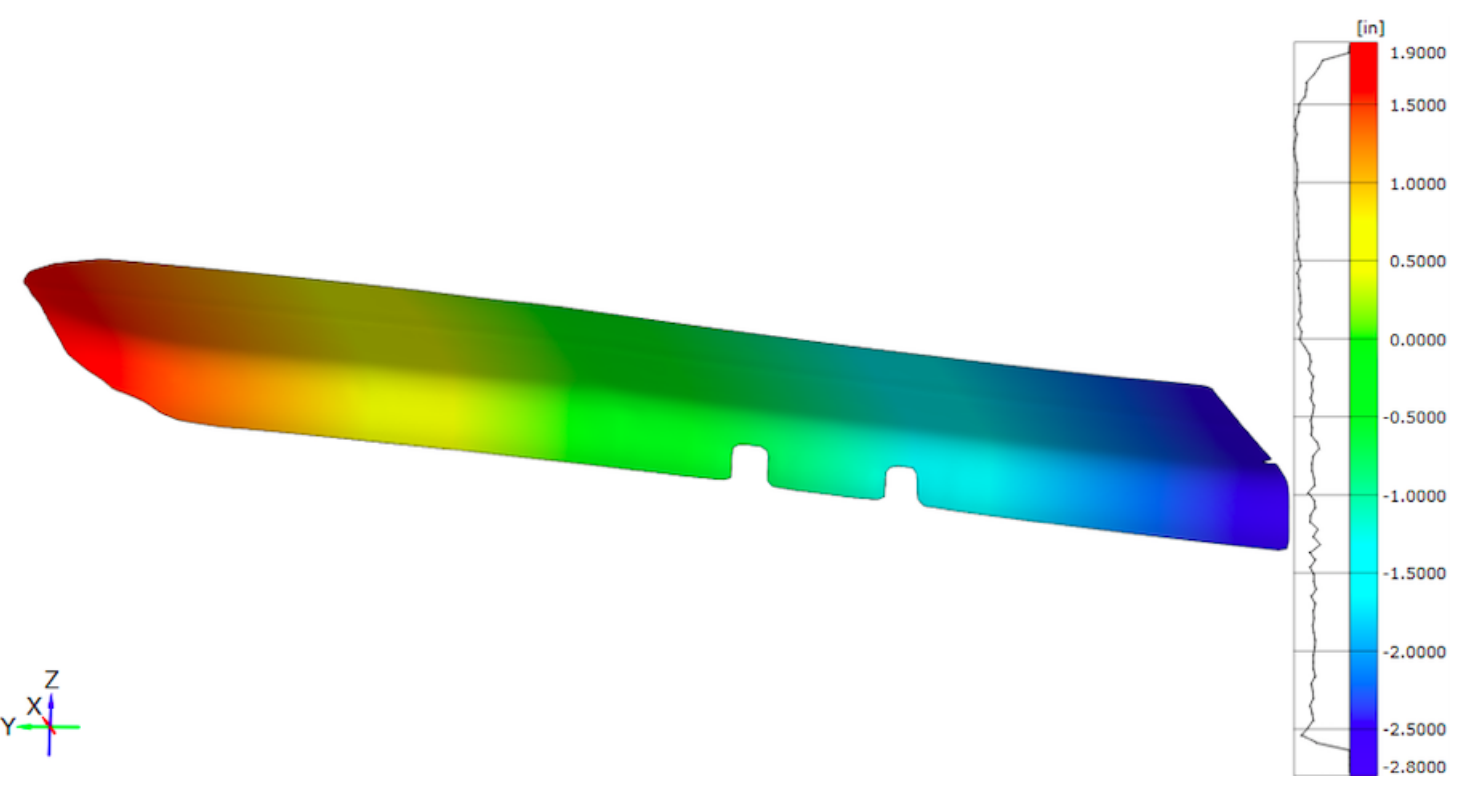

Figure 72: Four-point bending panel top surface deflection at 10 psi inflation pressure 


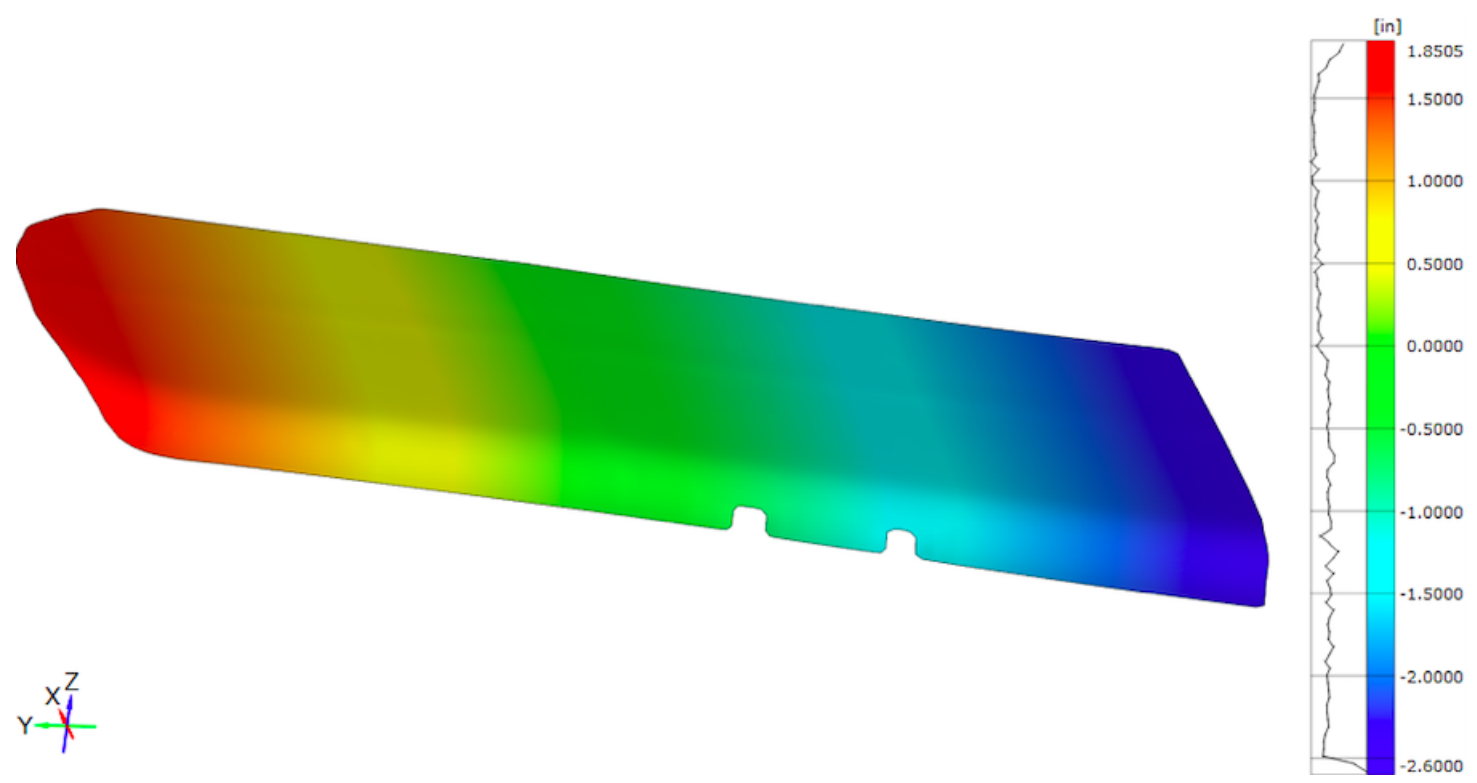

Figure 73: Four-point bending panel top surface deflection at 15 psi inflation pressure

Figures 74 through 76 show the panel top surface axial strain field at the point that the load frame cross head reached it max deflection at 5,10 and 15 psi inflation pressures. Notice that as expected in beam bending, the panel top surface has a large region of compressive strain focused near the loading pin. 


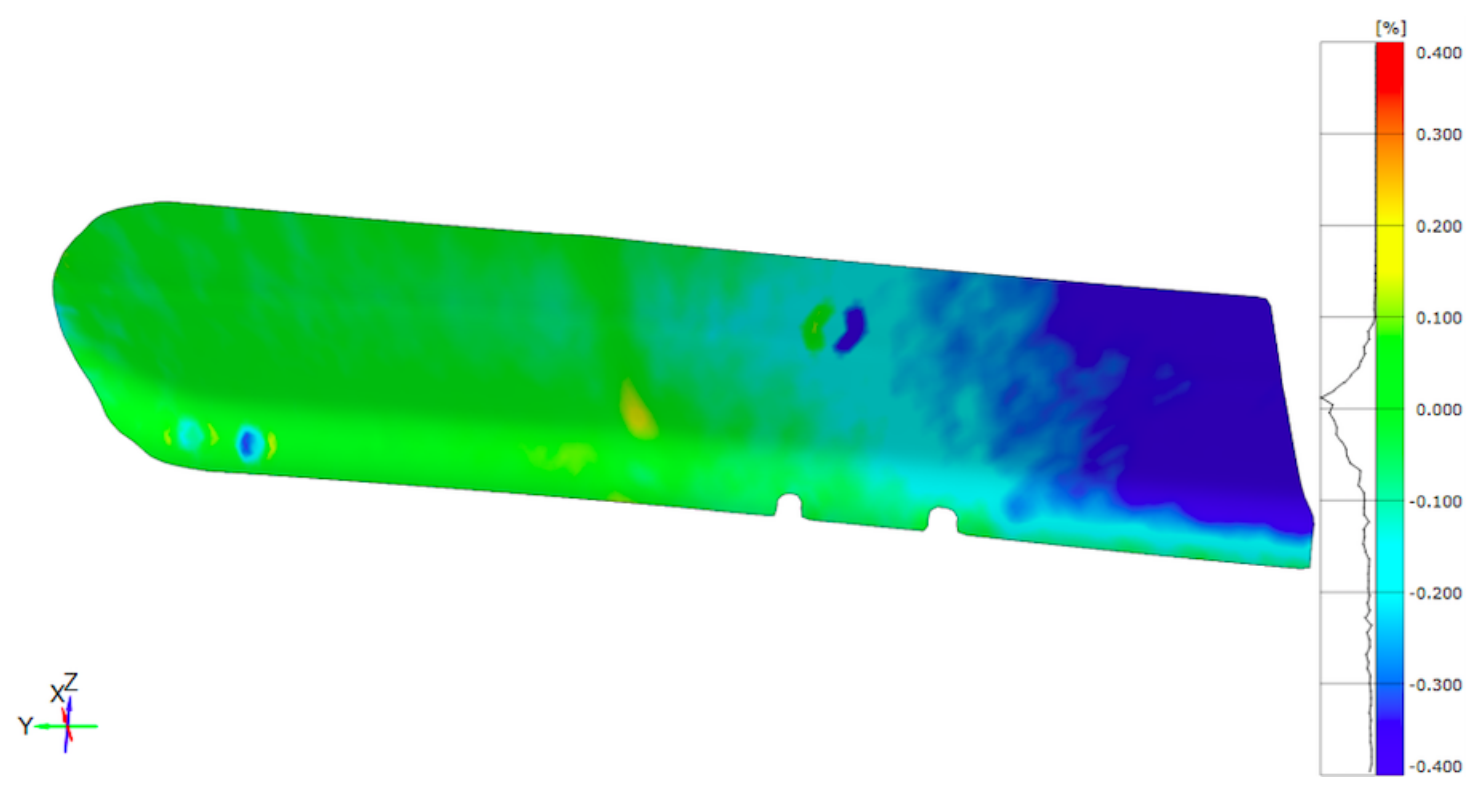

Figure 74: Four-point bending panel top surface axial strain at 5 psi inflation pressure

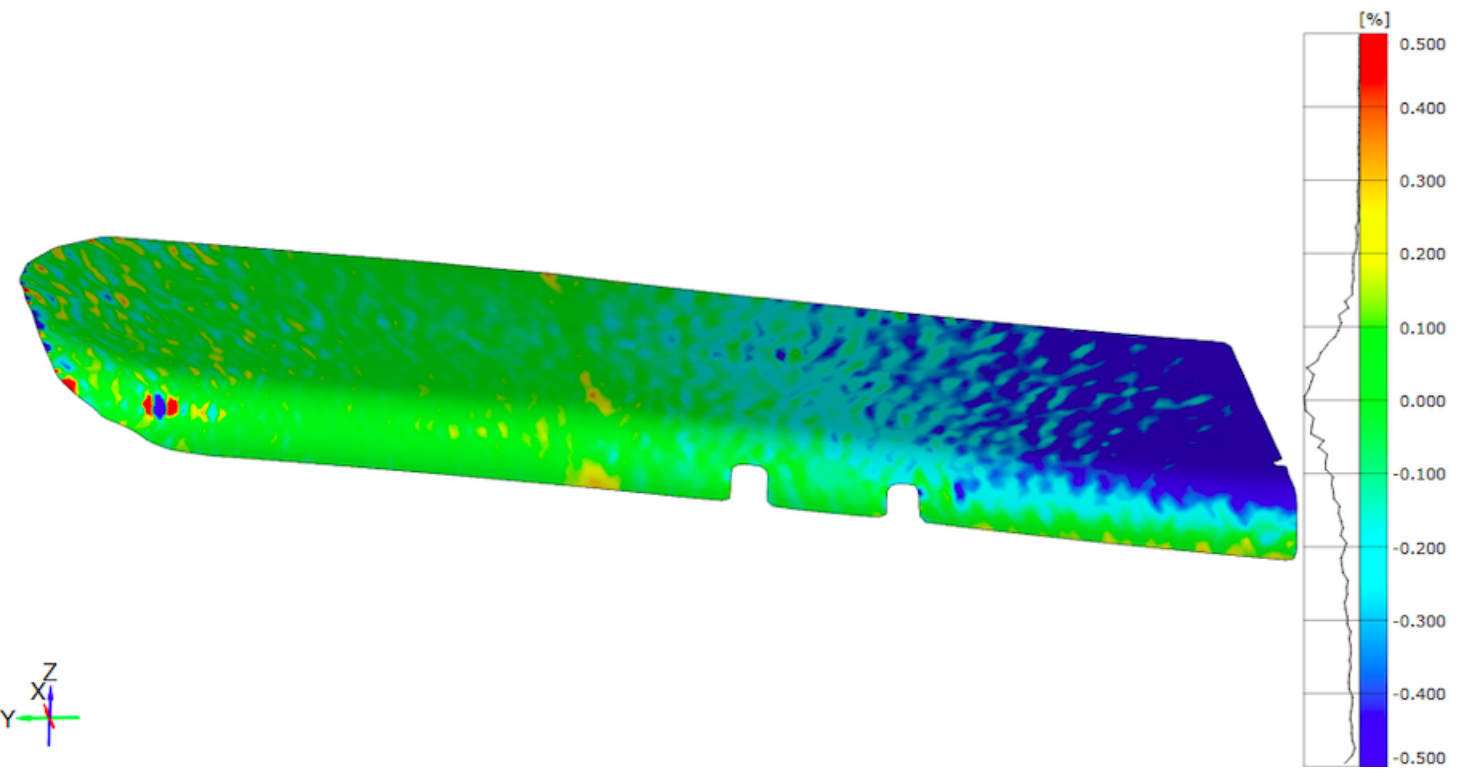

Figure 75: Four-point bending panel top surface axial strain at 10 psi inflation pressure 


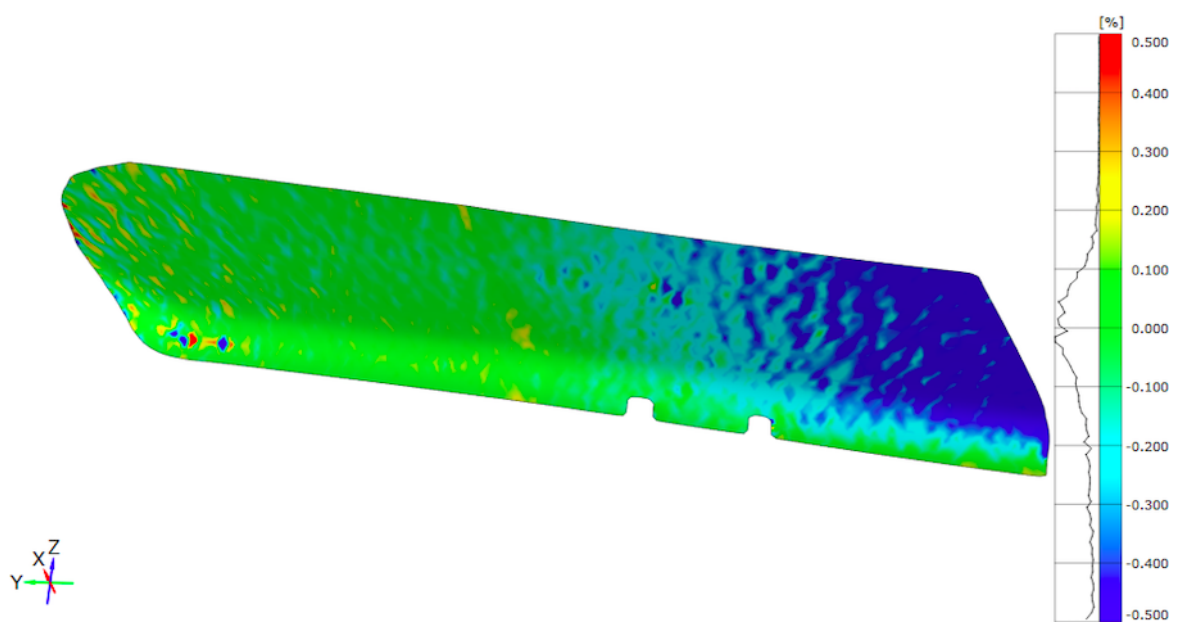

Figure 76: Four-point bending panel top surface axial strain at 15 psi inflation pressure

Figures 77 through 79 show the panel top surface transverse strain field at the point that the load frame cross head reached it max deflection at 5,10 and 15 psi inflation pressures. The transverse strain fields produced were extremely nonuniform, and observed at every inflation pressure.

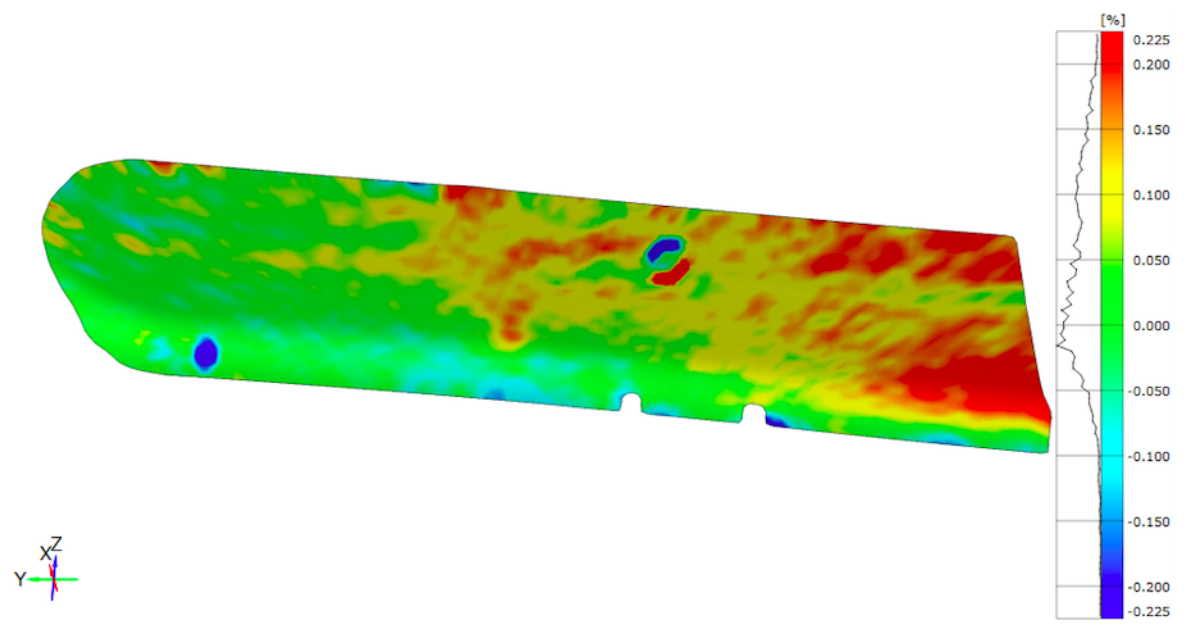

Figure 77: Four-point bending panel top surface transverse strain at 5 psi inflation pressure 


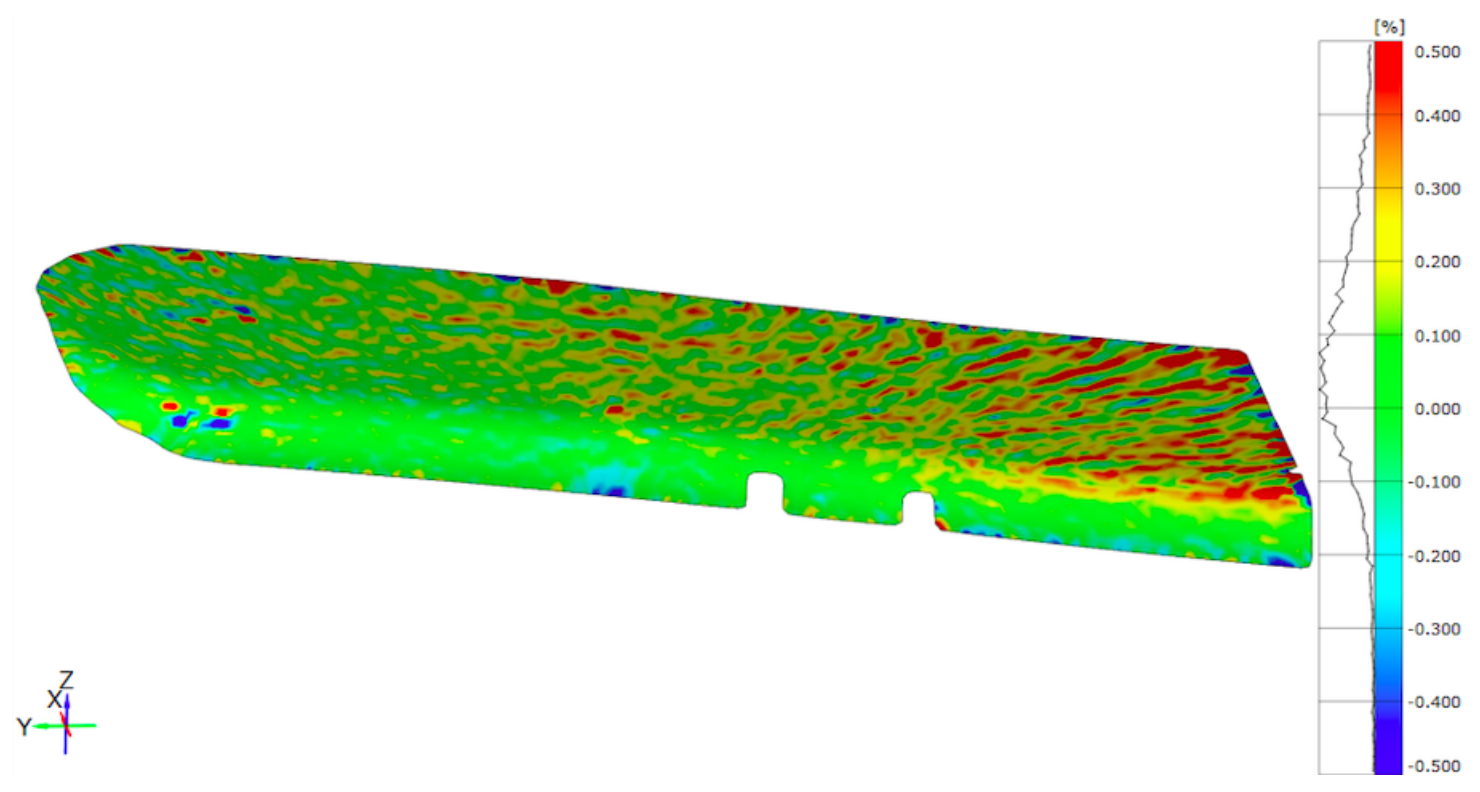

Figure 78: Four-point bending panel top surface transverse strain at 10 psi inflation pressure

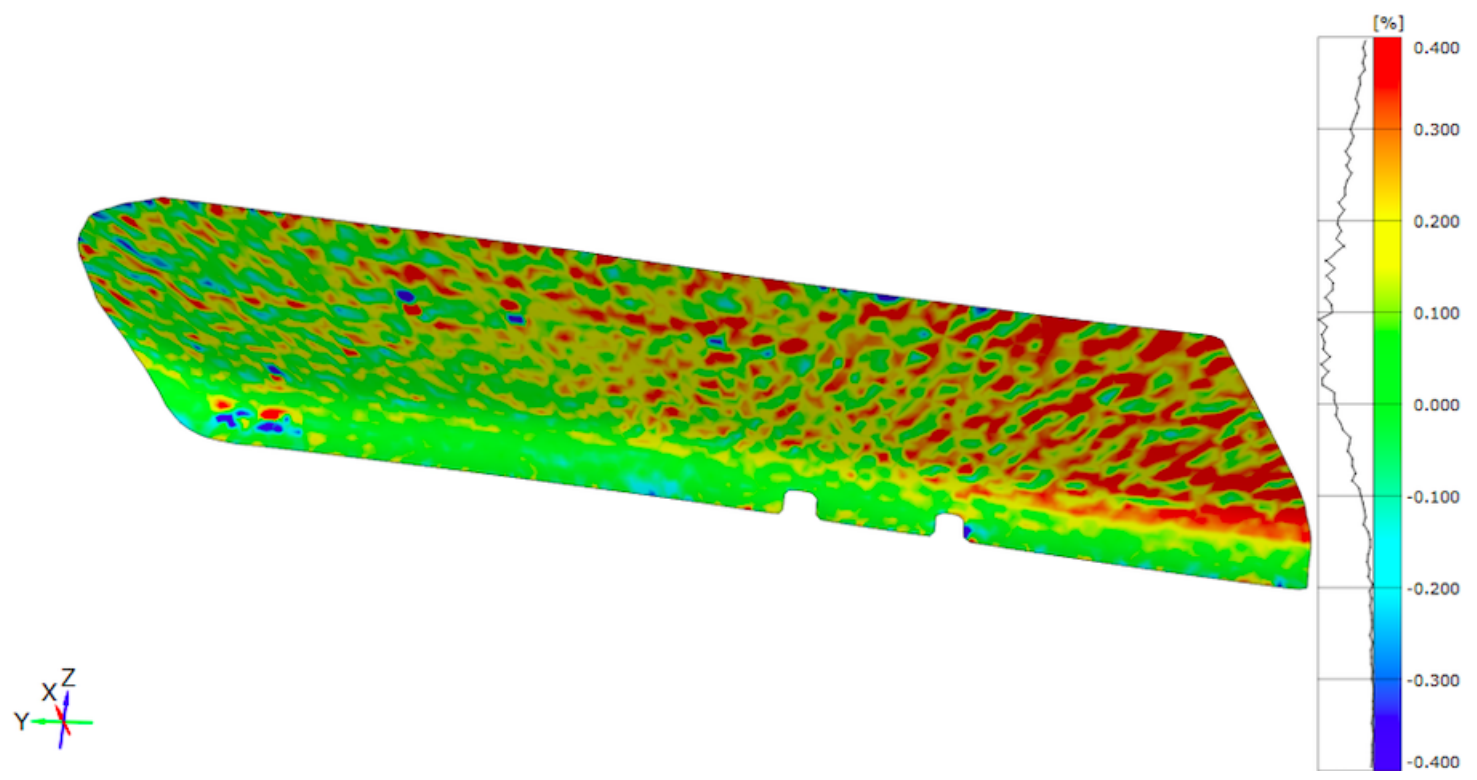

Figure 79: Four-point bending panel top surface transverse strain at 15 psi inflation pressure

Figure 80 shows the measured load vs deflection curves of the drop-stitch inflatable panel in four-point bending at 5,10 and 15 psi. These tests were used to analyze the bottom surface of the panel. 


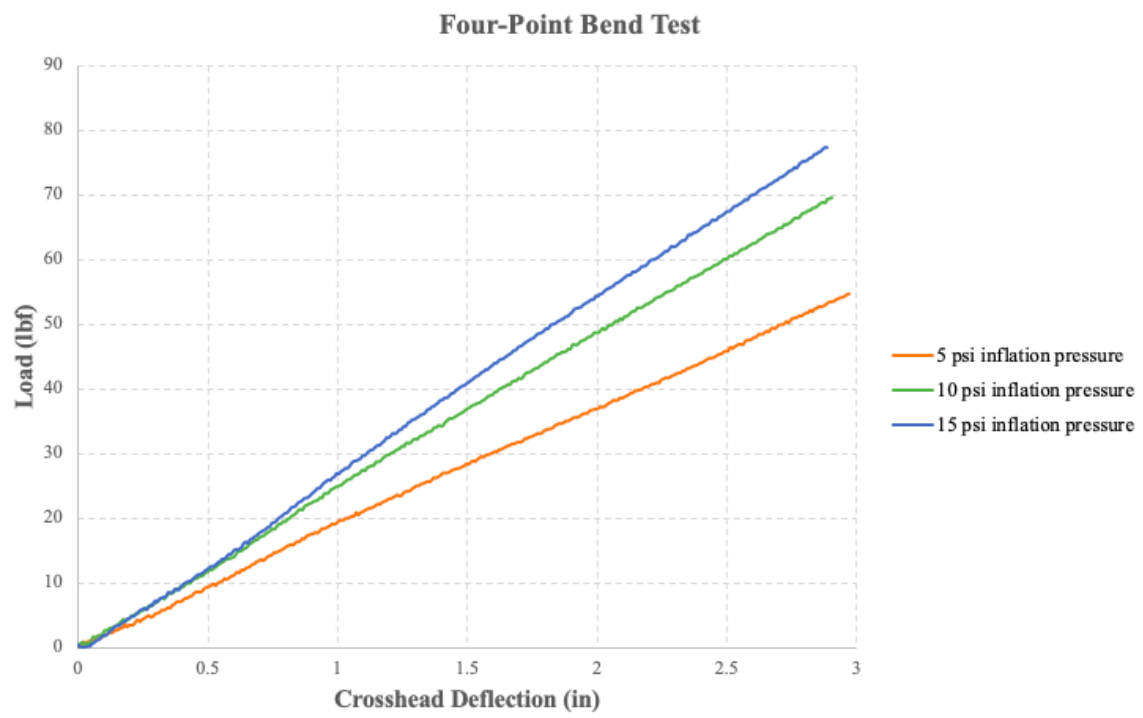

Figure 80: Four-point bending panel bottom surface testing load vs deflection

Table 9 gives the stiffness values of the above four-point bending tests and the measured load at the maximum programmed 3 inch deflection.

Table 9: Four-point bend stiffness and load at maximum deflection of bend tests analyzing the panel bottom surface

\begin{tabular}{c|cc} 
Inflation Pressure (psi) & Stiffness (lb/in) & Load at Deflection (lbf) \\
\hline $\mathbf{5}$ & 18.23 & 54.55 \\
$\mathbf{1 0}$ & 24.03 & 69.54 \\
$\mathbf{1 5}$ & 27.39 & 77.32
\end{tabular}

Figures 81 through 83 show the panel bottom surface deflection at the point that the load frame cross head reached it max deflection at 5,10 and 15 psi inflation pressures. The region of the bottom surface of the panel under the load pin did in fact deflect the full 3 inches as shown. 


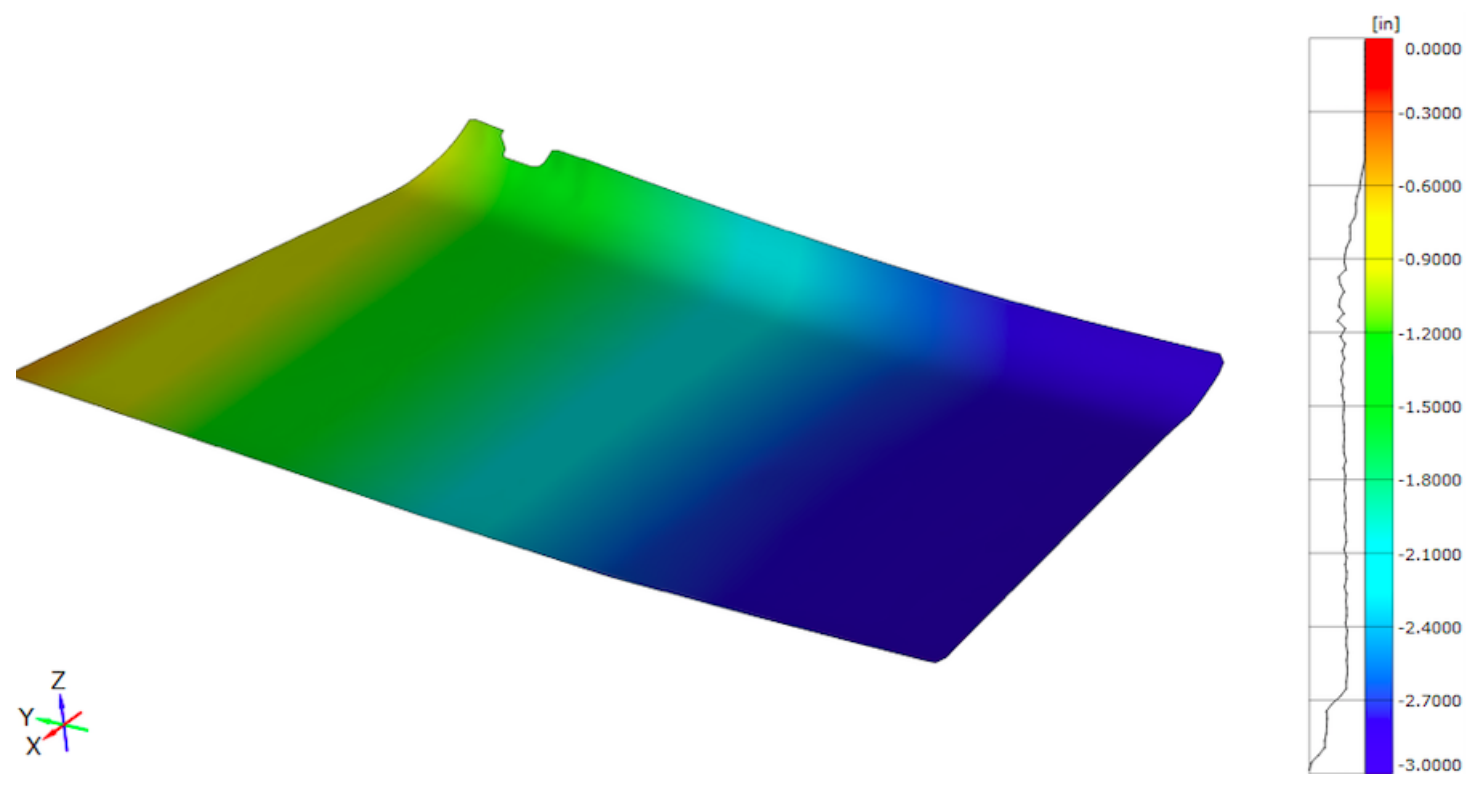

Figure 81: Four-point bending panel bottom surface deflection at 5 psi inflation pressure

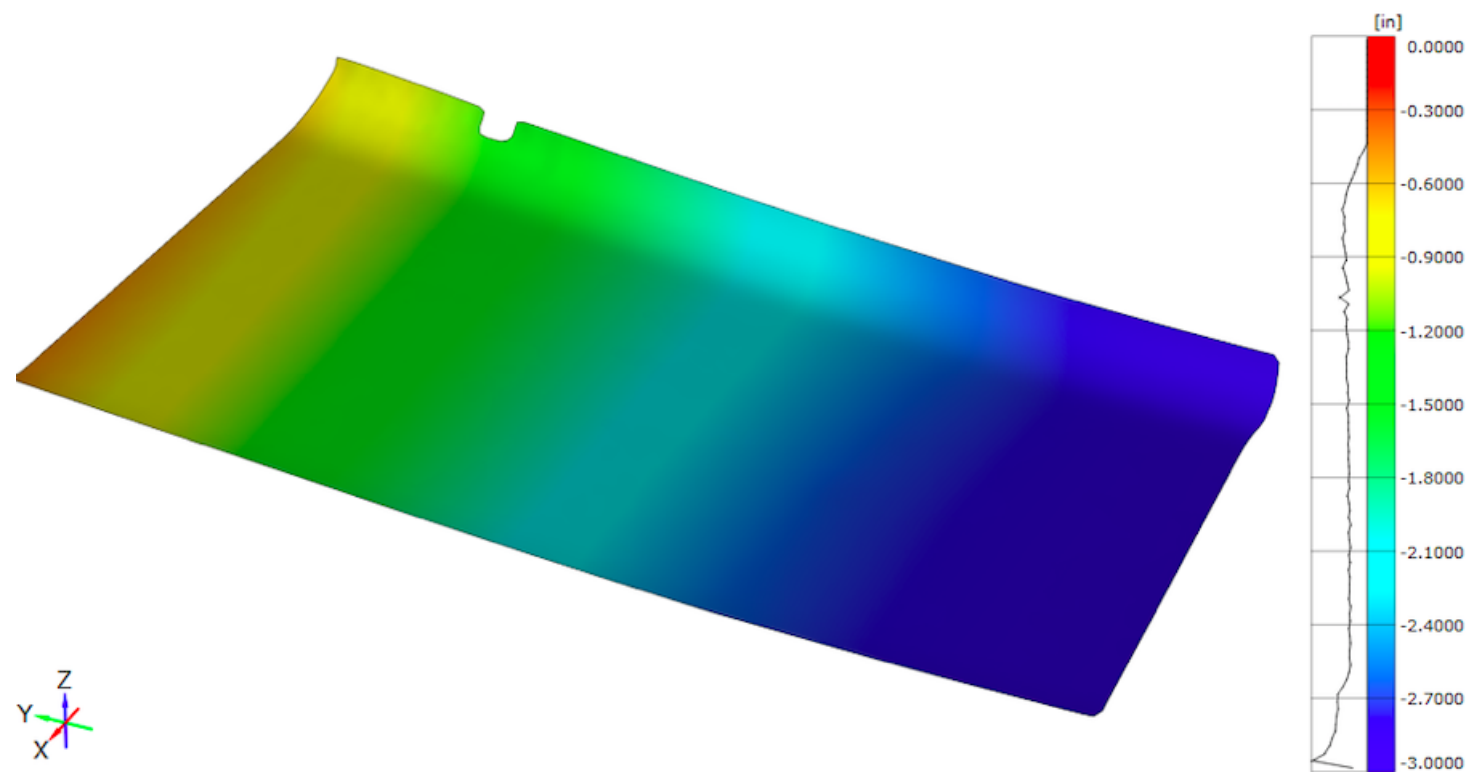

Figure 82: Four-point bending panel bottom surface deflection at 10 psi inflation pressure 


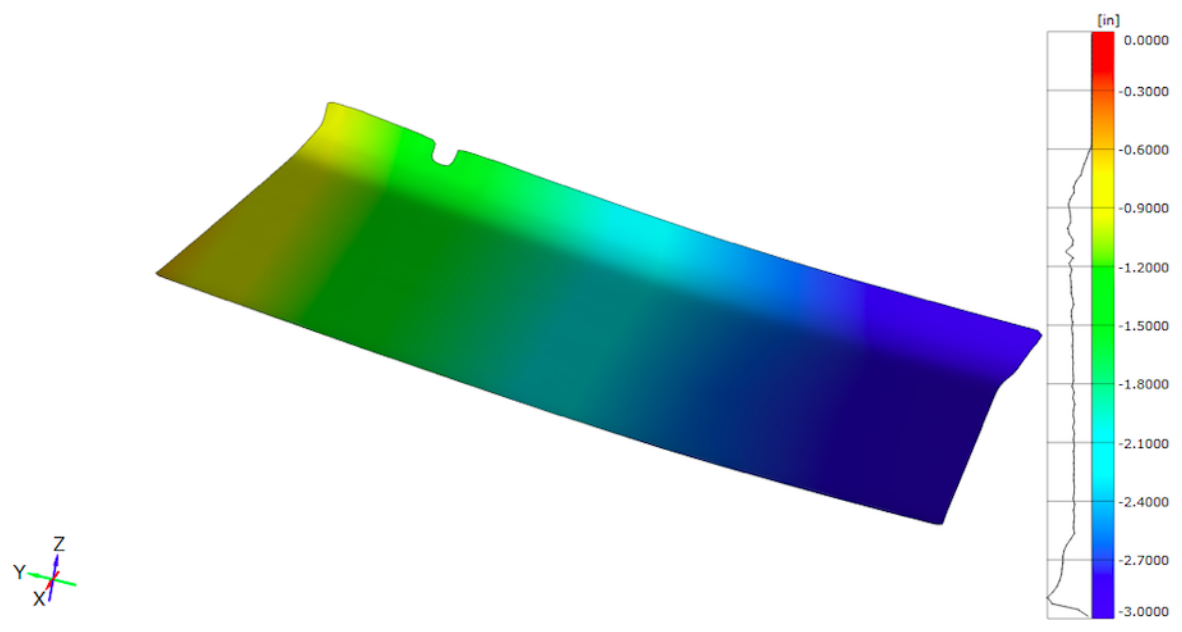

Figure 83: Four-point bending panel bottom surface deflection at 15 psi inflation pressure

Figures 74 through 76 show the panel bottom surface axial strain field at the point that the load frame cross head reached it max deflection at 5,10 and 15 psi inflation pressures. Notice that as expected in beam bending, the panel bottom surface has a large region of tensile strain focused under the loading pin.

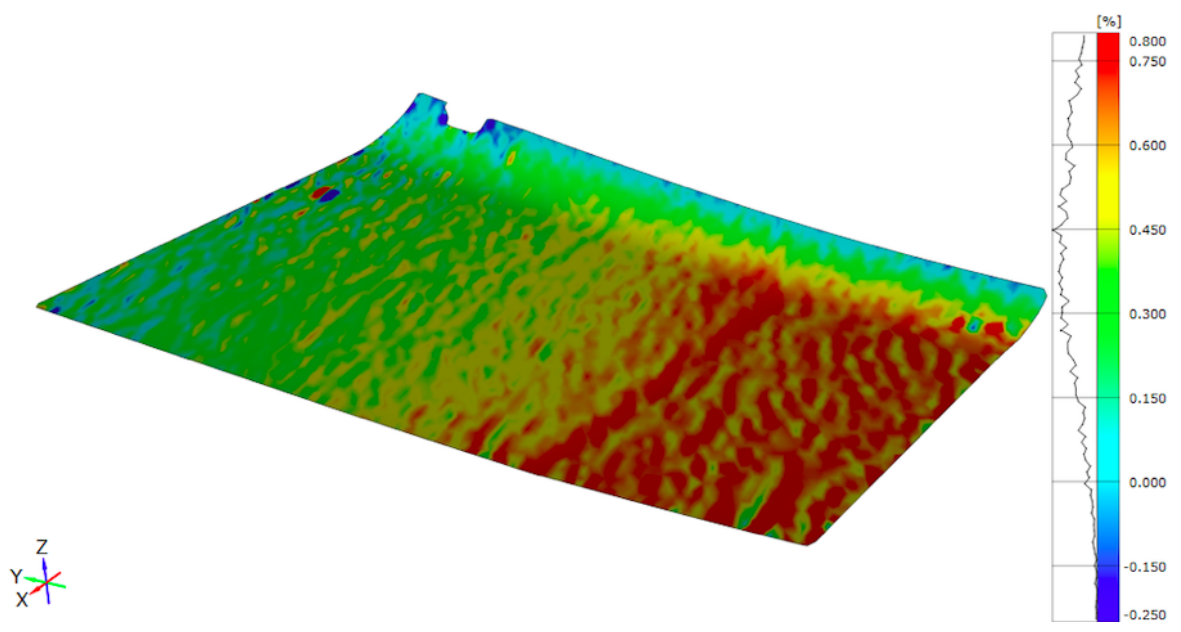

Figure 84: Four-point bending panel bottom surface axial strain at 5 psi inflation pressure 

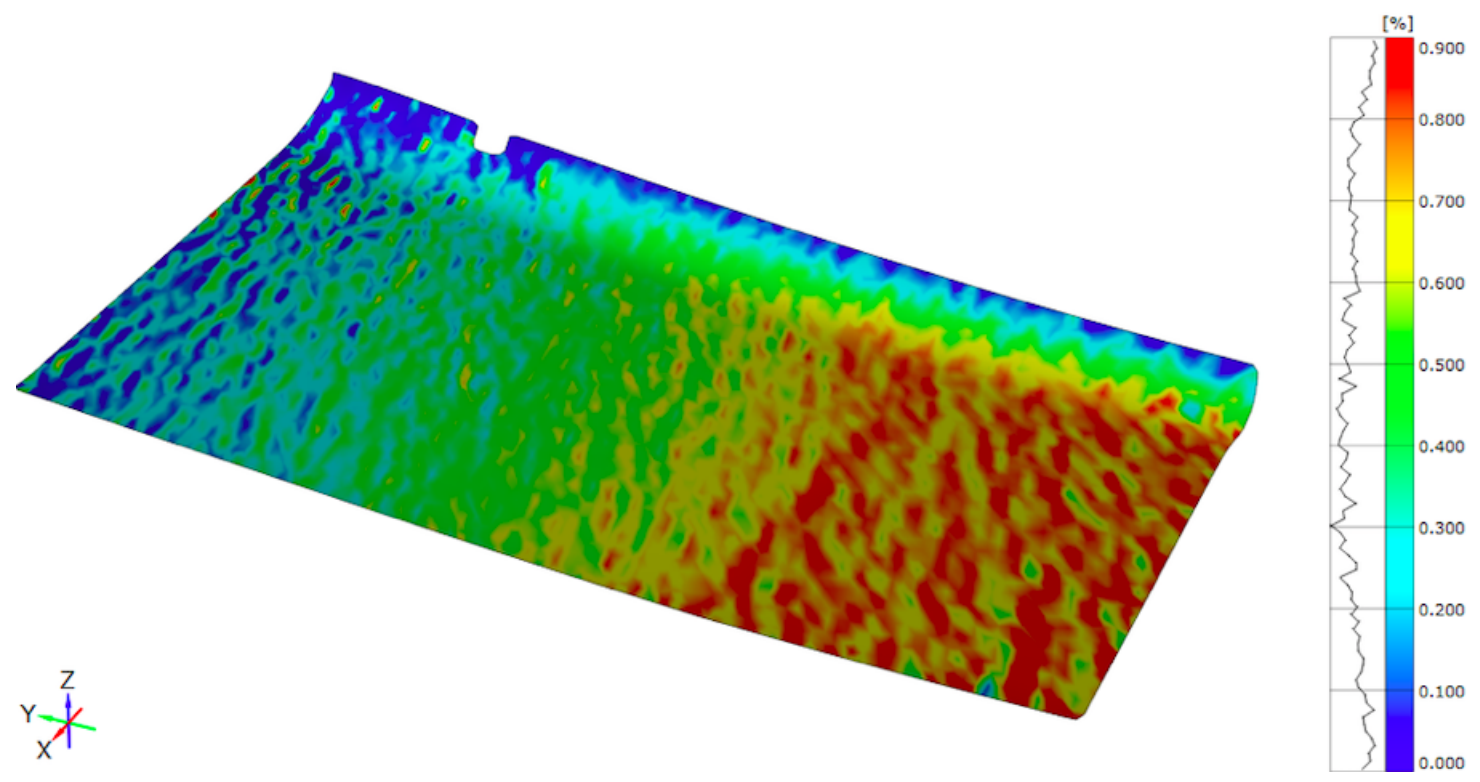

Figure 85: Four-point bending panel bottom surface axial strain at 10 psi inflation pressure

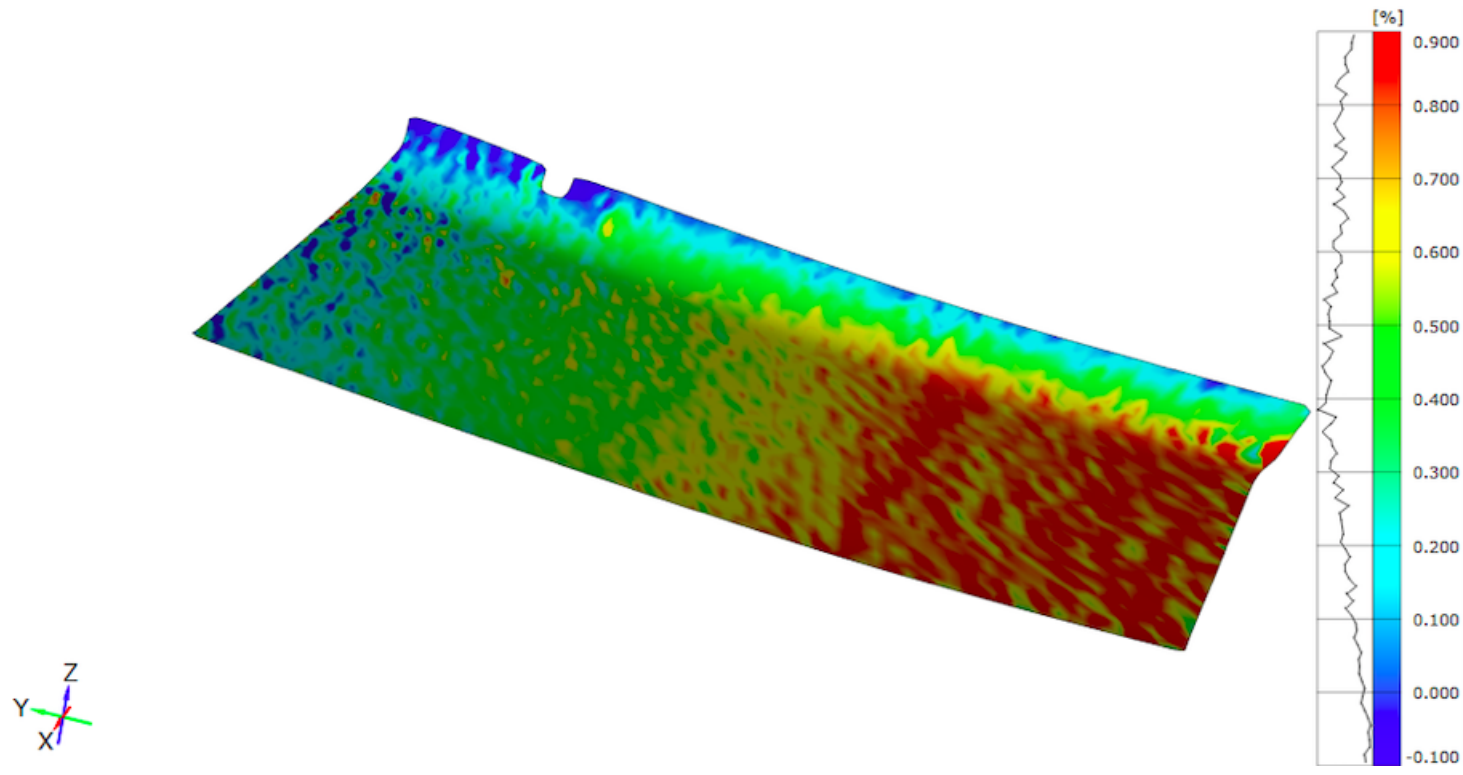

Figure 86: Four-point bending panel bottom surface axial strain at 15 psi inflation pressure

Figures 87 through 89 show the panel bottom surface transverse strain field at the point that the load frame cross head reached it max deflection at 5,10 and 15 psi inflation pressures. The transverse strain fields produced like the top surface 
were extremely nonuniform, and again observed at every inflation pressure.

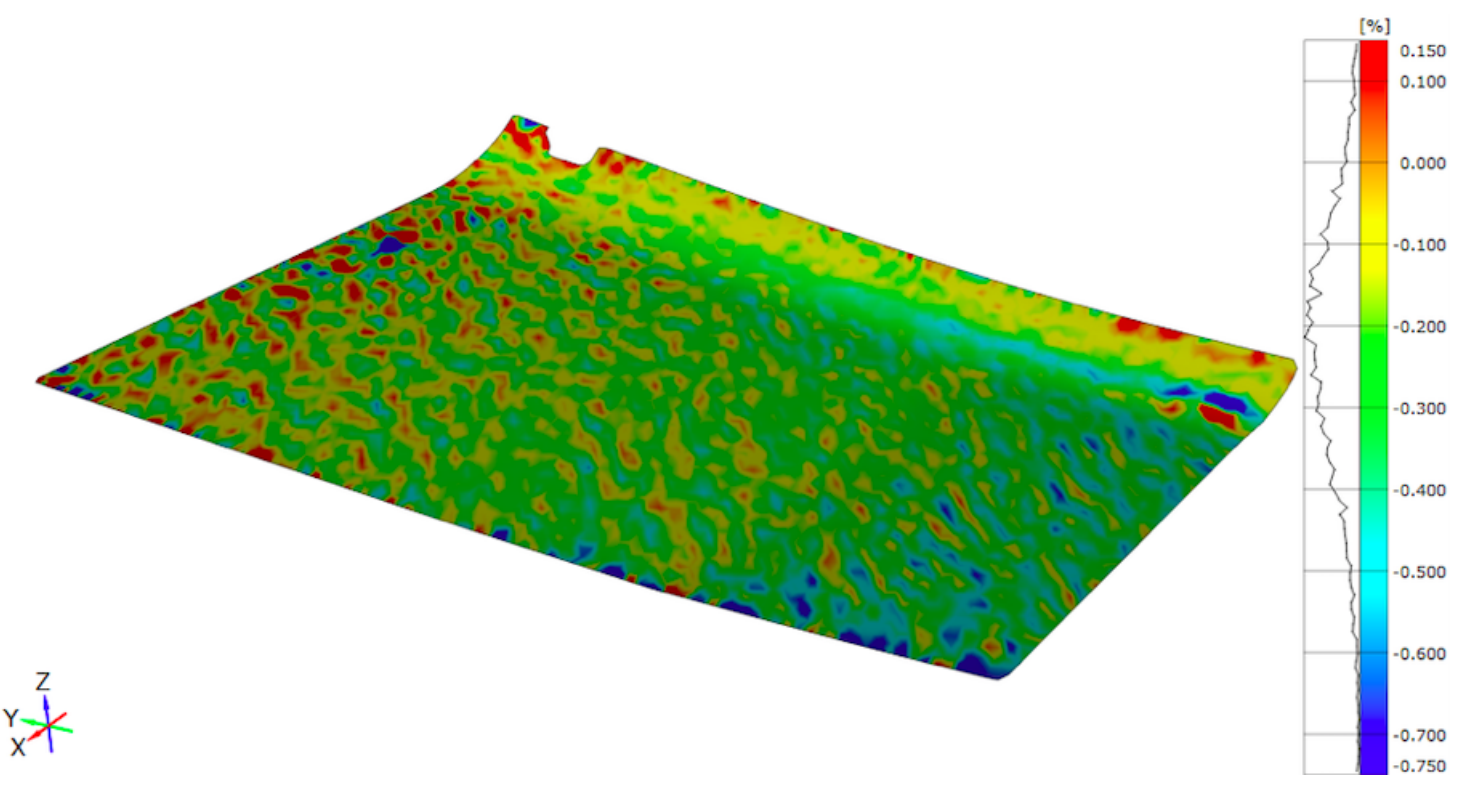

Figure 87: Four-point bending panel bottom surface transverse strain at 5 psi inflation pressure

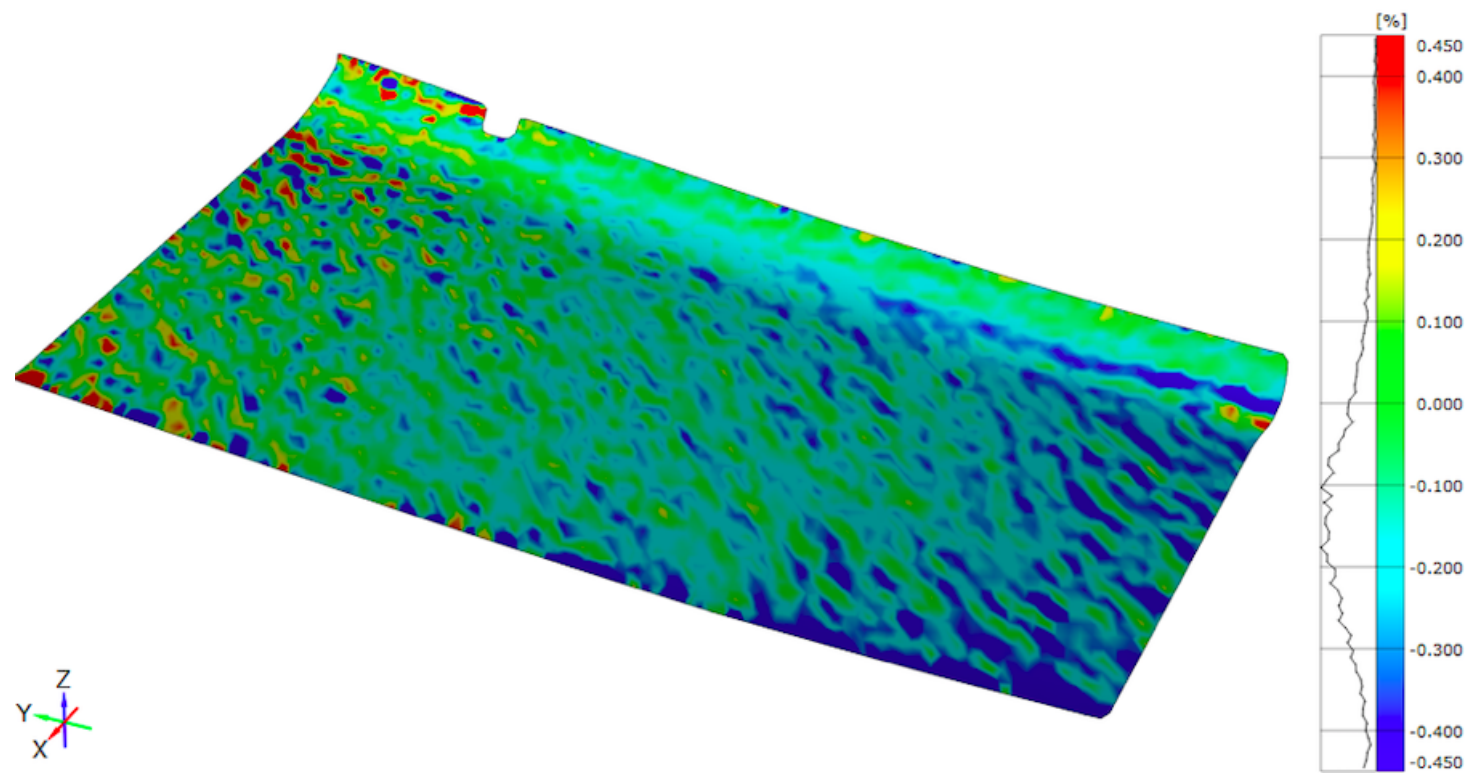

Figure 88: Four-point bending panel bottom surface transverse strain at 10 psi inflation pressure 


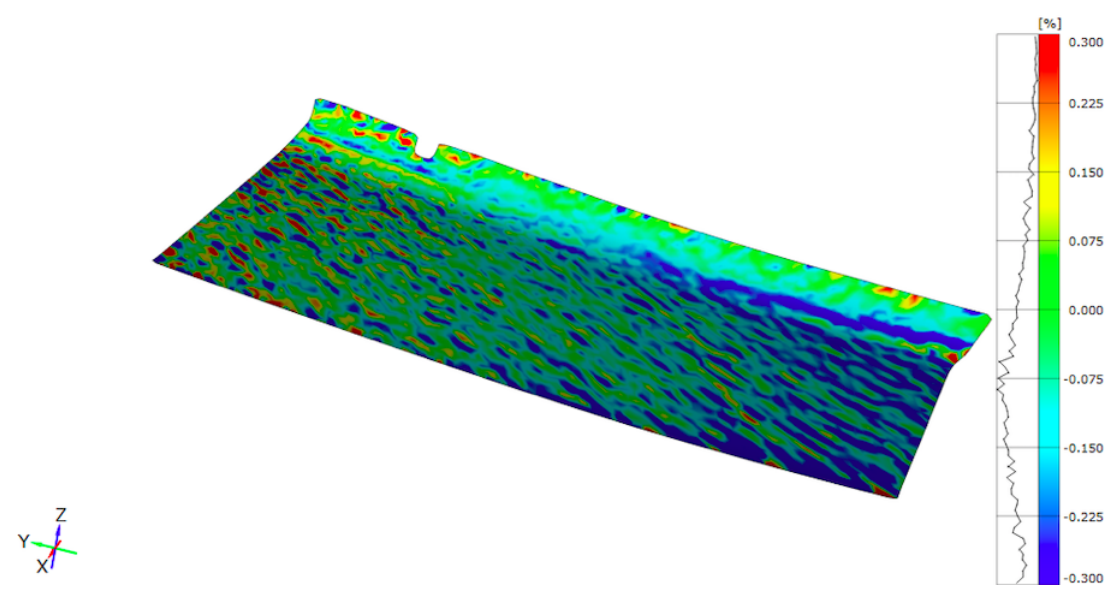

Figure 89: Four-point bending panel bottom surface transverse strain at 15 psi inflation pressure

Knowing the applied loads at the maximum three inch deflection, equation 24 can be used with the loading configuration dimensions given in figure 36 to solve for an effective axial elastic modulus under four-point bending. Table 10 gives the calculated modulus values at the tested inflation pressures.

Table 10: Experimentally determined effective modulus from four-point bending

\begin{tabular}{c|c} 
Inflation Pressure (psi) & Effective Axial Modulus (psi) \\
\hline $\mathbf{5}$ & 17,793 \\
$\mathbf{1 0}$ & 22,146 \\
$\mathbf{1 5}$ & 24,825
\end{tabular}




\section{CHAPTER 5}

\section{Conclusion}

\subsection{Conclusions}

The results presented in this study illustrate that the material properties of the drop-stitch inflatable panel skin are more complicated than traditional structural materials. As a result, predicting the behavior of drop-stitch inflatable panels proves to be difficult. This study included material characterization under a variety of load conditions to provide data to aid in improved understanding of the mechanical response of these materials.

Uniaxial and biaxial tensile tests were successful in generating stress-strain responses of the panel skin. The uniaxial testing provided estimates of Poisson's ratio in the warp and weft directions and in-plane shear modulus parallel to the warp and weft directions. The biaxial testing provided estimates of elastic moduli of the panel skin in the warp and weft directions. To compute these moduli from the biaxial results assumed Poisson ratio values obtained from uniaxial results. Comparison of these moduli with apparent skin moduli from inflated panel bend tests revealed that values obtained from biaxial tests underestimate apparent moduli from the inflated panel bend test. These differences are likely due to the effect that tensioned drop-stitches have during panel inflation which appear to increase the in-plane panel skin stiffness. The inflation experiment measurements of axial and transverse strain profiles, however, demonstrate significant nonuniform strains associated with tension of the drop-stitches when the panel is inflated.

The inflation testing was also successful in generating estimates of the orthotropic elastic response of the panel due to inflation. The measured skin stiff-

nesses due to inflation were relatively high as compared to those obtained from tensile and biaxial tests. It's expected that values obtained during inflation exper- 
iments provide a better representation of in-service load conditions experienced by drop-stitch inflatable drop-stitch panels.

The four-point bend tests characterized the displacement and strain fields of the panel top and bottom surfaces during panel bending. Correlation of these data with classical beam theory provided an estimate of the effective Young's modulus of the skin material. These apparent moduli values were found to be higher than those determined by uniaxial, biaxial and inflation experiments. It was also observed that while the panel exhibited typical beam bending behavior when analyzing axial strains on the top and bottoms surfaces, the transverse strains were found to be highly nonuniform.

The experimentally determined material properties determined throughout this research are summarized in figure 90 below.

\begin{tabular}{|c|c|c|c|c|c|c|}
\hline \multicolumn{7}{|c|}{ Summary of Experimentally Determined Material Properties } \\
\hline Experiment & $\begin{array}{c}\text { Inflation } \\
\text { Pressure (psi) }\end{array}$ & $\mathrm{E}_{1}(\mathrm{psi})$ & $\mathrm{E}_{2}(\mathrm{psi})$ & $v_{12}$ & $\mathrm{~V}_{21}$ & $\mathrm{G}_{12}(\mathrm{psi})$ \\
\hline $\begin{array}{c}\text { Uniaxial } \\
\text { Tensile Testing }\end{array}$ & - & 20,451 & 9,770 & 0.4211 & 0.7873 & 1,885 \\
\hline \multirow{4}{*}{$\begin{array}{c}\text { Biaxial Tensile } \\
\text { Testing }\end{array}$} & 5 & 7,823 & 8,603 & - & - & - \\
\cline { 2 - 7 } & 10 & 9,591 & 10,141 & - & - & - \\
\hline \multirow{3}{*}{$\begin{array}{c}\text { Inflation } \\
\text { Testing }\end{array}$} & 15 & 10,816 & 11,395 & - & - & - \\
\cline { 2 - 7 } & 10 & 10,863 & 21,572 & - & - & - \\
\hline \multirow{2}{*}{$\begin{array}{c}\text { Four-Point } \\
\text { Bend Testing } \\
\text { (CBT) }\end{array}$} & 15 & 11,207 & 21,752 & - & - & - \\
\cline { 2 - 7 } & 10 & 11,349 & 21,743 & - & - & - \\
\hline
\end{tabular}

Figure 90: Summarized material properties

In conclusion, this research met it's objective of characterizing the constituent material properties and mechanical response of inflatable drop-stitch panels subjected to uniaxial, biaxial, inflation, and panel bending load conditions using a new drop-stitch material system and three dimensional digital image correlation analyses. 


\subsection{Recommendations for future research}

Modeling drop-stitch inflatable structures is a very complex topic due to their unique nature. In addition to providing characterizations not seen before in inflatable drop-stitch structure research utilizing three-dimensional digital image correlation, this research also highlighted areas of focus for future work.

Further research into four-point bending experiments is recommended. Specifically, research into the nonuniform transverse strain fields found on the panel surfaces due to four-point loading could aid in development of improved bending models. In addition, further research into determining the contribution of shear deformation in panel bending is recommended. Also, future research into determining the shear modulus of the panel skin using a shearing test fixture is recommended. Lastly, another topic for future research would be to determine the effect of drop-stitch yarns on inflation induced skin stresses and whether tensioned drop yarns introduce 3-dimensional effects that are not considered by thin-walled pressure vessel theory. 


\section{LIST OF REFERENCES}

[1] P. V. Cavallaro and A. M. Sadegh, "Air-inflated fabric structures," Naval Undersea Warfare Center Division Newport, United States, Tech. Rep., 2006.

[2] Ž. Penava, D. Šimić-Penava, and Ž. Knezic, "Determination of the elastic constants of plain woven fabrics by a tensile test in various directions," Fibres \&3 Textiles in Eastern Europe, 2014.

[3] "Drop-stitch inflatable boat ramp," Navatek, accessed on 3-22-2020. [Online]. Available: https://www.navatekltd.com/drop-stitch.html

[4] R. Pearlman. "Inside sierra nevada's inflatable space habitat for astronauts in lunar orbit." 2019. [Online]. Available: https://www.space.com/ sierra-nevada-inflatable-habitat-moon-gateway.html

[5] P. V. Cavallaro, A. M. Smith, Sadegh, and C. J. Quigley, "Bending behavior of plain-woven fabric air beams: Fluid-structure interaction approach," Naval Undersea Warfare Center Division Newport, United States, Tech. Rep., 2006.

[6] A. Alich, "Modeling, simulation and investigation of inflatable drop-stitch panels with finite element analysis," Master's thesis, University of Rhode Island (URI), 2019.

[7] J. e. a. Górszczyk, "Application of digital image correlation (dic) method for road material testing," Materials (Basel, vol. 12, no. 15, pp. 1-19, 2019.

[8] "Standard test method for in-plane shear response of polymer matrix composite materials by tensile test of a $45^{\circ}$ laminate," Agencies of Department of Defense, Tech. Rep., 2001.

[9] L. R. DiGiovanna, "Characterizing the mechanical properties of drop stitch inflatable structures," Massachusetts Institute of Technology, Tech. Rep., 2013.

[10] P. V. Cavallaro and R. W. Smith, "Conceptual inflatable fabric structures for protective crew quarters systems in space vehicles and space habitat structures," Naval Undersea Warfare Center Division Newport, United States, Tech. Rep., 2015.

[11] P. V. Cavallaro, C. J. Hart, and A. M. Sadegh, "Mechanics of air-inflated drop-stitch fabric panels subject to bending loads," in ASME 2013 International Mechanical Engineering Congress and Exposition. American Society of Mechanical Engineers, 2013, pp. V009T10A055-V009T10A055.

[12] A. P. Boresi and R. J. Schmidt, Advanced Mechanics of Materials: Sixth Edition. John Wiley and Sons, Inc., 2003. 
[13] P. V. Cavallaro, "Technology mechanics overview of air-inflated fabric structures," Naval Undersea Warfare Center Division Newport, United States, Tech. Rep., 2006.

[14] R. Felicissimo, "Exploration of the mechanical properties of both ridged and inflated drop stitch fabric material," Master's thesis, City University of New York (CUNY), 2015.

[15] J. Falls and J. Waters, "Bending tests of inflatable dropstitch panels," in 11th International Conference on Fast Sea Transportation, FAST, 2011.

[16] M. Smith, "Mechanical response of polymer-fabric skin materials used in inflatable drop-stitch structures," Master's thesis, University of Rhode Island (URI), 2019.

[17] A. Nettles, "Basic mechanics of laminated composite plates," National Aeronautics and Space Administration, Tech. Rep., 1994.

[18] W. Rosen, "A simple procedure for experimental determination of the longitudinal shear modulus of unidirectional composites," Material Sciences Corporation, Tech. Rep., 1972.

[19] D. Roylance, "Beam displacements," Massachusetts Institute of Technology, Tech. Rep., 2000. 
APPENDIX A

MATLAB script: Inflation stresses

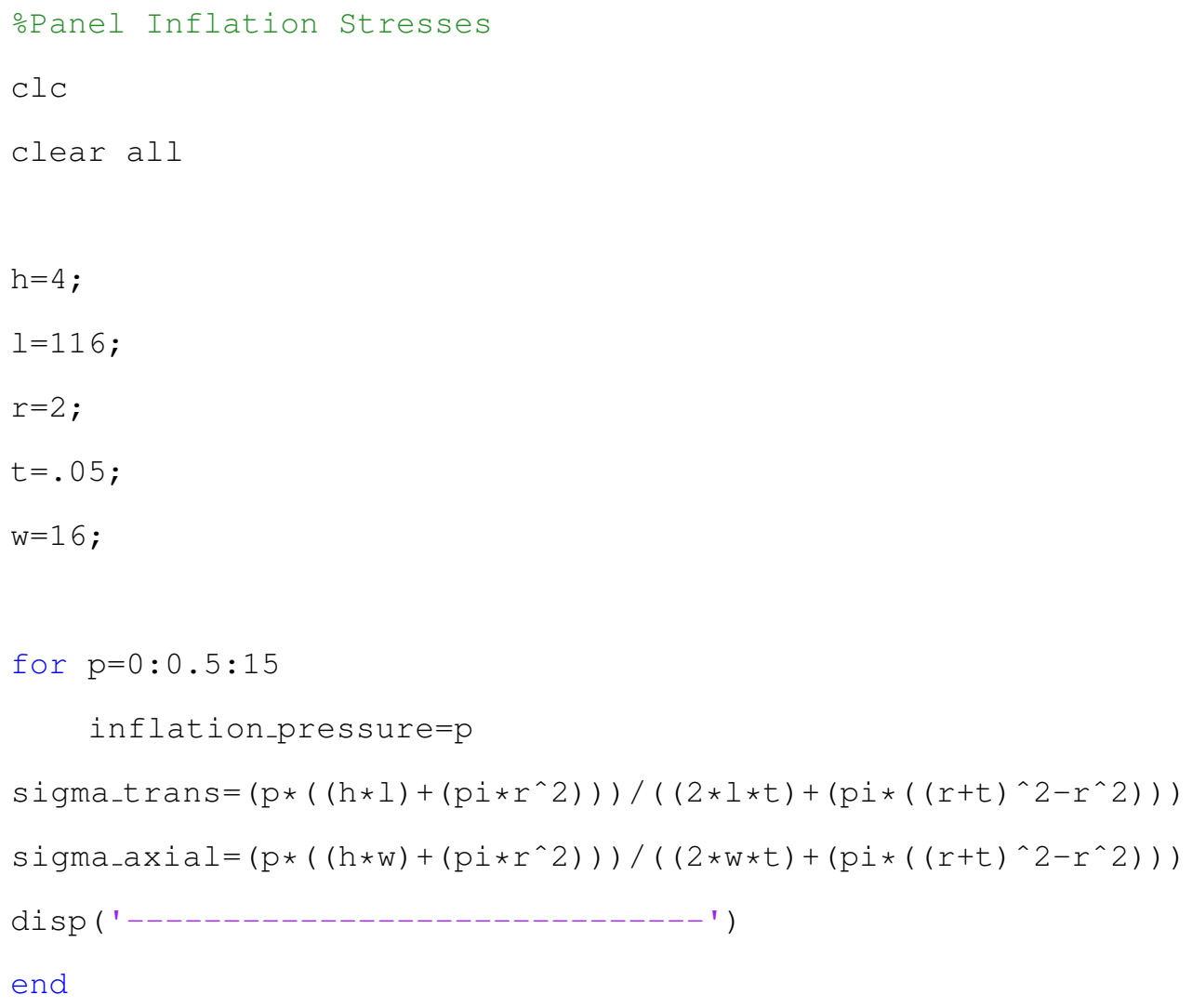




\section{APPENDIX B}

\section{MATLAB script: Effective modulus CBT}

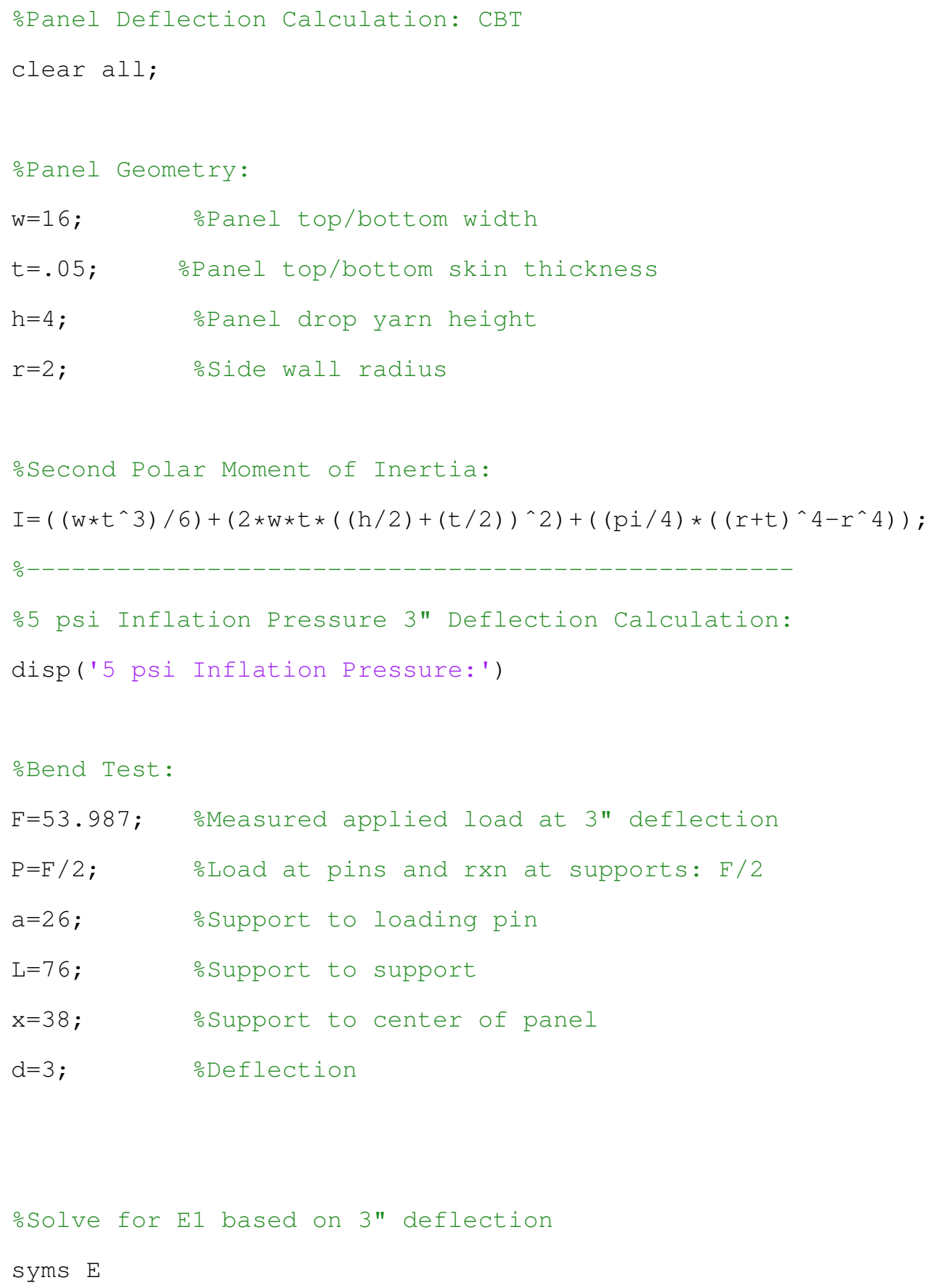




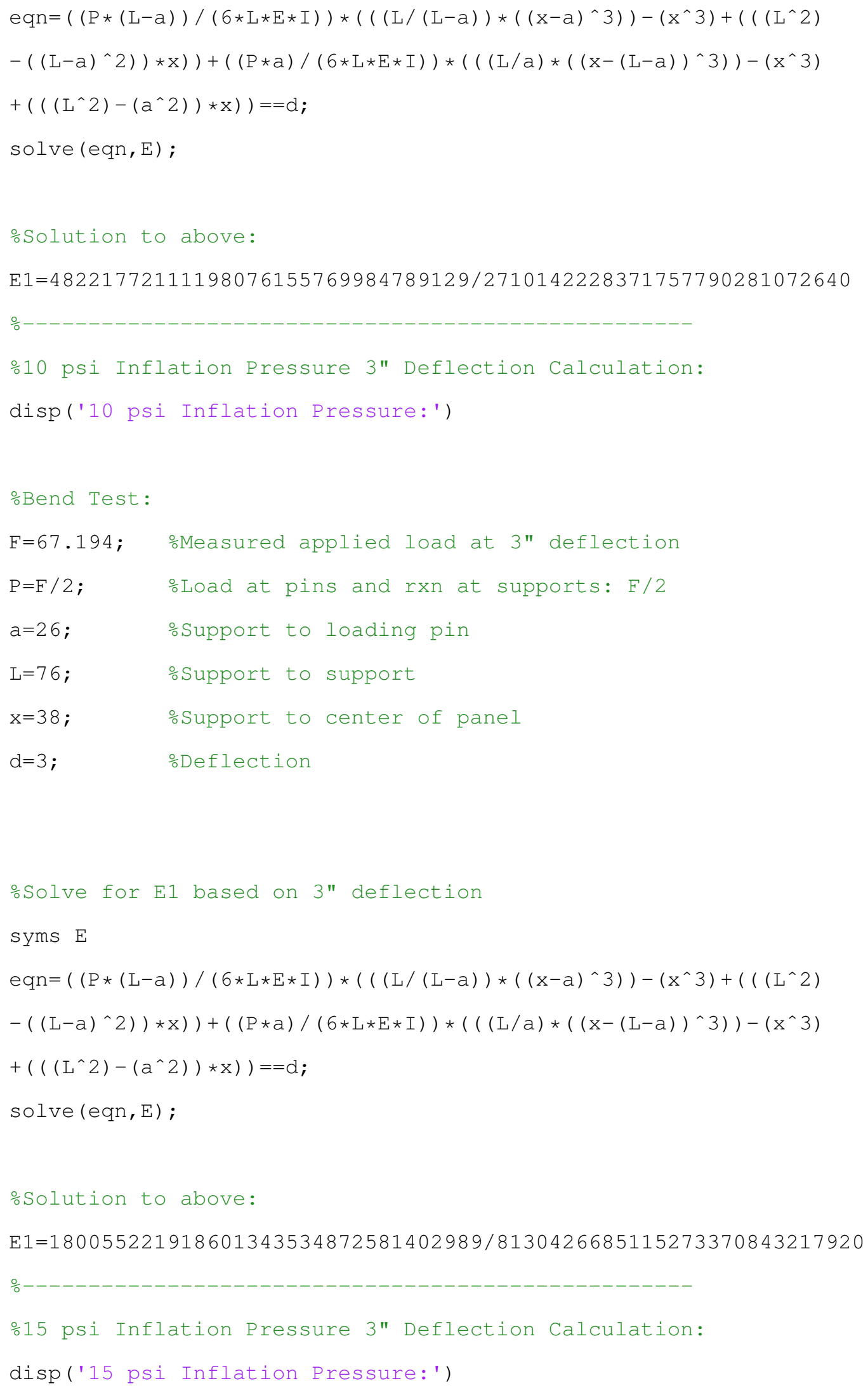


$\circ$ Bend Test:

F=75.323; $\quad$ Measured applied load at 3" deflection

$\mathrm{P}=\mathrm{F} / 2$; $\quad \frac{\text { LLod }}{2} \quad \mathrm{t}$ ins and rxn at supports: $\mathrm{F} / 2$

$\mathrm{a}=26$; $\quad$ osupport to loading pin

$\mathrm{L}=76$; $\quad$ Support to support

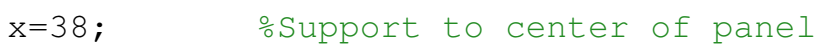

$\mathrm{d}=3 ; \quad \quad \frac{\circ}{2} \quad$

Solve for E1 based on 3" deflection

syms E

$\operatorname{eqn}=((P \star(L-a)) /(6 * L \star E \star I)) *\left(((L /(L-a)) *((x-a) \wedge 3))-\left(x^{\wedge} 3\right)+\left(\left(\left(L^{\wedge} 2\right)\right.\right.\right.$

$\left.\left.\left.-\left((L-a)^{\wedge} 2\right)\right) * x\right)\right)+((P \star a) /(6 * L * E \star I)) *\left(((L / a) *((x-(L-a)) \wedge 3))-\left(x^{\wedge} 3\right)\right.$

$\left.+\left(\left(\left(\mathrm{L}^{\wedge} 2\right)-\left(\mathrm{a}^{\wedge} 2\right)\right) * \mathrm{x}\right)\right)==\mathrm{d}$;

solve (eqn, E);

ㅇolution to above:

$E 1=201837953992541124637544970099703 / 8130426685115273370843217920$ 


\section{BIBLIOGRAPHY}

"Standard test method for in-plane shear response of polymer matrix composite materials by tensile test of a $45^{\circ}$ laminate," Agencies of Department of Defense, Tech. Rep., 2001.

Alich, A., "Modeling, simulation and investigation of inflatable drop-stitch panels with finite element analysis," Master's thesis, University of Rhode Island (URI), 2019.

Boresi, A. P. and Schmidt, R. J., Advanced Mechanics of Materials: Sixth Edition. John Wiley and Sons, Inc., 2003.

Cavallaro, P. V., "Technology mechanics overview of air-inflated fabric structures," Naval Undersea Warfare Center Division Newport, United States, Tech. Rep., 2006.

Cavallaro, P. V., Hart, C. J., and Sadegh, A. M., "Mechanics of air-inflated dropstitch fabric panels subject to bending loads," in ASME 2013 International Mechanical Engineering Congress and Exposition. American Society of Mechanical Engineers, 2013, pp. V009T10A055-V009T10A055.

Cavallaro, P. V. and Sadegh, A. M., "Air-inflated fabric structures," Naval Undersea Warfare Center Division Newport, United States, Tech. Rep., 2006.

Cavallaro, P. V., Smith, Sadegh, A. M., and Quigley, C. J., "Bending behavior of plain-woven fabric air beams: Fluid-structure interaction approach," Naval Undersea Warfare Center Division Newport, United States, Tech. Rep., 2006.

Cavallaro, P. V. and Smith, R. W., "Conceptual inflatable fabric structures for protective crew quarters systems in space vehicles and space habitat structures," Naval Undersea Warfare Center Division Newport, United States, Tech. Rep., 2015.

DiGiovanna, L. R., "Characterizing the mechanical properties of drop stitch inflatable structures," Massachusetts Institute of Technology, Tech. Rep., 2013.

Falls, J. and Waters, J., "Bending tests of inflatable dropstitch panels," in 11th International Conference on Fast Sea Transportation, FAST, 2011.

Felicissimo, R., "Exploration of the mechanical properties of both ridged and inflated drop stitch fabric material," Master's thesis, City University of New York (CUNY), 2015.

Górszczyk, J. e. a., "Application of digital image correlation (dic) method for road material testing," Materials (Basel, vol. 12, no. 15, pp. 1-19, 2019. 
"Drop-stitch inflatable boat ramp," Navatek, accessed on 3-22-2020. [Online]. Available: https://www.navatekltd.com/drop-stitch.html

Nettles, A., "Basic mechanics of laminated composite plates," National Aeronautics and Space Administration, Tech. Rep., 1994.

Pearlman, R. "Inside sierra nevada's inflatable space habitat for astronauts in lunar orbit." 2019. [Online]. Available: https://www.space.com/ sierra-nevada-inflatable-habitat-moon-gateway.html

Penava, Ž., Šimić-Penava, D., and Knezic, Ž., "Determination of the elastic constants of plain woven fabrics by a tensile test in various directions," Fibres $\mathscr{E}$ Textiles in Eastern Europe, 2014.

Rosen, W., "A simple procedure for experimental determination of the longitudinal shear modulus of unidirectional composites," Material Sciences Corporation, Tech. Rep., 1972.

Roylance, D., "Beam displacements," Massachusetts Institute of Technology, Tech. Rep., 2000.

Smith, M., "Mechanical response of polymer-fabric skin materials used in inflatable drop-stitch structures," Master's thesis, University of Rhode Island (URI), 2019. 